DOC. NO. NARF-62-15T FZK-9-180

\title{
ENERGY STORAGE MECHANISMS IN LIQUID HYDROGEN MASTEK
}

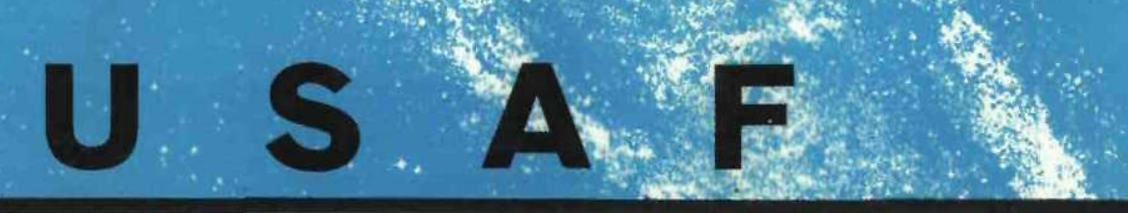

\section{NUCLEAR AEROSPACE RESEARCH FACILITY}

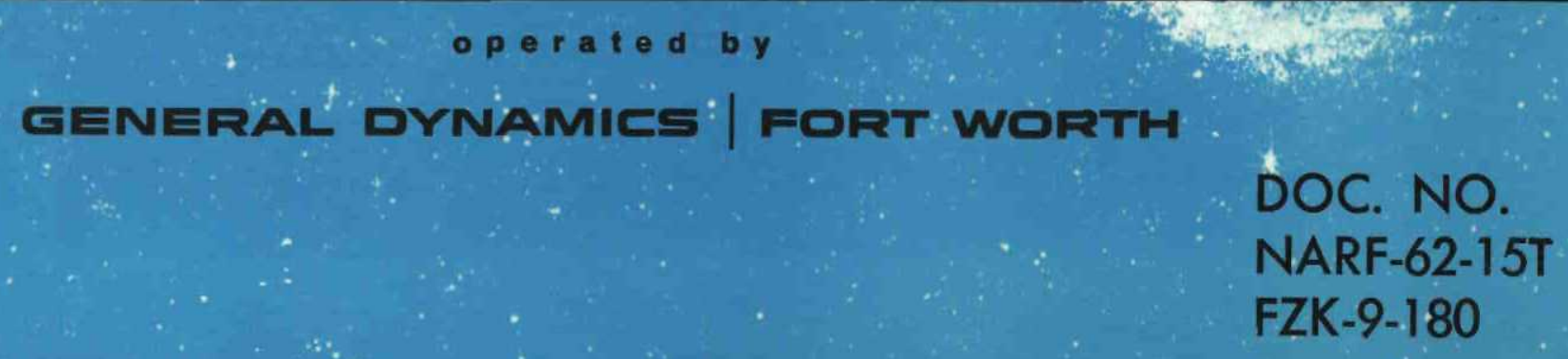




\section{DISCLAIMER}

This report was prepared as an account of work sponsored by an agency of the United States Government. Neither the United States Government nor any agency Thereof, nor any of their employees, makes any warranty, express or implied, or assumes any legal liability or responsibility for the accuracy, completeness, or usefulness of any information, apparatus, product, or process disclosed, or represents that its use would not infringe privately owned rights. Reference herein to any specific commercial product, process, or service by trade name, trademark, manufacturer, or otherwise does not necessarily constitute or imply its endorsement, recommendation, or favoring by the United States Government or any agency thereof. The views and opinions of authors expressed herein do not necessarily state or reflect those of the United States Government or any agency thereof. 


\section{DISCLAIMER}

Portions of this document may be illegible in electronic image products. Images are produced from the best available original document. 
DOC. NO.

NARF-62-15T

FZK-9-180

\section{U S A F}

\section{NUCLEAR AEROSPACE RESEARCH FACILITY}

31 OCTOBER 1962

\section{ENERGY STORAGE MECHANISMS IN LIQUID HYDROGEN}

H. G. CARTER

SECTION II, TASK 1, ITEM 5

OF FZM-2386

CONTRACT

AF 33(657)-7201

ISSUED BY THE

ENGINEERING 


\begin{abstract}
Energy-storing molecular products of radiation in liquid hydrogen are examined from the point of view that the explusion of potential energy in the form of such products may lead to lower design weights for nuclear rockets. It is found that a significant fraction of the energy deposited by radiation in Ilquid hydrogen is stored temporarliy through the dissociation and excitation of $\mathrm{H}_{2}$ molecules. Experimental and theoretical results are consistent with a rate of dissipation of such energy into thermal motion which is much slower than the rate at which the radiation products are expelled from the tank. It is shown that, if this is the case, relatively high levels of infrared radiation are to be expected but that such energy will not rapidiy be translated into thermal motion. It is concluded that, insofar as radiation heating of the propellant is a significant design factor, further consideration should be given to radiation-induced reactions in 11quid bydrogen and that any radiation-heating experiments should be interpreted in terms of possible energy-storage effects.
\end{abstract}




\section{Blank page}




\section{REPORT SUMMARY}

Hydrogen-propelled nuclear vehicles play a leading role in presently envisaged space missions. Therefore, the processes associated with radiation-heating in liquid hydrogen are worthy of attention. Of particular interest from the standpoint of propellantshield design is the formation of energy-storing molecular species through which the production of actual thermal energy is temporarily postponed. Consideration of potential energy expelled from the system in the form of such specles may lead to lower overall design weights, owing to less stringent provisions for shielding, pressurew vessel construction, and/or boll-off。 The object of the present study is to determine whether an appreciable amount of the energy deposited by radiation is stored in radiation products and whether such products survive in the liquid long enough to be expelled from the system. In realistic cases most of the radiation products are formed near the exit, and the time required for their expulsion is only about 20 seconds.

Energy deposited in liquid hydrogen by neutral radiation is initially transferred to recoll protons or Compton-scattered electrons in the case of neutrons or gamma rays, respectively. When such charged particle pass through the hydrogen, which is normally almost entirely in the $\mathrm{H}_{2}$ ground state, approximately equal amounts of energy go into the production of H-atom pairs, 
through the formation of the repulsive ${ }^{3} \Sigma_{\mathrm{u}} \mathrm{H}_{2}$ state and into the production of $\mathrm{H}_{2}^{+}$1ons. By means of reactions such as

$$
\mathrm{H}_{2}^{+}+\mathrm{H}_{2} \Longrightarrow \mathrm{H}_{3}^{+}+\mathrm{H}: \mathrm{H}_{3}^{+}+\mathrm{e}^{-} \Longrightarrow \mathrm{H}_{2}^{*}+\mathrm{H}
$$

the ionization process leads indirectly to further dissociation and excltation. Through ionic and dissociative reactions alone, about $43 \%$ of the energy deposited is temporarily stored in the production of $\mathrm{H}$ atoms and excited $\mathrm{H}_{2}$ molecules. Most of the remaining energy is inftially released by the ejection of $H$ atoms from repulsive molecular configurations. The total amount of energy storage could be substantially higher than 43\%, depending upon the efficiency of energetic $\mathrm{H}$ atoms in producing excited $\mathrm{H}_{2}$ states. Energy storage through para- to ortho-hydrogen conversion is found to be insignificant because of the low excitation energy involved.

Whether the time required for the dissipation of stored energy into thermal motion is less or greater than the 20 seconds or so spent in the system by 1rradlated molecules depends on the rate at Which radiation products return to the $\mathrm{H}_{2}$ ground state. This rate is determined by (1) the rate of recombination of $\mathrm{H}$ atoms, (2) the rate of de-excitation of $\mathrm{H}_{2}$ molecules and (3) the rate of transmission to the tank walls of Infrared rays from molecular trans1tions induced in the liquid. 
It is probable that at liquid-hydrogen temperatures the recombination of $\mathrm{H}$-atom pairs proceeds by a 3-body reaction with an $\mathrm{H}$ atom as the third body. Analysis of theoretical and experimental results shows that the efficiency of 3-atom recombination is certainly low enough to permit the expulsion of a significant fraction of $\mathrm{H}$ atoms at $\mathrm{H}$-atom densities corresponding to moderate radiation levels and, furthermore, that the experimental data are consistent with an efficiency low enough to permit significant H-atom expulsion at arbitrarily high radiation levels.

De-excitation may occur through the superelastic scattering process, in which a molecule in an excited state interacts with one or more unexcited molecules and gives up its excitation energy as recoll kinetic energy. A recent wave-mechanical treatment shows that the cross section for this reaction falls off drastically at low collision energies. Assuming that the energy dependence revealed by the theoretical results can be extended to energies corresponding to liquid-hydrogen temperatures and that only isolated binary collisions are important, it is found that the mean lifetime in the liquid against superelastic de-excitation of the first vibrational level of $\mathrm{H}_{2}$ is of the order 100 minutes. The assumption of isolated binary collisions on which the estimate is based appears to be theoretically sound in the case of liquid hydrogen. Confirmation is needed of the assumed temperature dependence of the de-excitation rate for the first level, and the calculations should be extended to include higher levels and H-atom recombination. 
The probabilities of infrared-ray emission and absorption by $\mathrm{H}_{2}$ molecules are related to the half-widths of experimentally observed absorption peaks. Recent spectral measurements provide a means of calculating the probabilities for some of the fundamental transitions. It is found that the lifetime against emission in the liquid is of the order of seconds but that the mean free path against absorption by the formation of the original type of $\mathrm{H}_{2}$ state is so short that infrared rays are not rapidly transmitted to the walls. If energy storage mechanisms are important, it should be possible to observe experimentally the flux of infrared rays trapped in the irradiated liquid.

It is concluded that a significant fraction of radiation energy is temporarily stored in liquid hydrogen. While information on the rate at which stored energy is dissipated into thermal motion is not conclusive, what evidence exists is at least consistent with a rate of dissipation which is much slower than the rate at which radiation products are expelled from the system. Therefore, insofar as radiation heating of the propellant has a significant influence on the design of a nuclear-powered vehicle, further calculations of the rates of radiation-induced molecular reactions are warranted; and any experimental data obtained on the radiation heating of liquid hydrogen should be interpreted in the light of possible delays in the equipartition of energy stored in radiation products. 
TABLE OF CONTENTS

Page

ABSTRACT

REPORT SUMMARY

LIST OF FIGURES 11

LIST OF TABLES 13

I. INTRODUCTION 15

II. RADIATION-ENERGY DEPOSITION 19

2.1 Radiation-Induced Reactions in Hydrogen 19

2.2 Production of Potential and Kinetic Energy by
Means of Ionic and Dissoclative Reactions

2.3 Production of Potential Energy Through Inelastic
$\mathrm{H}-\mathrm{H}_{2}$ Collisions

2.4 Para- to Orthohydrogen Conversion 35

III. DISSIPATION OF STORED ENERGI 41

IV. RECOMBINATION OF H ATOMS $4 ?$

4.1 Three-Body Recombination in Liquid Hydrogen it?

4.2 Experimental Results on Recombination 54

V. SUPERELASTIC DE-EXCITATION OF H2 MOLECULES 61

5.1 Superelastic Process in Ilquid Hydrogen 67

5.2 Wave-Mechanical Treatment of Inelastic and Superelastic Scattering Between $\mathrm{H}_{2}$ Molecules

5.3 Relaxation-Time of the First Vibrational $\mathrm{H}_{2}$-State in Liquid Hydrogen

5.4 Applicability of the Isolated Binary Collision Assumption for Liquid Hydrogen

5.5 Need for Superelastic Cross Sections in Prediction of Propellant Heating 
TABLE OF CONTENTS (cont'd)

Page

VI. INFRARED-RAY EMISSION AND ABSORPTION 79

6.1 Emission and Absorption Probabilities 79

6.2 Significance of Experimental Infrared Absorption
Spectra for the Liquid

6.3 Emission and Self-Absorption in Liquid Hydrogen 88

VII. CONCLUSIONS 97

APPENDIX A. CALCULATION OF H3 LIFETIME 99

APPENDIX B。 THE SLOWING DOWN OF EXCITED H2 MOLECULES 109

APPENDIX C. CALCULATION OF ELECTROMAGNETIC TRANSITION PROBABILITIES

REF ERENCES

DISTRIBUTION

NOTE

The following pages are intentionaliy left blank: $2,4,12,14,18,46,60,98,112,124,130$ : 


\section{LIST OF FIGURES}

Figure

Page

$1 \quad$ Principal Ionic and Dissoclative Events Resulting from the Irradiation of Hydrogen

2 Potential-Energy Contours for an H-Atom Approaching an $\mathrm{H}_{2}$ Molecule

$3 \mathrm{H}_{3}$ Potential- Energy Contours and Representative System-Point Trajectory in Configuration Space

4 Cross Section for the Excitation of the First Vibrational $\mathrm{H}_{2}$ Level by Inelastic Scattering Between a Pair of $\mathrm{H}_{2}$ Molecules

5 Vibrational Relaxation Time for the First $\mathrm{H}_{2}$ Level at an Unspecified Low Density

6 Infrared Absorption Spectra for Dense Hydrogen

A-I Permissible Modes of $\mathrm{H}_{3}$ Vibration 
Blank page 


\section{LIST OF TABLES}

Table

Page

I Binding Energies of Stable Hydrogen Configurations

24

II $\mathrm{H}_{3}$ Lifetimes Against Disintegration Leading to the Ground State of $\mathrm{H}_{2}$

III Emission and Absorption Coefficients for Unperturbed $\mathrm{H}_{2}$ Molecules

IV Emission and Absorption Coefficients for H2 Molecules in Liquid Hydrogen and Mean-Free-Path of Emitted Radiation 
Blank page 


\section{INTRODUCTION}

Hydrogen-propelled nuclear-powered vehicles play a leading role In presently envisaged space missions. Consequently, much consideration has been given to the gross deposition of radiation energy inside the propellant storage system. It is customarily assumed that such radiation energy is instantly translated into familiar thermal effects. It would seem, however, that the microscopic processes associated with radiation heating in liquid hydrogen are worthy of attention. of particular interest from the standpoint of propellant-shield and pressure-vessel design should be the formation of energy-storing molecuiar species through which the production of actual thermal energy is temporarily postponed. Consideration of potential energy expelied from a storage system in the form of such species could lead to lower overall design weights, owing to less stringent provisions for shielding, pressure-vessel construction, and/or bollofi.

The object of this study is to determine whether a significant amount of energy is temporarily stored through the formation of radiation products in Iiquid hydrogen and whether such products survive in the Ilquid for a long enough period of time that their mechanical expulsion competes favorably with their return to the $\mathrm{H}_{2}$ ground state through the liberation of kinetic energy. It is assumed that if radiation heating of the propeliant has a signifio cant effect on the design of a space vehicle, then the possible 
expulsion of an appreciable fraction of the deposited energy in nonthermal form is an important consideration. What advantage in design weight might accrue to the expulsion of a particular fraction of the energy in the form of radiation products is beyond the scope of the present inquiry.

That energy-storage mechanisms in liquid hydrogen should be looked into is suggested by the general nature of the propellant heating problem. In an important class of cases one has to reckon with radiation-induced heating only while the nuclear power source is in operation and while the propellant is being expelled from the storage tank at a high rate. Furthermore, in foreseeable space vehicles, the fraction of the total volume of propellant which is nearest the radiation source and which therefore absorbs most of the radiation is also nearest the storage exit or pump. Therefore, volume elements of the liquid are exposed to intense radiation only shortly before they are expelled, and, hopefully, the potential energy created may not have time to dissipate into thermal motion.

It is. expected that full-scale propellant tanks for "one way" vehicles will be completely emptied in around ten minutes. Using a typical tank length of the order of 100 feet and an effective energyflux relaxation length of three feet (which is reasonable for mixed neutron and gamma radiation in liquid hydrogen), it is seen that the majority of the molecules affected by radiation are expelled within 20 seconds after being affected. Hence, a 20-second period can be 
taken as the minimum time for which energy must remain stored in radiation products if energy-storage effects are to be significant. An attempt is made in the following sections to determine whether a significant fraction of the energy deposited by radiation In liquid hydrogen is stored as potential energy and is not dissipated into thermal energy in less than the 20-second time required for the expulsion of the radiation products. In Section II, the molecular reactions which lead to appreciable energy storage are identified, and a lower limit is set on the amount of energy which is stored at least temporar1ly. Section IIIoutlines the general mechanisms of stored-energy dissipation. Section IV examines the dissipation process in which H-atom pairs are recombined through energy transfer to a third body. Section $V$ is concerned with the rate of molecular de-excitation through the production of relative kinetic energy of recoil between molecules. Section VI considers the molecular emission and re-absorption probabilities which determine the rate of migration of infrared rays through the liquid and to the walls of the system. Lastly, Section VII is devoted to conclusions. 
Blank page 


\section{RADIATIONळENERGY DEPOSITION}

\subsection{Radiation-Induced Reactions in Hydrogen}

Energy deposited in liquid hydrogen by neutral radiation is Initially transferred to recoll protons or Comptonmscattered electrons in the case of neutrons and gamma rays, respectively. The energy imparted to such charged particles is very large compared to the binding energy which holds atoms or molecules together (see Table I). Consequently, energy deposition by energetic charged particles involves the same kind of destructive processes regardless of the charge of the energetic particle. If it is assumed that the slowing down of charged particles of different types and energies leads to the same production of ionic and molecular species per unit energy deposited, then the types and proportions of molecular species produced are independent of the composition and energy spectrum of the radiation field. Although this assumption, that the amount produced of a particular species depends only on the energy deposited, may be only roughly valid, it should provide a reasonable basis for energy-storage calculations。

A compatible assumption is that the products of $\alpha$-particle radiation in hydrogen gas, which are known from both theoretical and experimental studies, are presentative of the products formed by protons and electrons in the liquid. A 1936 paper by Eyring, Hirschfelder, and Taylor (Ref。1) presents much basic information on the ionic and dissociative reactions induced by $\alpha$-rays in 
hydrogen gas; more recent papers (Refs. 2, 3) indicate no major revisions of their conclusions. The processes described in Reference 1 do not appear to involve the mobility or density of molecules in such a way that reactions due to protons and electrons In the liquid should be much different from those due to a-rays in the gas. It is therefore assumed that the types and proportions of various radiation products are independent of the molecular density, as well as the charged-particle type and energy.

Radiation in hydrogen produces, in addition to certain relatively stable excited states of $\mathrm{H}_{2}$, the following species which do not disintegrate immediately: $\mathrm{H}, \mathrm{e}^{-}, \mathrm{H}^{+}, \mathrm{H}_{2}^{+}, \mathrm{H}_{3}^{+}, \mathrm{H}^{-}$, and $\mathrm{H}_{2}^{-}$. The primary positive ions (those which are formed directly by the passage of a charge particle) are quite predominantly of the type $\mathrm{H}_{2}^{+}$. This type of Ionization requires $15.37 \mathrm{ev}$. However, it has been found that for each such ion created the charged particle somehow loses about $33 \mathrm{ev}$ (Ref. 1). The extra energy must go into excitation and kinetic energy of recoll. Eyring et al. inferred that most of this extra energy goes into the so-called $3_{\Sigma_{u}}$ exclted electronic state of $\mathrm{H}_{2}$, the excitation energy of which is about $11.6 \mathrm{ev}$ (Ref。1; Ref.4, p. 38). The ${ }^{3} \Sigma_{\mathrm{u}}$ state is a repulsive configuration; it has no equilibrium position for the formation of an $\mathrm{H}_{2}$ molecule and therefore dissociates into two $\mathrm{H}$ atoms as soon as it is formed (Ref. 5; Ref. 6, p. 344; Ref。 7). Since the dissociation energy of the $\mathrm{H}_{2}$ molecule is $4.73 \mathrm{ev}$, the kinetic energy of each $\mathrm{H}$ atom thus formed is $(11.6-4.7) / 2$ or $3.4 \mathrm{ev}$. If 
the inference of Eyring et al. Is correct, it is seen that roughiy one pair of such $\mathrm{H}$ atoms is produced for each $\mathrm{H}_{2}^{+}$ion formed. As the concentration of radiation products is always relatively small, energetic $H$ atoms eventually go into thermal equilibrium through a series of elastic and inelastic collisions with $\mathrm{H}_{2}$ molecules.

$H$ atoms are produced by other means than $3 \Sigma_{u}$ excitation. When a newly formed $\mathrm{H}_{2}^{+}$ion comes into the vicinity of an $\mathrm{H}_{2}$ molecule which has not been excited by the radiation, the reaction

$$
\mathrm{H}_{2}^{+}+\mathrm{H}_{2} \Longrightarrow \mathrm{H}_{3}^{+}+\mathrm{H}
$$

has a high probability of occurrence. Although a certain "energy barrier" (activation energy) exists for this reaction, the height of this barrier is found to be so low that most ion-molecule pairs have enough kinetic energy to surmount it, even at liquid-hydrogen temperatures. Other possible reactions for the decomposition of $\mathrm{H}_{2}^{+}$ are

$$
\begin{aligned}
& \mathrm{H}_{2}^{+}+\mathrm{e}^{-} \Longrightarrow 2 \mathrm{H} \text { (formation of a momentary repulsive state), } \\
& \mathrm{H}_{2}^{+}+\mathrm{e}^{-}+\mathrm{H}_{2} \Longrightarrow 2 \mathrm{H}_{2}^{*}\left(\underset{\left.\mathrm{H}_{2}\right)}{3-\text { body reaction resulting in excited }}\right. \\
& \mathrm{H}_{2}^{+}+\mathrm{H}_{2} \Longrightarrow \mathrm{H}_{2}+\mathrm{H}+\mathrm{H}^{+} \text {(endothermal reaction). }
\end{aligned}
$$

The last three reactions are belleved to offer little competition to that which leads to $\mathrm{H}_{3}^{+}$. Thus, $\mathrm{H}_{2}^{+}$, the predominant ion resulting directly from radiation, is likely to enjoy only a fleeting existence before reacting with $\mathrm{H}_{2}$ to beget $\mathrm{H}_{3}^{+}$and $\mathrm{H}_{\text {。 }}$ 
The chain of events which is started by the radiation-induced ionization of the $\mathrm{H}_{2}$ molecule is terminated, as far as ions are concerned, with the neutralization of the $\mathrm{H}_{3}^{+}$ion. $\mathrm{H}_{3}^{+}$readily combines with an electron in a 2-body reaction, since the energy of formation may be absorbed in the excitation and kinetic energy of the reaction products. When $\mathrm{H}_{3}^{+}$is neutralized, the resulting $\mathrm{H}_{3}$ configuration has an equal probability of being in either of two repulsive states. In one of these states, two of the $H$ atoms bind together and repel the third atom; in the other state, all three of the atoms are mutually repulsive. Thus, the neutralization of $\mathrm{H}_{3}^{+}$ could be expressed as

$$
\mathrm{H}_{3}^{+}+\mathrm{e}^{-} \Longrightarrow \frac{1}{2}\left(\mathrm{H}_{2}+\mathrm{H}\right)+\frac{1}{2}(3 \mathrm{H}) \text {. }
$$

(Free and lightly bound electrons should be avallable from the Ionization of $\mathrm{H}_{2}$ 。)

The energy yields from the combination of $\mathrm{H}_{2}^{+}$and $\mathrm{H}_{2}$ and from the neutralization of $\mathrm{H}_{3}^{+}$can be calculated from the binding energies

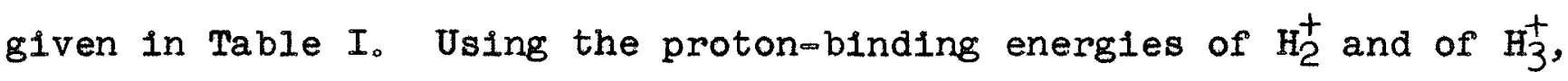
the energy yield of the reaction

$$
\mathrm{H}_{2}^{+}+\mathrm{H}_{2} \Longrightarrow \mathrm{H}_{3}^{+}+\mathrm{H}
$$

is $(2.74-2.64)=.10 \mathrm{ev}$. (Since the proton-binding energy of $\mathrm{H}_{3}^{+}$ is only known approximately, this result could easily be in error, and the reaction might even be slightly endothermal; the calculation serves to show that the energy yield, if any, is small.) The yield 
of the reaction

$$
\mathrm{H}_{3}^{+}+\mathrm{e}^{-} \Longrightarrow \mathrm{H}_{2}^{*}+\mathrm{H}
$$

is $(13.54-2.74)=10.8 \mathrm{ev}$, where the ionization energy of the $\mathrm{H}$ atom $(13.54 \mathrm{ev})$ has been used. When an $\mathrm{H}_{2}$ molecule is formed by this process, it is apt to be in an excited state, as is indicated by the asterisk. The reaction

$$
\mathrm{H}_{3}^{+}+\mathrm{e}^{-} \longrightarrow 3 \mathrm{H}
$$

yields $(13.54-2.74-4.73)=6.07 \mathrm{ev}$, where use has been made of the $\mathrm{H}_{2}$ dissociation energy $(4.73 \mathrm{ev})$.

2.2 Production of Potential and Kinetic Energy by Means of Ionic and Dissociative Reactions

The energy yields of the most important ionic and dissociative reactions are given in Section 2.1, but the form in which the energy appears is a different matter. It was established that in the ${ }^{3} \Sigma_{u}$ reaction two $\mathrm{H}$ atoms are formed, each with a kinetic energy of $3.4 \mathrm{ev}$. The yield of the reaction between $\mathrm{H}_{2}^{+}$and $\mathrm{H}_{2}$ (Eq。2-2) is small enough that the form of the energy is unimportant for this discussion. The energy yield from the $\mathrm{H}_{3}^{+}$neutralization which results in $3 \mathrm{H}$ (Eq. 2-4) must all be in the form of kinetic energy, since the energy avaliable is less than the lowest excited electronic level of the $\mathrm{H}$ atom $(10.2 \mathrm{ev})$. In this latter reaction, if the triatomic hydrogen molecule formed in the neutralization is linear and symmetrically repulsive, as seems likely (Ref's。11, 12), then the kinetic energy is equally divided between two $H$ atoms, each of them having $3.02 \mathrm{ev}$. 
Table I

Binding Energies of Stable Hydrogen Configurations

\begin{tabular}{|c|c|c|}
\hline $\begin{array}{l}\text { Configuration and } \\
\text { Type of Binding }\end{array}$ & Binding Energy & Source \\
\hline $\mathrm{H}_{2}: \mathrm{H} \Longleftrightarrow \mathrm{H}$ & $4.73 \mathrm{ev}$ & (Ref。8, p. 567) \\
\hline $\mathrm{H}_{2}: \mathrm{H}_{2}^{+} \longleftrightarrow \mathrm{e}^{-}$ & $15.37 \mathrm{ev}$ & (Ref。1) \\
\hline $\mathrm{H}: \mathrm{H}^{+} \longleftrightarrow \mathrm{e}^{-}$ & $13.54 \mathrm{ev}$ & (Ref。9, p. 234) \\
\hline $\mathrm{H}_{2}^{+}: \mathrm{H} \Longleftrightarrow \mathrm{H}^{+}$ & $2.64 \mathrm{ev}$ & $\left(\begin{array}{l}\text { Ref } 7 ; ; \\
\text { p. } 354)\end{array}\right.$ \\
\hline $\mathrm{H}_{3}^{+}: \mathrm{H}_{2} \Longleftrightarrow \mathrm{H}^{+}$ & $2.74 \mathrm{ev}$ & (Rer. 10) \\
\hline
\end{tabular}

In the case where neutralization of $\mathrm{H}_{3}$ results in an $\mathrm{H}_{2}$ molecule and an $\mathrm{H}$ atom ( $\mathrm{Eq}, 2-3$ ), the unstable $\mathrm{H}_{3}$ molecule is again linear but the repulsion is not symmetric. Two of the $H$ atoms unite and repel the third. The resulting $\mathrm{H}_{2}$ molecule is presumably formed in one of the vibrational states of the ' $\Sigma_{g}$ (lowest) orbital state. The reason for this inference is that the energy of the $\mathrm{H}_{3}$ state which dissociates to $\mathrm{H}_{2}+\mathrm{H}$ is lower than that which dissociates to 3H (Ref.1, p.486). Therefore, the orbitals involved should correspond to three $\mathrm{H}$ atoms in their lowest electronic states, two of which vibrate with an energy which is not large enough to overcome their mutual binding. Of course, this is a supposition based on an assumed analogy between the two $\mathrm{H}_{3}$ states and does not rigorously rule out the possibilities (1) that the H atom is formed in its first excited electronic state with the consequence that $10.2 \mathrm{ev}$ of the 10.8-ev yield is given up in the form of Lyman 
radiation or (2) that the $\mathrm{H}_{2}$ molecule is inftially formed in the $B^{\prime} \Sigma_{g}$ orbital state (Ref。5), afterwards going to a vibrational state of ${ }^{B} \Sigma_{g}$ by transition. (The latter process is possible only if the 10.8-ev calculated yleld is slightly in error, since the lowest $B^{\prime} \Sigma_{g}$ level is at $11.14 \mathrm{ev}$ )

Assuming that a molecule in a ${ }^{\prime} \Sigma_{g}$ state (ground electronic state) results from the reaction ( $E q, 2-3$ ), it is most probable that the state is one of high vibrational excitation with an energy approaching that of dissociation. That one of the many closely spaced upper levels will be excited is suggested by a molecular temperature model of the highly excited $\mathrm{H}_{3}$ complex. The vibrational $\mathrm{H}_{3}$ modes involve maximum-energy-level spacings of the same order as those in the $\mathrm{H}_{2}$ molecule, $1 . \mathrm{e}_{0}$, about $0.5 \mathrm{ev}$. The $10.8 \mathrm{-ev}$ excitation produced in the neutralization of $\mathrm{H}_{3}^{+}$is so high that a classical equipartition of energy should occur among the degrees of freedom of the triatomic molecule. Therefore, the probability that the $\mathrm{H}_{2}$ molecule is formed in a low-lying level should be smal1. Evidence which lends support to this point of view is given in Section 2.3 and in Appendix $A$, where it is shown on a quantummechanical basis that for an energetic $\mathrm{H}_{3}$ complex modes of decomposition leading to low-lying $H_{2}$ levels are improbable. Thus, the vibration energy of the $\mathrm{H}_{2}$ molecule formed in the reaction (Eq。2-3) should be of the order of $4.7 \mathrm{ev}$, in which case the total kinetic energy evolved is approximately $(10.8-4.7)=6.1 \mathrm{ev}$. Then, from conservation of momentum, the kinetic energies of the atom and molecule are $4.1 \mathrm{ev}$ and $2.0 \mathrm{ev}$, respectively。 
What might be called the princlpal events with respect to ionic and dissociative reactions are summarized in Figure 1. The more obscure reactions are omitted, and the assumption of exactly one ${ }^{3} \Sigma_{\mathrm{u}}$ excitation for each $\mathrm{H}_{2}$ ionization is merely a convenient approximation. The two major branches represent reactions which are presumed to proceed concurrently (not as optional branches) after the deposition of $33 \mathrm{ev}$ by energetic charged particles. Figure 1 shows that five $\mathrm{H}$ atoms are formed for every $33 \mathrm{ev}$. deposited. Since the potential energy between pairs of $H$ atoms is $4.73 \mathrm{ev}$, the energy stored in the production of $\mathrm{H}$ atoms is $(1 / 2)(5)(4.73)=11.8 \mathrm{ev}$, or $36 \%$ of the energy deposited. If it is assumed that the energy of the excited $\mathrm{H}_{2}$ state is of the order of $4.7 \mathrm{ev}$, then the energy stored through 1onic and dissociative reactions alone comes to $43 \%$. Because of the effective flimsiness of atoms and molecules, the deposition of energy by primary charged particles and by energetic secondary lons does not lead directly to a large amount of molecular translational energy along the path of ionization (Ref.1; Ref。9, p. 336). Therefore, most of the remaining 57\% of deposited energy is initially released in the disintegration of repulsive molecular configurations, going mainly into the motion of energetic $H$ atoms. Figure $I$ shows the infital Hatom energies. 


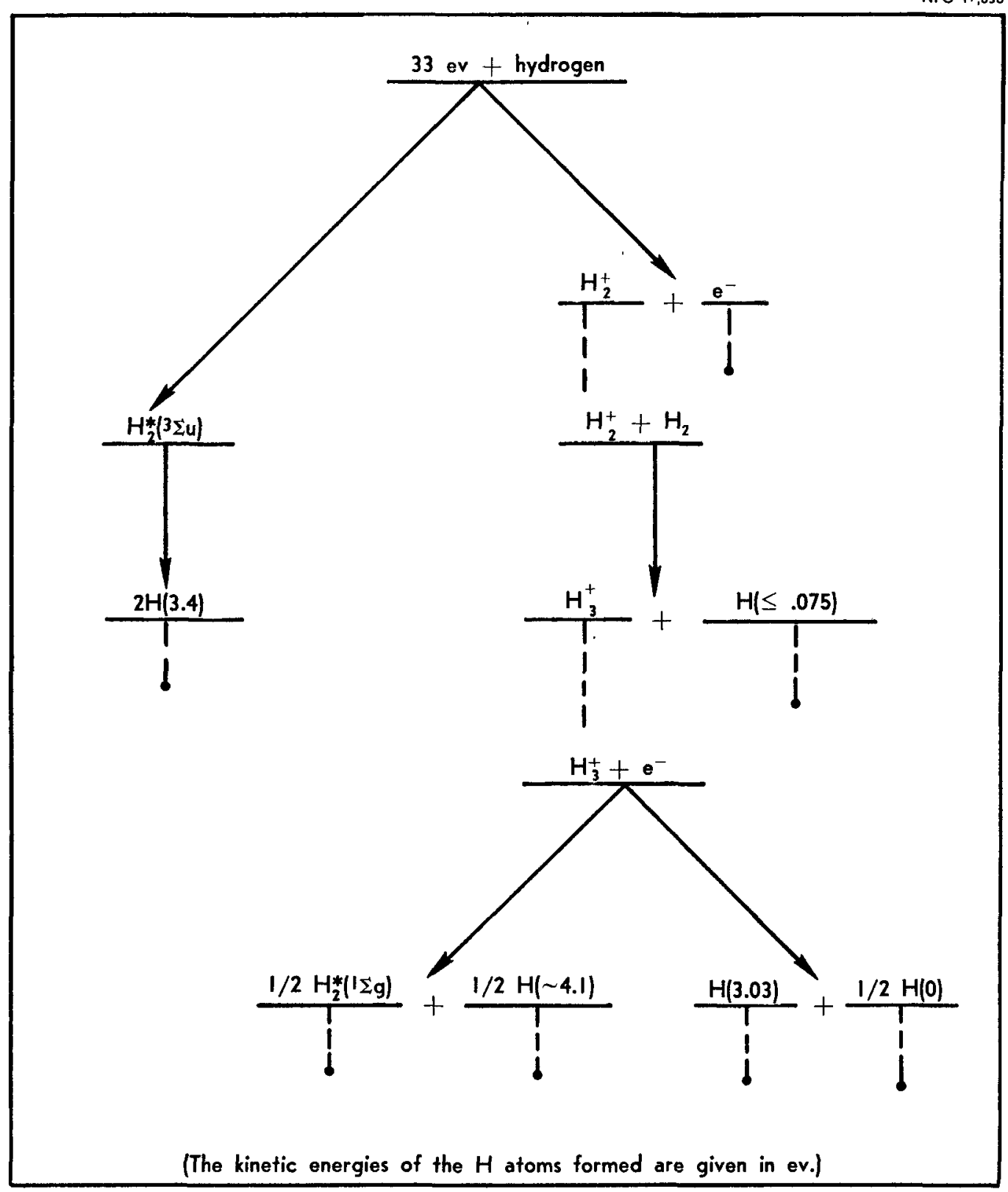

Figure 1. Principal Ionic and Dissociative Events 
2.3 Production of Potential Energy Through Inelastic $\mathrm{H}-\mathrm{H}_{2}$ Collisions Considerably more than $43 \%$ of the deposited energy w1ll be stored if collisions between energetic $\mathrm{H}$ atoms and $\mathrm{H}_{2}$ molecules are effective at producing excited $\mathrm{H}_{2}$ states. The permissible energy levels for an $\mathrm{H}_{2}$ molecule excited to low states of vibration and rotation (in the ground electronic state) are described by (Ref.13)

$$
E_{J V}=0.015(I / 2) J(J+1)+0.533 v,
$$

where $E_{J v}$ is the excltation energy in ev of a molecule in its Jth rotational state and its vth vibrational state. It is seen that excitation of the first vibrational or the first rotational level takes up $0.533 \mathrm{ev}$ or $0.015 \mathrm{ev}$, respectively (in the centre-of-mass system). Vibrational excitation is accompanied by rotational excitation in general but may be thought of as occurring independently, since the two types of motion are only slightly coupled.

Since energetic $\mathrm{H}$ atoms are ejected with energies on the order of $3 \mathrm{ev}$, it is possible for this kinetic energy to be stored by both rotational and vibrational excitation (excited electronic states are inaccessible because of insufficient energy). Whether the amount of energy stored in this way compares favorably with that which is dissipated in the recoll of $\mathrm{H}_{2}$ molecules depends on the cross section for momentum transfer between the atom and molecule, as well as on the excitation cross section. Although realistic momentum-transfer cross sections might possibly be obtalned from thermodynamical data, 
the only available and theoretical cross sections for the inelastic process (Ref。14) are based upon rough quantum-mechanical approximations and have not been confirmed by experiment. Hence, only a general argument for a significant amount of excitation is justified. The present discussion shall only be concerned with vibrational excitation and, in Section 2.4, with a special case of rotational excitation.

The vibrational excitation of an $\mathrm{H}_{2}$ molecule by an energetic $\mathrm{H}$ atom occurs through the formation and decomposition of an $\mathrm{H}_{2}-\mathrm{H}$ (or $\mathrm{H}_{3}$ ) complex. This triatomic complex is linear - 1.e., all three atoms lie on a line (Ref. 12) - and, of course, unstable. The probability that an $\mathrm{H}_{2}$ molecule will be excited to a particular vibrational level in a collision with an $\mathrm{H}$ atom depends on (1) the probability that an $\mathrm{H}_{2}-\mathrm{H}$ complex will be formed and (2) the probability that the mode of decomposition corresponds to the particular vibrational level of $\mathrm{H}_{2}$.

Whether or not a complex is formed is determined by the effective potential-energy field presented by the molecule and by the energy and direction of the incoming $\mathrm{H}$ atom. Figure 2 shows a contour map of the theoretical potential field in an ordinary 2dimensional coordinate system. The calculations were made by Hirschfelder et al. (Refs。11, 12) and are in close agreement with calculations made recently by a more refined method (Ref。15). In order for a complex to be formed, the $\mathrm{H}$ atom must, in general, cut across a potential "ridge" and enter one of the "basins" at 


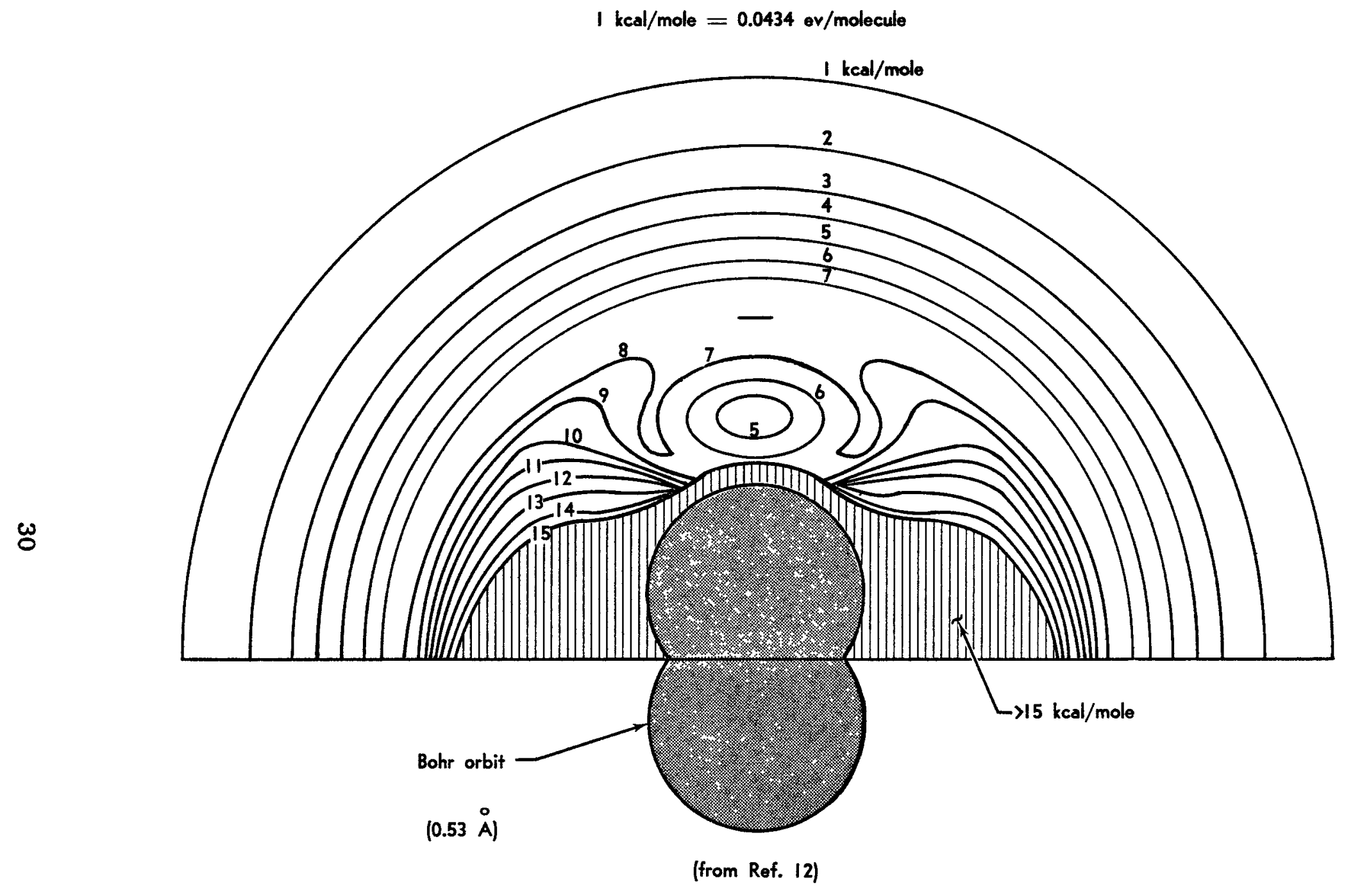

Figure 2. Potential-Energy Contours for an $\mathbf{H}$ Atom Approaching an $\mathrm{H}_{2}$ Molecule 
either end of the molecule. Viewing the collision in the centerof-mass system (where atom and molecule approach each other with equal momenta), it is clear that, in collisions which are solid enough to effect an appreclable momentum transfer, there should be a high probability of complex formation in all but the most unfavorable configurations.

The motion of the entire system before and after the formation of a complex can best be described by a potential-energy contour map in configuration space. Figure 3 is a configuration space map of the potential shown in Figure 2. Each possible set of positions for the three $\mathrm{H}$ atoms is represented by a single point in configuration space, and the movement of such a point along the surface corresponds to the movements of all three atoms along various degrees of freedom. The situation is in direct analogy to a frictionless point mass moving on an uneven surface in a gravitational fleld. A system point which has enough inftial kinetic energy to cross the potential barrier and enter the "valley" which corresponds to an $\mathrm{H}_{3}$ complex may follow a long wandering trajectory before finding its way out again. In reality, this means that the avaliable energy is suffio cient to disintegrate the complex but that this does not occur until the energy happens to become concentrated in the right degrees of internal freedom. A long lifetime against disintegration allows equipartition of energy among the various internal degrees of freedom and, hence, a high probability that the complex will disintegrate with the $\mathrm{H}_{2}$ molecule in an excited state. 
I $\mathrm{kcal} / \mathrm{mole}=0.0434 \mathrm{ev} / \mathrm{molecule}$

$$
H_{0} \rightarrow r_{b b} \rightarrow H_{b} \rightarrow r_{b c} \rightarrow H_{c}
$$

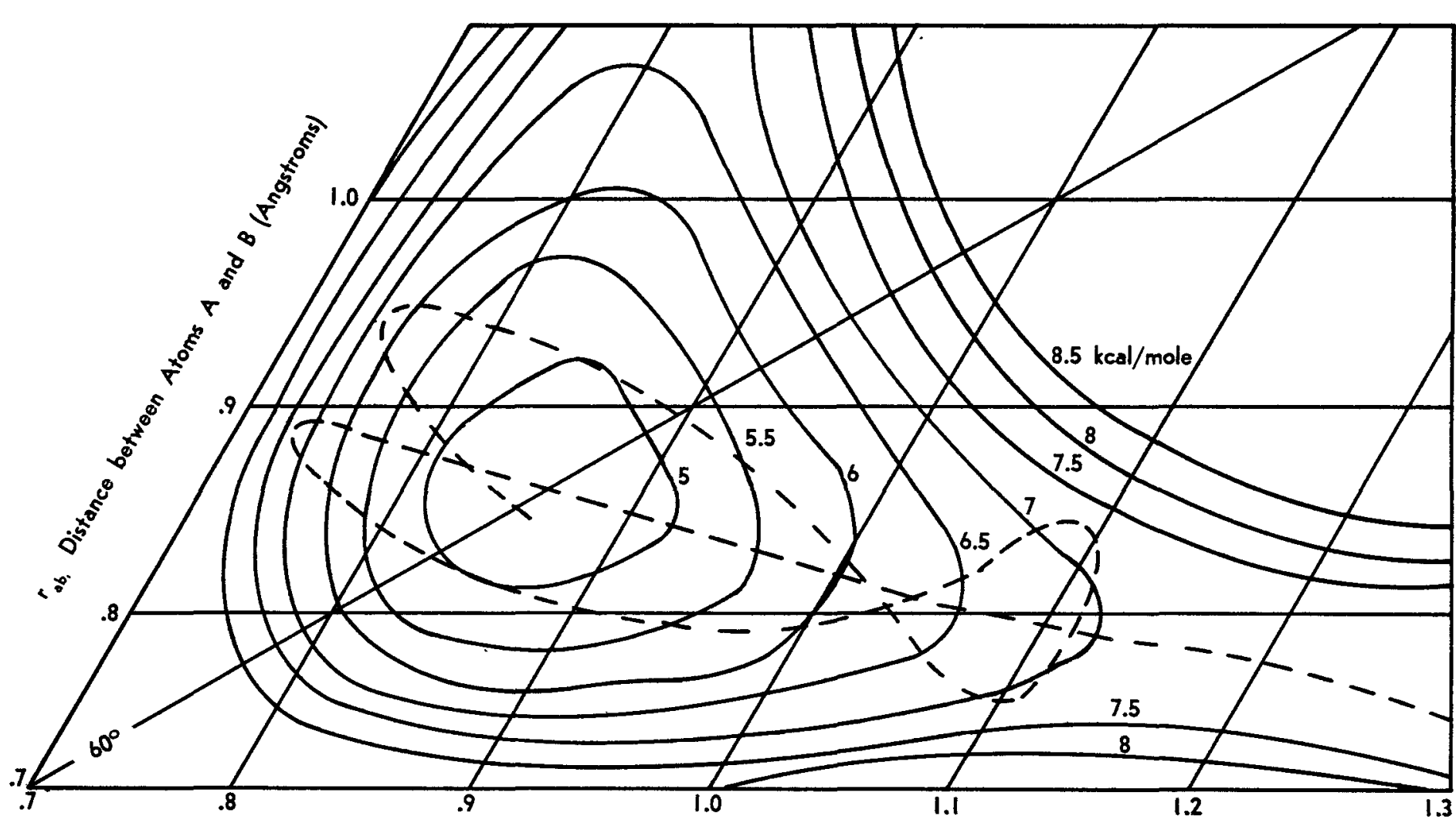

$r_{b c}=$ Distance between Atoms B and C (angstroms)

$$
\text { (from Ref. 12) }
$$

Figure 3. $\mathrm{H}_{3}$ Potential-Energy Contours and Representative System-Point Trajectory in Configuration Space 
It is found that high-energy complexes, such as those formed by $\mathrm{H}$ atoms with energies in the ev range, have extremely long lifetimes (for unstable complexes). An intultive explanation of the increase of lifetime with complex energy has been given in terms of the configuration space model by Hirschfelder and Wigner (Ref。16)。 They point out that, in general, part of the kinetic energy of the system point goes into oscillations normal to the line of least potential and that in energetic complexes this leads to a "baffing" which makes the escape of the system point difficult. A quantum. mechanical analysis of the complex lifetime shows the quantitative effect of such a baffing.

Some $\mathrm{H}_{3}$ lifetimes have been calculated by the author (Appendix A) using an adaptation of a perturbation method applied by Rosen (Ref。17) to the $\mathrm{HO}_{2}$ complex. Table II gives the Iifetimes of various $\mathrm{H}_{3}$ modes of vibration (corresponding to various domains in configuration space) against decomposition into an $\mathrm{H}$ atom and an $\mathrm{H}_{2}$ molecule in its ground state. The numbers $m$ and $p$ characterize the initial motion and refer to the degree of vibration between adjacent pairs of atoms in the complex. Because of the long lifetimes of energetic complexes, internal transitions (changes of $\mathrm{m}$ and p) are much more probable than decomposition. Hence, it is likely that when $\mathrm{H}_{3}$ disintegrates, the energy initially borne by the H atom has been well "mixed" among varlous degrees of freedom and that the $\mathrm{H}_{2}$ molecule is left in an excited state。 
Table II

$\mathrm{H}_{3}$ Iifetimes Against Disintegration Leading to the Ground State of $\mathrm{H}_{2}$

\begin{tabular}{|c|c|c|}
\hline $\begin{array}{c}E \text { (ev) } \\
\text { (Complex-Energy) }\end{array}$ & $\begin{array}{c}\mathrm{m}, \mathrm{p} \\
\left(\mathrm{H}_{3} \text { Mode) }\right.\end{array}$ & $\begin{array}{c}\tau \text { (sec }) \\
\text { (Iifetime) }\end{array}$ \\
\hline 0.5 & 6,30 & $1.0(-6)$ \\
0.5 & 4,28 & $1.7(-9)$ \\
0.5 & 3,26 & $2.2(-11)$ \\
0.5 & 1,20 & $3.6(-15)$ \\
1.0 & 4,26 & $1.2(-9)$ \\
1.0 & 3,23 & $1.5(-11)$ \\
2.0 & 7,26 & $6.5(-6)$ \\
2.0 & 5,22 & $1.6(-8)$ \\
3.0 & 10,25 & $3.3(-3)$ \\
3.0 & 8,22 & $4.7(-5)$ \\
4.0 & 13,23 & $2.8(-1)$ \\
4.0 & 12,21 & $5.2(-2)$ \\
\hline
\end{tabular}

From the preceding considerations, it appears that $H$ atoms (1) should be able to brute-force their way into the formation of an $\mathrm{H}_{3}$-complex in a large fraction of solid collisions and (2) should produce long-lived $\mathrm{H}_{3}$ states which have a good probability of leaving an excited $\mathrm{H}_{2}$ molecule upon disintegration. Thus, even though the situation is complicated by such effects as elastic collisions, rotational excitations, and collisions between the 
long-lived translating complex and molecules in the liquid, it can be assumed that an appreciable amount of energy storage occurs through the inelastic scattering of $\mathrm{H}$ atoms.

2.4 Para- to Ortho-hydrogen Conversion

In the present study the only type of rotational excitation which will be considered is the special case of para- to orthohydrogen conversion. This type of energy storage would at first seem promising, since it is an important effect in the heating of cold hydrogen gas to ordinary temperatures and since the state of higher energy (orthohydrogen) is known to have an extremely long Iifetime in the Iiquid (Ref。18, p。290). However, consideration of the mechanisms by which orthohydrogen is formed shows that radiation-induced conversion is unimportant in systems that do not permit a temperature rise of more than a few degrees.

The $\mathrm{H}_{2}$ molecule may occur in either of two "modifications". In an orthohydrogen molecule the proton spins are parallel; in a parahydrogen molecule the proton spins are antiparallel. Although either type of molecule may assume a number of excited states, orthohydrogen molecules are restricted to odd rotation numbers $\left(1, e_{0}\right.$, odd multiples of the angular momentum $\hbar$ ), whereas parahydrogen molecules are restricted to even rotation numbers. Thus, the "ground-state" rotation numbers for para- and ortho-hydrogen, respectively, are $J=0$ and $J=I$. Equation $2-5$ shows that the energy difference between the lowest states of para- and orthohydrogen is $0.015 \mathrm{ev}$. 
Orthohydrogen represents a triplet quantum-mechanical state, whereas parahydrogen represents a singlet state. It can be shown that in a re-arrangement in which there is no spin correlation, all four of the states have an equal probability of formation (Ref. 19, p. 400). Thus, in the absence of circumstances which lead to spin correlation (such as insufficient energy for a triplet state) three out of four re-arrangements lead to orthodhydrogen. Therefore, at high temperatures, hydrogen gas is a mixture of three-fourths orthohydrogen and one-fourth parahydrogen. In I1quid hydrogen, which corresponds to a thermal enerfy of only $0.0017 \mathrm{ev}$, practically all of the molecules eventually go to the lowest parahydrogen state.

Aside from drastic changes in the vibrational or electronic state of the molecule, conversion from one modification to the other results from either (1) magnetic interactions between weak magnetic dipoles, or (2) formation and subsequent decomposition of $\mathrm{H}_{3}$ complexes. Owing to parallel proton-spin alignments, orthohydrogen molecules exhibit a slight paramagnetic behavior; ortho- to parahydrogen conversion can occur through the reaction

$$
\mathrm{H}_{2}^{\circ}+\mathrm{H}_{2}^{\circ} \equiv \mathrm{H}_{2}^{\circ}+\mathrm{H}_{2}^{\mathrm{p}}
$$

with a kinet1c-energy release of $0.015 \mathrm{ev.} \mathrm{However,} \mathrm{this} \mathrm{is} \mathrm{a} \mathrm{low-}$ probability process at all temperatures (Ref. I). The reverse reaction, para- to ortho-hydrogen conversion, cannot occur by magnetic interaction between parahydrogen molecules, even when the 
energy is available. The reason for this is that parahydrogen molecules have no magnetic moments, owing to the antiparallel proton spins (in either modification the electron spins are required by the exclusion principle to be antiparaliel). The conversion of gas from "normal" (3/4 ortho-) hydrogen to parahydrogen when cooled from high temperatures (and vice versa in the reverse process) is due almost entirely to a small concentration of $\mathrm{H}$ atoms.

Conversion by complex formation may occur when an $\mathrm{H}$ atom of $\mathrm{a}$ particular proton-spin orientation occupies the potential "basin" (Fig. 2) of an $\mathrm{H}_{2}$ molecule. The molecule formed in the subsequent decomposition is a new modification if the dispossessed $H$ atom is the one opposite the incident $\mathrm{H}$ atom in the $\mathrm{H}_{3}$ complex and has the opposite proton-spin orientation. A conversion of this sort can be represented by a system point which moves into one end of the potential "valley" (Fig. 3) and emerges from the other end. The kinetic energy of the system point changes by $0.015 \mathrm{ev}$ (although the corresponding potential difference between the ends of the valley is too small to show in the 11lustration).

It is seen from Figures 2 and 3 that a complex can be formed only if sufficient kinetic energy is avallable to overcome an "activation barrier" for the reaction. Figure 2 shows that for the most favorable direction of approach the "height of this barrier, with respect to "ground" (infinite atomolecule separation), is about $7 \mathrm{kcal} / \mathrm{mole}$, or $0.30 \mathrm{ev} / \mathrm{molecule}$ Figure 3 shows the same 
barrier in configuration space. In order to reach the "valley" corresponding to a complex, the system point must have enough energy to reach the saddle point or "activated state." Although quantummechanical "tunneling" of the system point through the barrier is possible (Refs. 16, 20), the chance of complex formation is still negligible for collision energles much less than $0.30 \mathrm{ev}$.

The existence of a $0.30-\mathrm{ev}$ activation barrier prohibits massive conversion in liquid hydrogen even though radiation might provide a copious source of $\mathrm{H}$ atoms. The fraction of collisions at equilibrium which have more than the minimum energy (E) required for a particular reaction is of the order $\exp (-\mathrm{E} / \mathrm{kT})$, where $\mathrm{k}$ is Boltzmann's constant and $T$ is the absolute temperature. If there were no activation barrier for the formation of the $\mathrm{H}_{3}$ complex, the fraction of collisions which could cause para- to orthomydrogen conversion at liquidhydrogen temperatures would be roughly $\exp (-.015 / .0017)$ or $\exp (-9)$. However, the fraction of collisions with enough energy to overcome the activation barrier is $\exp (-.30 / .0017)$ or $\exp (-176)$. Considering that in liquid hydrogen a molecule undergoes not more than $10^{13}$ collisions per second (of all types), it is clear that in the systems of interest, where the allowable temperature is never much more than $25^{\circ} \mathrm{K}$, no slgnificant conversion cooling can result from equilibrium processes in any reasonable length of time.

The fraction of radiation energy that can be stored in parato ortho-hydrogen conversion through more direct effects is also 
small. Molecules near the path of a high-energy neutron or gamma ray suffer drastic changes, such as lonization and dissociation. While It can be assumed that the return of radiation products to the $\mathrm{H}_{2}$ "ground-state" leads to orthohydrogen in three-fourths of the cases. the effective storage of energy is only $3 / 4 \times 0.015 \mathrm{ev}$ per molecule which is negligible compared to the $15 \mathrm{ev}$ or so that initially went into the destruction of the molecule. And although low-energy neutrons are known to cause orthow to para-hydrogen conversion by virtue of their magnetic moments (Ref。13), the reverse process cannot lead to much energy storage, since in this case there is little energy to store.

Any measurable energy storage by non-equilibrium reactions must involve the energetic secondary products of radiation. Although the energetic $\mathrm{H}$ atoms resulting from disintegration processes (Fig。1) have a good chance of forming a number of $\mathrm{H}_{3}$ complexes before slowing down to equilibrium, the energy absorbed in conversion from groundo state parahydrogen to ground-state orthohydrogen is much less than the energy imparted to the $\mathrm{H}_{2}$ molecules in the decomposition of such complexes. In the early collisions, which absorb most of the H-atom energy, the average energy imparted to $\mathrm{H}_{2}$ molecules is of the order of 1 ev compared to $0.015 \mathrm{ev}$ absorbed in conversion. (Owing to the length of the $\mathrm{H}_{3}$ lifetime, it can be assumed that the line of disintegration is randomly oriented in the center-of-mass system。) The kinetic energy imparted to such molecules must be either distributed as heat or else reabsorbed in the formation of other kinds of 
excited states, since collisions between $\mathrm{H}_{2}$ molecules are ineffective at causing proton spin-filps, $i_{0} e_{0}$, conversions.

Thus, an activation barrier and a relatively small energy-level separation make conversion from ground-state parahydrogen to groundstate orthohydrogen Ineffective, so that one must look to the more robust forms of molecular excitation for a more than minuscule storage of radiation energy in liquid hydrogen. 


\section{DISSIPATION OF STORED ENERGY}

Inspection of Figure $I$ shows that the final appearance of energy distributed among all the local degrees of freedom - that is, of local temperature rise or bolling - depends on the rate of

neutralization of $\mathrm{H}_{3}^{+}$ions, the rate of recombination of $\mathrm{H}$ atoms, and the rate of de-excitation of $\mathrm{H}_{2}$ molecules. If the irradiated portion of the liquid is not rapidly expelled from the system, the energy released in the return of radiation products to the $\mathrm{H}_{2}$ ground state may be transmitted through the volume of liquid by such processes as bubble formation and thermal difiusion. One recent theoretical treatment describes the heat flow along a temperature gradient in a liquid in terms of propagations similar to high-frequency sound waves (Ref。21)。

On the other hand, it is possible that the equipartition of energy does not occur locally; for example, Van Kranendonk (Ref。22) has shown how rotational excitations are transferred from one molevule to another in solid hydrogen and that energy might be transmitted through the liquid in the same way. Whatever the means of long-range energy dissemination and whatever the state of energy partition at which "heating" can be said to have occurred, the expulsion of the immediate products of radiation may substantially reduce the amount of heat that ultimately appears in the system. The present study is restricted to the question of whether the energy going into 
product formation remains locally stored in radiation products for the 20 seconds or so required for the expulsion of the irradiated portion of the Ilquid.

Liquid hydrogen at its normal bolling point is probably the most "gas-like" of the polyatomic liquids. It has recently been shown, for example, that for the analysis of coherent neutron scattering the liquid may be assumed to consist of uncorrelated molecules as in an 1deal gas (Ref。23). Moreover, Raman spectra show that in liquid hydrogen (and even in solld hydrogen), the effect of inter-molecular forces on the $\mathrm{H}_{2}$ vibration frequency is only $0.25 \%$ and that the rotation of the $\mathrm{H}_{2}$ molecule is practically uninhibited (Refs. 24, 22). Hence, there are grounds for supposing that molecules of Ilquid hydrogen carry on more or less independently of each other in their reactions with radiation products, although neither many-body reactions nor energy exchanges between reacting configurations and lattice modes of vibration for the liquid as a whole can definitely be ruled out.

Since the potential energy which must disappear in the neutralization of $\mathrm{H}_{3}^{+}$is readily taken up in the ejection of $\mathrm{H}$ atoms from the unstable complex so formed, the neutralization can occur through a simple 2-body recombination. Therefore, the rate of neutralization depends only on the avallability of electrons and should be relatively high in intense radiation flelds. On the other hand, assuming that liquid hydrogen (or the atom-molecule mixture) is 
gas-like, the recombination of H-atom pairs and the de-excitation of $\mathrm{H}_{2}$ molecules must involve either electromagnetic emission or the transfer of energy to another body. Since both of these processes have a low probability of occurrence compared with 2-ion reactions, the energy-storing capability of liquid hydrogen is determined by reactions involving neutral rather than lonic products of radiation. Since most of the energy inftially associated with $\mathrm{H}$ atoms and excited $\mathrm{H}_{2}$ molecules is lost in only a few collisions with molecules In the supposedly gas-like liquid, it can be assumed that the relatively improbable effects of recombination and de-excitation occur after the radiation products come into kinetic equilibrium with the liquid. Considering that it is an overabundance of energy that makes H-atom recombination difficult, there is no reason to suppose that an energetic $H$ atom is more efficient at causing 3-body recombination than an unenergetic $\mathrm{H}$ atom. In the case of excited $\mathrm{H}_{2}$ molecules, it is true that the de-excitation cross section increases greatly with kinetic energy. However, it is shown in Appendix B (on the basis of Sec。 V) that even for a l-ev molecule the de-excitation cross section is much less than the elastic-scattering cross section and the chance of de-excitation during the 10 collisions or so required for "thermalization" is small. Therefore, the rate at which $\mathrm{H}-a$ tom pairs and $\mathrm{H}_{2}$ molecules return to the $\mathrm{H}_{2}$ ground state is determined by energy transfers and electromagnetic emissions involving products in kinetic equilibrium with the liquid. 
The recombination of H-atom pairs can be regarded as a limiting case of $\mathrm{H}_{2}$ de-excitation, involving an unstable molecule. In general, recombination leads to a bound molecule in an exclted vibrational state, whether the energy released is carried off by a third body or by an electromagnetic emission. Section IV discusses the probable means of 3-body recombination in liquid hydrogen and the significance of theoretically and experimentally determined recombination efficiencies for $\mathrm{H}$ atoms. Recombination by electromagnetic emission is not considered specifically, but the probability of this process is presumably correlated with probabilities of emission by $\mathrm{H}_{2}$ molecules in various excited states.

In a gas-like medium, molecular de-excitation may occur by means of the "superelastic scattering" process in which the excited molecule interacts with one or more molecules or atoms, giving up its excitation energy as relative kinetic energy of recoil. This is the basic mechanism by which stored energy is translated into random molecular motion, or heat. Section $\mathrm{V}$ is devoted to the superelastic de-excitation of $\mathrm{H}_{2}$ molecules in liquid hydrogen. Theoretical data are given in connection with what might be regarded as the opposite extreme from 3-body recombination, namely, the de-excitation of the lowest excited vibrational level of $\mathrm{H}_{2}$.

Radiative emission competes with superolastic scattering in the de-excitation of $\mathrm{H}_{2}$ molecules. Through the emission process, energy is transmitted away from the irradiated portion of the Iiquid. 
The emitted radiations do not directly lead to heat production unless they are absorbed in the walls of the system, since absorption by a molecule of the liquid effectively reproduces the original $\mathrm{H}_{2}$ states at the point of absorption, with negligible momentum transfer. Although emission and reabsorption together might properly come under the heading of energy transport, the transmission of infrared rays to the wall could concelvably be high enough to make the other rate processes irrelevant. Therefore, in Section VI, the probability for both emission and absorption are considered, mainly on the basis of spectral data. 
Blank page 


\section{RECOMBINATION OF H-ATOMS}

\subsection{Three-Body Recombination in Liquid Hydrogen}

Both $\mathrm{H}$ atoms and $\mathrm{H}_{2}$ molecules are avallable to act as third bodies in the recombination of H-atom pairs. Molecules are, of course, much more numerous and are likely to predominate as third bodies in recombination at high temperatures (provided the H-atom concentration is not also extremely high). However, "molecule" recombination probably becomes inoperative at liquid-hydrogen temperatures, whereas H-atom recombination, which involves no activation energy, depends on temperature only insofar as velocities affect the 3-atom collision rate.

An efficacious means of "molecule" recombination would involve the formation of an $\mathrm{H}_{3}$ complex followed by the approach of a second $\mathrm{H}$ atom during the appreciable $\mathrm{H}_{3}$ lifetime (see Table II). However, this cannot occur at Iiquid-hydrogen temperature, since the first $\mathrm{H}$ atom does not have enough energy to surmount the $0.30-e v$ activation barrier. Therefore, at low temperatures molecule recombination can only result from a simultaneous approach of two $\mathrm{H}$ atoms, so that the two atoms together somehow nullify the activation barrier that either would confront singly.

Recombination by the concurrent approach of $\mathrm{H}$ atoms to an $\mathrm{H}_{2}$ molecule may be expected to involve an activation energy (Ref.25). The high activation energy for bimolecular H-atom exchange between 
pairs of $\mathrm{H}_{2}$ molecules (Refo 26) and the repulsive nature of both linear-symmetric and quadratic (square) configurations, as, revealed by calculations of the $\mathrm{H}_{4}$ energy surface (Ref。27), Imply that

$$
\mathrm{H}-\mathrm{H}-\mathrm{H}-\mathrm{H} \text { or } \begin{array}{r}
\mathrm{H}-\mathrm{H} \\
\mathrm{H}
\end{array}
$$

complexes cannot be formed by ground-state molecules and low-energy H-atom pairs. Therefore, it is tentatively concluded that recombination in liquid hydrogen can only occur by means of collisions between three $\mathrm{H}$ atoms.

The classical expression for the number of triple "collisions" per $\mathrm{cm}^{3}$ which involve only $\mathrm{H}$ atoms is (Ref。28)

$$
Z=32 \mathrm{~N}^{3}[\mathrm{H}]^{3} \pi^{3 / 2} \sigma_{\mathrm{H}}^{4} \delta_{\mathrm{H}} \sqrt{\mathrm{RT} / \mathrm{M}}
$$

or

$$
Z=32 \pi^{3 / 2}(H)^{3} \sigma_{H}^{4} \delta_{H} \sqrt{k T} \text {, }
$$

where $\mathrm{N}$ is Avogadro's number, $[\mathrm{H}]$ the hydrogen-atom concentration in moles per $\mathrm{cm}^{3}$, $\mathrm{R}$ the universal gas constant, $M$ the mass of the hydrogen atom in grams per mole, $T$ the absolute temperature, $(H)$ the number of hydrogen atoms per $\mathrm{cm}^{3}, \mathrm{k}$ the Boltzmann constant, $\sigma_{\mathrm{H}}$ the atomic "diameter", and $\delta_{\mathrm{H}}$ the distance to which the third atom must approach the center of mass of the other two atoms for a particular effect to occur. Equation 4-1 simply gives the rate at which H-atom trios assume a certain proximity to each other. Nevertheless, 
a 3-body recombination coefficient, $R_{3}$, can be defined so that

$$
Z=R_{3}(H)^{3} \text {, }
$$

where

$$
R_{3}=32 \pi^{3 / 2} \sigma_{H}^{4} \delta_{H} \sqrt{k T} \text {. }
$$

Unfortunately, the values to use for $\delta_{H}$ are not obvious and may even depend on temperature and mass.

A general expression for $R_{3}$ has been derived (Ref。25) using a semiclassical statistical approach known as the "transition-state" method (Ref。16)。 In this method, it is considered that a collection of particles in equilibrium corresponds to a "gas" of system points moving upon the 3-atom potential-energy surface in configuration space (e.go, Fig。 3 ). Assuming that equipartition of system-point energy prevalis for all degrees of freedom on the surface, the density of system points moving in a given direction at a given point on the surface can be calculated by statistical methods, provided the detailed shape of the surface is known. In this way, the rate of a reaction can be determined without any explicit information on molecular diameters or distances of approach.

Attempts to predict $R_{3}$ by the transition-state method have given a much higher recombination coefficlent than those based on approximate quantum=mechanical treatments of the cross section (Ref。14)。The transitionostate results are considered below, as they are the more conservative from the standpoint of Hoatom expulo sion. (Experimental results are considered in Section 4.2.) 
In general, reactions treated by the transition-state method involve an activation energy, or saddle point, but in $3-\mathrm{H}$ recombination the activation energy is zero. The formal expression obtained by the transition state method is

$$
R_{3}=\frac{2 \pi^{2} I * k T\left(\sigma * h^{2}\right)^{-1} \pi^{2}\left\{1-\exp \left[-h v_{1}(k T)^{-I}\right]\right\}-1(2 \pi m * k T)^{3 / 2} h^{-3} H v}{(2 \pi m k T)^{9 / 2} h^{-9}}
$$

where I*, $\sigma^{*}$, and $\mathrm{m}^{*}$ are, respectively, the moment of inertia, symmetry number, and reduced mass of the $\mathrm{H}-\mathrm{H}-\mathrm{H}$ complex; $\mathrm{m}$ is the mass of a single atom;

$$
\bar{v} \equiv\left(k T / 2 \pi m^{*}\right)^{1 / 2}
$$

is the velocity of the system point at the moment the complex is formed; and $\mathrm{H}$ is a probability integral which for the $\mathrm{H}_{3}$ potential energy surface takes the value

$$
\mathrm{H}=1.64 \times 10^{-8} \mathrm{~h}^{-2}(2 \mathrm{~m} * \mathrm{kT})
$$

The $v_{1}$ are transverse vibration frequencies for the system point at the moment of complex formation and are determined by the degree of curvature transverse to the path of the system particle. All of the quantities in Eq. 4-5 can be reasonably estimated except the $v_{1}$ and $H_{3}$ which are determined by the curvature of the surface in a region where methods for calculating the surface are of dubious validity。 
Equations $4-5,4-6$, and $4-7$ show that in regard to temperature dependence

$$
R_{3} \propto T^{-1 / 2} \prod_{i=1}^{2}\left\{1-\exp \left[-h v_{1} / k T\right]\right\}-1 .
$$

In the case where $h v_{1}<k \mathrm{kT}$, it is seen that $R_{3} \propto \mathrm{T}^{3 / 2}$. The disagreement in temperature dependence between Eq.4-5 and the "proximity" Eq. 4-1 can be ascribed to a velocity dependence for $\delta_{H}$ with regard to recombination collisions.

The coefficient $R_{3}$ has been calculated for room temperature by Eyring, Gershinowitz, and Sun (Ref。25). The value taken for both $v_{1}$ and $v_{2}$ was $100 \mathrm{~cm}^{-1}$ or $3 \times 10^{12} \mathrm{sec}^{-1}$, corresponding to $h v_{1} / \mathrm{kT}^{\mathrm{T}}=$ 0.5 for room temperature. This choice of $v_{1}$ was the result of a compromise between higher values of $v_{1}$ Indicated by the calculated $\mathrm{H}_{3}$ surface and lower values of $v_{1}$ needed to give what was thought to be the right dependence on mass. An experimental comparison between $R_{3}$ for ordinary hydrogen and for deuterium (Ref。28) had Indicated an $\mathrm{m}^{-1 / 2}$ dependence, which is obtained from Eq. 4.5 when $h v_{1} \ll R T$, and

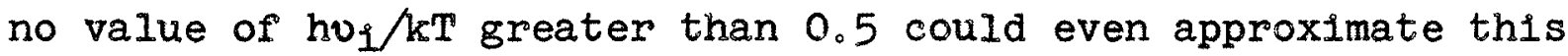
condition. The use of this value could only lead to an order of magnitude error if the actual $v_{1}$ were much below $100 \mathrm{~cm}^{-1}$; yet enough confidence was placed in the calculated $\mathrm{H}_{3}$ surface that this was thought unlikely。

The value obtained for $R_{3}$ based upon the rather ad hoc choice of $v_{1}$ was $3.1 \times 10^{15} \mathrm{~cm}^{6} \mathrm{~mole}^{-2} \mathrm{sec}^{-1}$ or $8.5 \times 10^{-33} \mathrm{~cm}^{6}$ atom $^{-2} \mathrm{sec}^{-1}$ 。 
In contrast, the value calculated by an approximate quantummechanical approach (Ref。 14 ) is $R_{3}=10^{-42} \mathrm{~cm}^{6}$ atom ${ }^{-2} \mathrm{sec}^{-1}$. Adjustment of the higher value to $20^{\circ} \mathrm{K}$ by the use of $\mathrm{Eq}$. 4-8 reduces it by a factor of 1.67 , giving $R_{3}=5 \times 10^{-33} \mathrm{~cm}^{6}$ atom ${ }^{-2} \mathrm{sec}^{-1}$. Assuming this coefficient is correct, it can be used to estimate the fraction of H atoms expelled from the propulsion system of interest. The fraction of $\mathrm{H}$ atoms which recombine per second at a point where the concentration is $(H)$ can be written as

$$
F=R_{3}(H)^{3} /(H)=R_{3}(H)^{2} \text {. }
$$

It is seen that $F$ is a small fraction as long as (H) does not exceed $1015 \mathrm{H}$ atoms per $\mathrm{cm}^{3}$.

A crude estimate of the radiation level corresponding to $10^{15}$ $\mathrm{H}$ atoms per $\mathrm{cm}^{3}$ at the instant of emission for the expulsion rate of interest can be made without writing a differential equation to describe the production and recombination as a function of position (such an equation is found to be both nonlinear and inhomogeneous). Taking an energy-flux relaxation length of $100 \mathrm{~cm}$ and an effective exposure time of 20 seconds for volume elements of liquid, the total energy deposited per $\mathrm{cm}^{3}$ of element is roughly of the order of $(100)^{-1}\left(\phi_{0} e^{-1}\right)(20)$, where $\phi_{0}$ is the incident energy flux. According to Figure 1, 5/33 atoms are produced for each electron volt deposited. Therefore, for low rates of recombination the average concentration produced by the flux $\phi_{0}$ before the liquid element is expelled is 
$(100)^{-1}\left(\phi_{0} e^{-1}\right)(20)(5 / 33)$, or $0.01 \phi_{0}$ (with $\phi_{0}$ in ev). Setting this equal to 1015 atoms per $\mathrm{cm}^{3}$, it is seen that the fraction of recombining $\mathrm{H}$ atoms is small as long as the incident energy flux is not much greater than $10^{17} \mathrm{ev} / \mathrm{cm}^{2}-\mathrm{sec}$, provided of course that the calculated value of $R_{3}$ is right. This would mean, for example, that most $\mathrm{H}$ atoms would be expelled unrecombined for fast-neutron fluxes up to $10^{11}$ neutrons $/ \mathrm{cm}^{2}-\mathrm{sec}$. Although this is a higher flux than would be found near the core of many conventional reactors, it must be considered as moderate from the standpoint of fluxes expected for nuclear space vehicles. However, since $\mathrm{Eq}$. $4-5$ gives the number of $\mathrm{H}_{2}$ recombinations of all types, including those which lead to highly excited $\mathrm{H}_{2}$ states, the value used for $R_{3}$ might be consistent with the expulsion of a large fraction of unpartitioned energy for much higher flux levels。

The calculation of $R_{3}$ by Eq. $4-5$ involves the $H_{3}$ potential energy surface not only through the choice of the $v_{1}$ but through the probability integral $H_{0}$ Considering that the value of $v_{1}$ given by the surface is not consistent with the experimental mass dependence (which may be correct even though part of the experiment was probably misinterpreted, as will be seen in Eq. 4-2), it is quite conceivable that the value of $\mathrm{H}$ given in Eq. 4-7 is wrong by orders of magnitude (as is indicated by the quantum-mechanical approximation). However, direct experimental determinations of $R_{3}$, discussed in Eq. 4-2, show that the calculated value is at least not too small. 
4.2 Experimental Results on Recombination

Attempts in 1934 to measure the hydrogen atom recombination coefficient experimentally resulted in an almost classic conundrum. Amdur (Ref。28) found that at room temperature $H$ atoms are ten times more effective than $\mathrm{H}_{2}$ molecules as third bodies in the recombination of H-atom pairs. Steiner (Ref。29) came to just the contrary conclusion, namely, that $\mathrm{H}_{2}$ molecules are at least ten times more effective than $\mathrm{H}$ atoms. Both experiments were remarkable for the internal consistency of their data. (An experimental value for $R_{3}$ cited in Reference 14 is approximately that obtained by Amdur and is presumably based on his experiment or one similar to it.)

Both Amdur and Steiner used a discharge tube to obtain $H$ atoms from hydrogen gas, with Amdur reporting degrees of hydrogen dissoclation ranging from $18 \%$ to $69 \%$. In each case, the atom-molecule mixture was allowed to drift down a recombination tube. The methods used to determine the Hoatom concentration at various positions along the tube were radically different in the two experiments. Amdur measured the amount of heat absorbed by a "catalyst-calorimeter" as a function of position. The heat absorbed was assumed to be proporm tional to the number of catalyzed Hoatom recombinations and, there fore, to the number of $H$ atoms which had escaped natural recombination in drifting to the point of measurement. On the other hand, Steiner determined the relative Hoatom concentration by spectroscopy, presumably by the observation of Lyman-line absorption (which is different for $\mathrm{H}$ and $\mathrm{H}_{2}$ )。 
Amdur found that the rate of change of heat absorption in the calorimeter (as a function of position) was proportional to the cube of the heat absorbed. Assuming that all the heat generated was due to the recombination of H-atom pairs, he inferred that the third bodies involved in the recombination were predominantly $\mathrm{H}$ atoms. However, Steiner's spectroscopic observations showed that the rate of change of Haatom concentration was proportional to the square of the H-atom concentration, which implied that the third bodies were predominantly not $\mathrm{H}$ atoms and were therefore $\mathrm{H}_{2}$ molecules.

An explanation for the disagreement which seems plausible in light of the high degree of dissociation is that in both experiments the $\mathrm{H}_{2}$ molecules present were in highly excited vibrational states. A discharge capable of dissociating a large fraction of the molecules would have initially left most of the remaining molecules with excitation energies comparable to the dissociation energy; moreover, the Iffetimes of excited H2 states against emission are known to be extremely long (see Sec.VI). It is thereby proposed that equipartition of energy did not occur in either experiment. This supposition o that the atom-molecule mixture was at "room tempera. ture ${ }^{8}$ only with regard to translational energy - becomes credible when it is considered that provisions were made to prevent the recombination of $\mathrm{H}$ atoms at the walls of the system and that this would also have inhibited the de-excitation of $\mathrm{H}_{2}$ molecules at the walls。 
The above hypothesis can be reconciled with all the experimental data. Since few molecules could have been raised to excited electronic states, Steiner's spectroscopic examination would not have revealed any $\mathrm{H}_{2}$ excitation. Steiner's observation that the third bodies in the experiment were $\mathrm{H}_{2}$ molecules is quite plausible, considering that a long -1 ived $\mathrm{H}_{3}$ complex could easily be formed from an $\mathrm{H}$ atom and a highly excited $\mathrm{H}_{2}$ molecule (since, due to internal excitation, the "kinetic energy" of the system point would be much higher than the activation energy). The energetic $\mathrm{H}_{3}$ complex could, in turn, easily react with a second $H$ atom. The two processes in sequence should be much more likely than the simultaneous collision of three $\mathrm{H}$ atoms. If this 1 s the case, the recombination rate should depend on the square of the atomic concentration as observed by Steiner.

Even though $\mathrm{H}_{3-\mathrm{complex}}$ formation may be a primary step in recombination, relatively $\mathrm{few}$ of the $\mathrm{H}_{3}$ complexes that are formed have time to react with another $H$ atom before disintegrating. (The actual $\mathrm{H}_{3}$ lifetimes are much shorter than those shown in Table II, because of the probable formation of a highly excited $\mathrm{H}_{2}$ state.) Therefore, the principal means by which excitation energy is converted into kinetic energy should be the de-excitation of $\mathrm{H}_{2}$ states through the formation and decomposition of $\mathrm{H}_{3}$ complexes. Since $\mathrm{H}_{3}$ decomposition leading to a completely de-excited molecule is unlikely and since an eventual balance should be reached between 
kinetic energy production through $\mathrm{H}_{3}$ decomposition and the formation of new excited $\mathrm{H}_{2}$ molecules by recombination, the depopulation of excited $\mathrm{H}_{2}$ states should have only a small effect on the recombination rates as observed by Steiner. On the other hand, the de-excitation of highly excited $\mathrm{H}_{2}$ molecules by the formation and decomposition of $\mathrm{H}_{3}$ would be almost all that could be observed in the calorimetric method used by Amdur, considering that $\mathrm{H}$ atoms can certainly bring about the de-excitation of $\mathrm{H}_{2}$ molecules more easily than the recombination of Heatom pairs.

Using the calorimetric method, it would be impossible to distinguish between $\mathrm{H}$-atom pairs and highly excited $\mathrm{H}_{2}$ molecules. Hence, It is logical that the depopulation of excited $\mathrm{H}_{2}$ states by the formation and decomposition of $\mathrm{H}_{3}$ complexes should have been interpreted by Amdur is recombination with $\mathrm{H}$ atoms as third bodies. Amdur actually did observe the effect of reactions involving trios of $\mathrm{H}$ atoms, in the sense that a highly excited $\mathrm{H}_{2}$ molecule is composed of two $\mathrm{H}$ atoms which are barely bound together. However, the rate of return to low-lying $\mathrm{H}_{2}$ states was much different than what would have been obtained from the recombination of free $H$ atoms, owing to the much smaller "entropy decrease" required for the de-excitation process。

It is concluded that Amdur's findings are based upon an incorrect interpretation of the data and that his value of $R_{3}$ and the one cited In Reference $14\left(\sim 3 \times 10^{-32} \mathrm{~cm}^{6}\right.$ atom $^{-2} \mathrm{sec}^{-1}$ in each case) are too high. 
Steiner's conclusions are assumed to be right, except that the recombination coefficient he obtained for molecules as third bodies should actually apply only to excited $\mathrm{H}_{2}$ molecules. One would hope that the spectroscoplc observations were precise enough to give the effectiveness of the relatively improbable "3-atom" recombination process, since the quantity of interest in the case of liquid hydrogen is $R_{3}$, the recombination coefficient for $H$ atoms as third bodies.

Unfortunately, Steiner's data only provided an upper limit on $R_{3}$ for room temperature. Steiner interpreted his results in terms of each of the following separate assumptions regarding $R_{3}$ : (1) $R_{3}$ is equal to $R_{2}$, the recombination coefficient for molecules as third bodies; (2) $R_{3}$ is one-tenth as large as $R_{2}$; (3) $R_{3}$ is completely negligible compared to $R_{2}$. The first assumption, of course, proved to be wholly inconsistent with the data. The second and third assumptions were both consistent with the data, the arbitrariness arising from the unknown rate of wall recombinations. For $R_{2}$, Steiner got $1.3 \times 10^{16} \mathrm{~cm}^{6} \mathrm{~mole}^{-2} \mathrm{sec}^{-1}$. This means that $R_{3}$ for room temperature is somewhere between 0 and $1.3 \times 10^{15} \mathrm{~cm}^{6} \mathrm{~mole}^{-2} \mathrm{sec}^{-1}$ 。

It is seen that the room temperature, $R_{3}$, calculated by Eyring et al. $\left(3.1 \times 10^{15} \mathrm{~cm}^{6} \mathrm{~mole}-\mathrm{e}^{-2} \mathrm{sec}^{-1}\right)$ is too large and, on the basis of either theory or experiment, quite possibly far too large. If the method used in Eq. $4-1$ to adjust $R_{3}$ to liquid-hydrogen temperature is valid, then $R_{3}$ for liquid hydrogen is not more than (1.3/3.1) $\left(5 \times 10^{-33}\right) \mathrm{cm}^{6}$ atom $^{-2} \mathrm{sec}^{-1}$ and is possibly much less. This means that 
the maximum $\mathrm{H}$-atom concentration permitting a high rate of $\mathrm{H}$-atom expulsion in the systems of interest is concelvably much greater than $10^{15}$ atoms $/ \mathrm{cm}^{3}$ : for example, $10^{20}$ atoms $/ \mathrm{cm}^{3}$ if the theoretical $R_{3}$ of Reference 14, 10-42 $\mathrm{cm}^{6}$ atom $^{-2} \mathrm{sec}^{-1}$, is correct. The available experimental data are, in fact, consistent with predominant H-atom expulsion at arbitrarily high radiation levels.

The assumption that the values of $R_{3}$ for liquid hydrogen and for hydrogen gas at room temperature are related by Eq. 4-8, is of course subject to question. The effective $R_{3}$ for liquid hydrogen would be larger than expected if $\mathrm{H}$ atoms in the liquid were constrained to move in certain planes or channels by lattice effects. On the other hand, $R_{3}$ at $20^{\circ} \mathrm{K}$ could be lower than is indicated by the use of Eq. 4-8, because of wave-mechanical interference; a low-energy system point dropping into a "basin" in configuration space is subject to reflection after the fashion of a light wave entering air from a denser medium (Ref. 16). Effects of this sort will not be considered here。 


\section{Blank page}


V. SUPERELASTIC DE-EXCITATION OF H2 MOLECULES

\subsection{Superelastic Process in Liquid Hydrogen}

The amount of energy stored in excited $\mathrm{H}_{2}$ states through lonic and dissociative processes is relatively small (see Fig。 I), However, excited $\mathrm{H}_{2}$ molecules are also formed by inelastic colitisions with the energetic $\mathrm{H}$ atoms or $\mathrm{H}_{2}$ molecules produced by radiation-induced reactions and by the recombination of Haatom pairs. (Electromagnetic excitation is considered in Section VI.) Therefore, the process of de-excitation could have more influence on the energy-storing capabilities of liquid hydrogen than the recombination process.

Aside from radiative emission, de-excitation results from superelastic scattering between the excited molecule and another molecule or atom. On an individual basis, H atoms should be more effective than ground-state $\mathrm{H}_{2}$ molecules at bringing about de-excitation. Thus, in the experiments described in Section IV, where the dissociation was of the order of $50 \%$, it was assumed that $\mathrm{H}_{2}$ de-excitation was mainly due to $\mathrm{H}$ atoms. But in the propeliant systems of interest, there will be around $10^{4}$ times as many molecules avaliable as atoms (assuming the maximum rate of H-atom recombination); and, owing to the fact that a molecule-molecule system point surmounts an activation barrier almost as readily as an atommolecule system point in reactions involving an excited molecule, the efficiency for molecule-molecule demexcitation should not be less than that for atom-molecule de-excitation by a factor as large 
as $10^{4}$. Hence, it is provisionally assumed that the predominant means of superelastic de-excitation is through molecule-molecule reactions.

It is a commonly accepted fact that energy exchanges between Internal and external degrees of freedom occur only with difficulty (Ref. 30, p. 293: Ref. 31, p. 143) In the sense that only a minute fraction of collisions are favorable for de-excitation at ordinary temperatures. At liquid-hydrogen temperatures, where the distance of closest approach between molecules is relatively large and where the internal molecular fields are only slightly perturbed, de-excitation should be much more difficult still. In other words, the "relaxation time", or mean lifetime against de-excitation, should be much longer in liquid hydrogen than in hydrogen gas at the same density. Whether it is longer than the 20-second expulsion time depends on the superelastic scattering cross section averaged over a Boltzmann distribution.

Ordinarily, experimental information on relaxation times can be obtained through measurements of the dispersion and absorption of sound, using interference techniques. Thus, the rotational relaxation time for $\mathrm{H}_{2}$ and the vibrational relaxation time for $\mathrm{CO}_{2}$ have been obtained under standard conditions (Ref". 32)。 However, the first vibrational level of $\mathrm{H}_{2}$ is too high to be readily accessible by ultrasonics. Moreover, as there is no evident way of determining the temperature dependence of the relaxation process, any acoustical measurements of the vibrational rate in the liquid would have to be 
carried out at low temperatures. Since no such experiments appear to have been made, one is forced for the present to rely on a relaxation time based on calculated superelastic scattering cross sections as a function of energy. (Direct cross-section measurements are not out of the question, but the target problem would be formidable。)

\subsection{Wave-Mechanical Treatment of Inelastic and Superelastic} Scattering Between $\mathrm{H}_{2}$ Molecules

Both the internal force that holds the $\mathrm{H}_{2}$ molecule together and the external force between pairs of $\mathrm{H}_{2}$ molecules that are not colliding too violently are relatively well known. Hence, there are grounds for supposing that, for low-collision energies, the energy-dependent superelastic cross section can be predicted reasonably well by theoretical methods. A direct wave-mechanical treatment is more sultable for this purpose than the illustrative transition-state method, which, in any case, requires a wave-mechanical determination of energy surfaces. A method has been developed by Bauer and Salkoff (Ref。33) which, in principle, can be used to give either the inelastic or superelastic cross sections for collisions between two $\mathrm{H}_{2}$ molecules. The procedure is important enough to outline here. The baslc features of the general formalism of Salkoff and Bauer can be enumerated as follows:

1. The nuclei of the colliding molecules are assumed to move so much more slowly than the electrons that electronic equilibrium prevalis at each instant during the collision (this is known as the Born-Oppenheimer approximation).

2. The nuclear motions are described by wave mechanics. 
3. One of the molecules - the one that is not to change 1ts vibrational state - is treated as a polnt mass; the other is regarded as a pair of $\mathrm{H}$ atoms.

4. The force between atom and "point" molecule is assumed to have the same spatial dependence for each atom; i.e., the atom nearest the molecule does not interfere with the interaction between the molecule and the more remote $\mathrm{H}$ atom.

5. The atom-molecule and atom-atom forces are treated on different bases; a Morse potential is used to describe the "external" atom-molecule interaction, whereas. the attractive "Internal" atom-atom force is based upon known properties of the $\mathrm{H}_{2}$ molecule.

6. The "change" in the net external potential (the sum of the two atom-molecule potentials) arising from the distortion of the average separation of the $H$ atoms, which occurs because of the proximity of the molecule, is assumed to be relatively small; 1 。e, it is taken as a perturbation.

7. Only purely vibrational transitions are considered (although the formalism could easily be extended to include rotational excitation). Therefore, all noncentral features of the total potential energy of the system are ignored; the center of mass of the "point" molecule is assumed to lie on a line with the two atoms during the brief collisions interval (although the direction of relative motion is not assumed to be along this line in general; $1 . e_{0}$, the angular momentum is not necessarily zero).

Consider the system as made up of atom $A$, atom $B$, and molecule $C$ from left to right in accordance with Item 7 . Let $\underline{R}$ be the position vector of the center of mass of the entire system with respect to an arbitrarily placed coordinate system. Then,

$$
\underline{R}=\left(M_{A} \underline{r}_{A}+M_{B} \underline{r}_{B}+M_{C} \underline{r}_{C}\right) /\left(M_{A}+M_{B}+M_{C}\right)_{9}
$$

where the $M_{A}=M_{B}$ is the atomic mass and $M_{C}=2 M_{A}$ is the molecular 
mass. The vector between the "point" molecule and the center of mass of the atoms is

$$
\underline{r}=\underline{r}_{C}-\left(M_{A} \underline{r}_{A}+M_{B} \underline{r}_{B}\right) /\left(M_{A}+M_{B}\right)
$$

and the vector between the atoms can be written as

$$
e=\underline{r}_{A}-\underline{r}_{B} \text { 。 }
$$

The reduced masses corresponding to $\underline{\rho}$ and $\underline{\underline{r}}$ are, respectively,

$$
M_{1}=M_{A} M_{B} /\left(M_{A}+M_{B}\right)
$$

and

$$
M_{e}=M_{C}\left(M_{A}+M_{B}\right) /\left(M_{A}+M_{B}+M_{C}\right) \text {, }
$$

where the subscripts $i$ and e refer to the "Internal" coordinate $\underline{p}$ and "external" coordinate $\underline{r}_{\text {. }}$

As observed from a frame at rest with respect to the center of mass, $\underline{R}$, the total energy of the system is then

$$
H(\underline{r}, \underline{\rho})=\frac{1}{2} M_{1} \dot{\rho}^{2}+\frac{1}{2} M_{e} \dot{r}^{2}+V(\underline{r}, \underline{\rho})
$$

where $V\left(\underline{r}_{2} \underline{l}\right)$ is the total potential energy. In terms of wave mechanics,

$$
H(\underline{r}, p)=\frac{-\hbar^{2}}{2 M_{1}} \Delta_{\rho}^{2}-\frac{\hbar^{2}}{2 M_{e}} \Delta_{r}^{2}+V(\underline{r}, \underline{\rho})
$$

and the Schrodinger equation is

$$
H(\underline{r}, p) \Psi=E \Psi
$$


where $E$ is the total energy eigenvalue. In view of Item $7, V(\underline{r}, \underline{\rho})$ depends only on the magnitude of $\underline{r}$ and $\rho$. Then from Eqs. 5-6 and 5-7,

$$
-\frac{\hbar^{2}}{2 M_{1}} \Delta_{\rho}^{2} \Psi-\frac{\hbar^{2}}{2 M_{e}} \Delta_{r}^{2} \Psi+V(r, \rho) \Psi=E \Psi \text {. }
$$

In accordance with Item 6 , the total potential may be written

$$
v(r, p)=v_{1}^{o}\left(r_{0}, \rho\right)+v_{e}^{o}\left(r_{,} p_{0}\right)+v^{\prime}(r, p)
$$

where the subscript and superscript $\circ$ refers to the values that would obtain for an external separation $\underline{r}_{0}$, assuming no distortion of the field between $H$ atoms $V_{1}\left(r_{0}, p\right)$. The "unperturbed" external potential, $V_{e}^{0}\left(r, p_{0}\right)$, is the sum of the potentials between the two $H$ atoms and the "point" molecule for the average internal separation Pos

$$
V_{e}^{O}\left(r, p_{0}\right)=M\left(r+p_{0} / 2\right)+M\left(r-p_{0} / 2\right),
$$

where $M$ represents the functional form of the Morse potential in accordance with Item 5. The perturbation term is given by

$$
V^{\prime}(r, p)=\left[\frac{\partial}{\partial \rho} V_{e}^{O}(r, p)\right]_{p_{0}} d \rho=\left[\frac{\partial}{\partial \rho} V_{e}^{O}(r, p)\right]_{p_{0}}\left(p-p_{0}\right) \cdot(5-11)
$$

The "unperturbed" equation is obtained by substituting Eq. 5-9 into Eq. 5-8, with V' set equal to zero. First order perturbation theory then gives the desired cross section in terms of $V^{\prime}$ and the solutions to the unperturbed equation. (The Born approximation would be obtained by ignoring $V^{\prime}$ entirely and taking $V_{e}^{0}$ as the perturbation; 
however, this cannot be done here, since it is the coupling term $V^{\prime}$ that leads to transitions.) The "unperturbed" solution to Eq. 5-8 can be written

$$
\Psi_{0}=\phi_{j}(p) \psi_{j}(r)
$$

where the $\phi_{j}$ and $\psi_{j}$ are the solutions of

$$
\left(-\frac{\hbar^{2}}{2 M_{1}} \Delta_{p}^{2}+v_{1}^{o}\left(r_{0}, p\right)-E_{j}\right) \phi_{j}(p)=0
$$

and

$$
\left(-\frac{\hbar^{2}}{2 M_{e}} \Delta_{r}^{2}+v_{e}^{o}\left(r, p_{0}\right)-\frac{\hbar^{2} k^{2}}{2 M_{e}}\right) \psi_{j}(\underline{r})=0
$$

The internal Eq.5-13 is simply the wave equation for the hydrogen molecule. The energy eigenvalues are given by

$$
E_{j}=h \omega_{j},
$$

where the $\omega_{j}$ are the permissible frequencies. The solutions $\phi_{j}$, In accordance with Item 5, are based upon experimental data. It is found that the internal potential can be expressed as (Ref. 34 . p. 304)

$$
V_{1}^{o}(p)=U_{0}\left\{\exp \left[-2\left(p-p_{0}\right) / a^{\prime}\right]-2 \exp \left[-\left(\rho-p_{0}\right) / a^{\prime}\right]\right\},
$$

which, for not too high levels of vibration, has the (normalized) solution

$$
\phi_{j}=\left(\frac{1}{4 \pi}\right)^{\frac{1}{2}}\left(\frac{\alpha}{\pi^{\frac{1}{2}} 2^{J_{!}}}\right)^{\frac{1}{2}} \quad \frac{1}{\rho} \quad H_{j}\left[\alpha\left(\rho-\rho_{0}\right)\right] \exp \left[-\frac{1}{2} \alpha^{2}\left(\rho-\rho_{0}\right)^{2}\right],
$$


where, for the case of no rotation,

$$
\alpha=\left[\frac{2 M_{1}}{\hbar^{2}} \frac{U_{0}}{a^{2}}\right]^{1 / 4}
$$

and $\mathrm{H}_{\mathrm{f}}$ is the $\mathrm{fth}$ Hermite polynomial.

Spectroscopic data (Ref。35) give $a^{\prime}=5.40 \times 10^{-9} \mathrm{~cm}$ and $U_{0}=7.97 \times 10^{12}$ ergs.

The external Eq. 5-14 describes the relative motion of the centers of mass of the two molecules with respect to a frame moving with $\underline{R} ; \hbar^{2} k^{2} / 2 M_{e}$ is the relative kinetic energy of the molecules before collision. The external potential is given by Eq.5-10, where

$$
M(x)=v_{e}^{\circ}\{\exp [-2 a(x-d)]-\exp [-a(x-d)]\} 。
$$

Bauer and Salkof $\mathrm{f}$ used $\mathrm{U}_{\mathrm{e}}^{\circ}=1.82 \times 10^{-14} \mathrm{ergs}, a=1.73 \times 10^{8} \mathrm{~cm}^{-1}$, and $\mathrm{d}=3.00 \times 10^{-8} \mathrm{~cm}$.

The unperturbed solutions of Eq, 5-8 are obtained by expanding the solutions of Eq. 5-14 in terms of Legendre polynomials and substituting into $\mathrm{Eq}$. 5-12. In this way, an unperturbed wave function is obtained for a system having a given internal vibration level and a given collision energy. Analysis of the asymptotic magnitudes of the scattered waves shows that the cross section for transition from unperturbed state $\Psi_{0}$ to unperturbed state $\Psi_{f}$ is

$$
\sigma_{f o}(\theta, \varnothing)=\frac{k_{f}}{k_{0}} \frac{M_{e}^{2}}{4 \pi^{2} h^{4}}\left|\int \Psi_{o} v^{\prime} \Psi_{f} d \tau\right|^{2},
$$

where $\theta$ and $\varnothing$ give the direction of scatter and the integration is 
over all the coordinates. Jaing the Legendre expanded form of Eq. 5-12, integrating over all directions and making use of the orthogonality properties gives, for the total cross section,

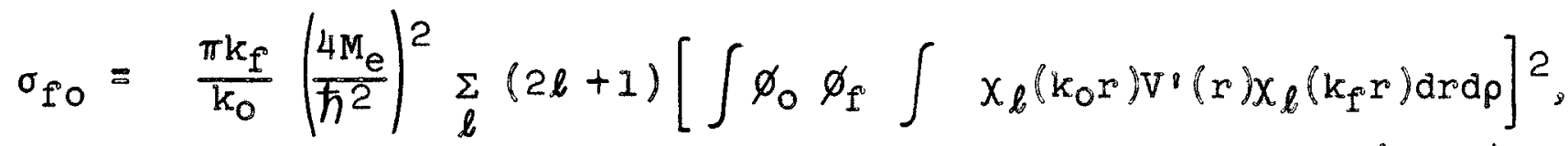
(5-19)

where $V^{0}$ is given by Eq. $5-11$ and $x_{b}$ is the solution of

$$
\frac{d^{2} x_{l}}{d r^{2}}+\left[k_{j}^{2}-\frac{2 M_{e}}{\hbar 2} \quad v_{e}^{0}\left(r, p_{0}\right)-\frac{l(l+1)}{r^{2}}\right] x_{l}=0
$$

The plece de resistance of the problem is the solution of Eq. 5-20。 Bauer and Salkof obtained $x_{\ell}$ numerically, using a highspeed digital computer. A starting point for the integration was obtained by taking the WKB solution (Ref。34) for Eq. 5-20 in the asymptotic region, where the coefficient of $X_{b}$ varies slowly and the WKB method is valid. Tests were performed to ensure that no range of $r$ contributing significantly to the cross section was neglected. A check was also made to determine whether the number of values of $l$ considered was sufficient。

The cross section for the inelastic excitation of the first vibrational level of $\mathrm{H}_{2}$ was calculated as a function of energy. The results are shown in Figure 4 (the subscripts on $\sigma$ being omitted for convenience). Although the possibility of rotational excitation was not considered, it is reasonable to assume that the probability 
of vibrational excitation is of the same order of magnitude whether or not a simultaneous rotational excitation occurs. The cross sections for the reverse process of superelastic de-excitation were not calculated directly. However, they are related to the inelastic cross sections as described in Appendix $B$, and the desired relaxation time can be obtained from the inelastic cross sections as shown in Section 5.3.

5.3 Relaxation Time of the First Vibrational H2 State in Liquid Hydrogen

The relaxation time, or mean lifetime, of an exclted state in a gas-like medium, where only the ground state, 0 , and the first excited state, 1, are accessible to molecules, is given by (Ref。36)

$$
\tau_{10}=1 / \mathrm{f}_{10}
$$

where $f_{10}$ is the probability per unit time of the molecule undergoing the $1 \rightarrow 0$ transition. Such transitions are induced by thermal collisions. Unexcited molecules have a probability per unit time, $f_{01}$, of undergoing the $0 \rightarrow 1$ transition. At equilibrium, the rates of excitation and de-excitation must be equal. Therefore, if $\bar{n}_{0}$ and $\bar{n}_{1}$ are the numbers of unexcited and excited molecules at equilibrium,

$$
f_{01} \bar{n}_{0}=f_{10} \bar{n}_{1}
$$

For a system of $n$ particles and a phase space of just two cells, it can be shown (Ref.37, p. 289) that, if the energies of the cells 


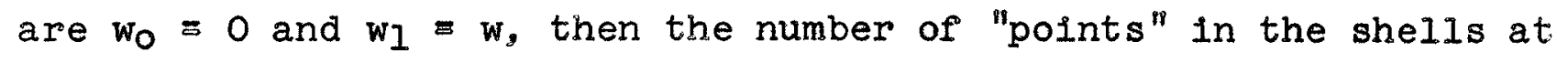
equilibrium are, respectively,

and

$$
\bar{n}_{0}=n\{1+\exp [-w / k T]\}-1
$$

$$
\bar{n}_{I}=n\{1+\exp [\mathrm{w} / \mathrm{kT}]\}-1 \text {. }
$$

In the case of vibrational excitation.

$$
\text { w }: \text { hv。 }
$$

Thus, the ratio of excited to unexcited states at equilibrium is

$$
\bar{n}_{1} / \bar{n}_{0}=\{1+\exp [-h v / k T]\} /\{1+\exp [h v / k T]\}
$$

and, from Eq. 5-22,

$$
\mathrm{f}_{10}=\mathrm{f}_{01}\{1+\exp [\mathrm{hv} / \mathrm{kT}]\} /\{1+\exp [-\mathrm{hv} / \mathrm{kT}]\} \text { 。 }
$$

The number of excitations per $\mathrm{cm}^{3}$ per sec due to collisions where the relative velocity is between $v$ and $v+d v$ is, using $E q$ 。 $5-23$,

$$
\bar{n}_{O} d f_{O I}=\bar{n}_{O} \eta(v) d v v \sigma_{01}(v) n\{I+\exp [-h v / k T]\}-I,(5-28)
$$

where $n$ is now regarded as the total number of molecules per unit volume and $\eta(v) d v$ is the fraction of collisions where the relative velocity is in the interval $d v$. Then, from Eqs. 5-27 and 5-28, $\mathrm{df}_{10}=\eta(\mathrm{v}) \mathrm{dv} \mathrm{v \sigma ol}_{01}(\mathrm{v})\{1+\exp [\mathrm{hv} / \mathrm{kT}]\} /\{1+\exp [-\mathrm{hv} / \mathrm{kT}]\}_{2}$ 
At equilibrium, Eq.5-29 holds rigorously。 For hydrogen, ho is so large compared to $\mathrm{kT}$ for reasonably low temperatures that the denominator in Eq. 5-29 can be set equal to unity. This says that the depletion of unexcited states due to excitation by thermal collisions has almost no effect on the probability of de-excitation. Therefore,

$$
d f_{10}=\eta(v) d v \quad v \sigma_{0 I}(v)\{I+\exp [h v / k T]\}
$$

whether or not the concentration of exclted states is at equilibrium for the temperature $T$. The total probability of de-excitation is

$$
f_{10}=n k_{10}\{1+\exp [h v / k T]\} \text {, }
$$

where $k_{10}$ is obtalned by averaging $v \sigma_{01}(v)$ over a Boltzmann distribution of relative velocities. Then, from Eq. 5-21,

$$
\tau=\left\{n k_{10}[1+\exp (h v / k T)]\right\}-1 \text {. }
$$

Salkoff and Bauer evaluated $\tau$ as a function of temperature, using the $\sigma_{01}(v)$ shown in Figure 4. Their results are show in Figure 5. For some reason, the value of $n$ used was not given. Under such circumstances, it could ordinarily be assumed that the "sec" units indicated for $T$ actually referred to "atomosphereseconds $^{88}$ - In other words, that the results corresponded to the density of hydrogen at one atmosphere of pressure for the temperature considered. However, it appears from a rough check of the results that the value of $n$ used was independent of temperature and of the order $10^{18}$ molecules $/ \mathrm{cm}^{3}$. 


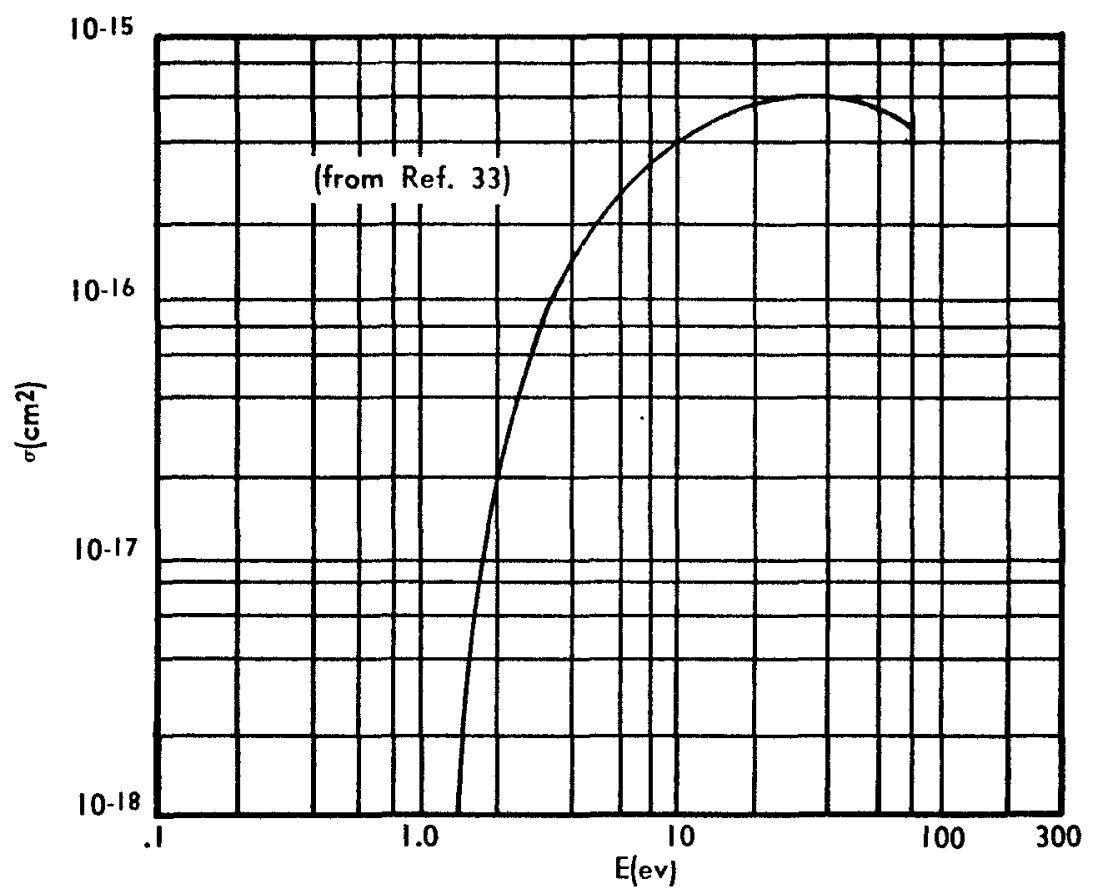

Figure 4. Cross Section for the Excitation of the First Vibrational $\mathrm{H}_{2}$ Level by Inelastic Scattering between a Pair of $\mathrm{H}_{2}$ Molecules

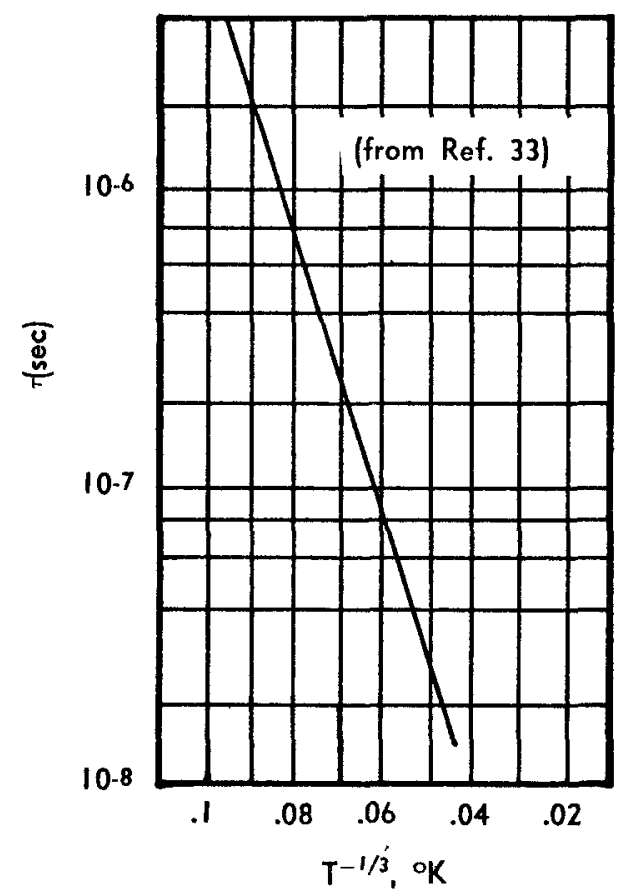

Figure 5. Vibrational Relaxation Time for the First $\mathrm{H}_{2}$ Level at an Unspecified Low Density 
A curve-fit which accurately reproduces both the magnitude and slope of the calculated relaxation time over the calculated range is

$$
\tau=6.41 \times 10^{-11} \exp \left(115.2 \mathrm{~T}^{-1 / 3}\right)
$$

If this temperature-dependence can be extended to liquid-hydrogen temperatures, then it is found that $\tau=1.74 \times 10^{8} \mathrm{sec}$ at $20^{\circ} \mathrm{K}$ and the molecular density used by Bauer and Salkoff. Assuming their value of $\mathrm{n}$ was $10^{18}$ molecules $/ \mathrm{cm}^{3}$ and using the molecular density of liquid hydrogen - i.e., 2.11 $\times 10^{22}$ molecules $/ \mathrm{cm}^{3}$ - it is seen from Eq. 5-32 that for liquid hydrogen

$$
\tau=\left(10^{18} / 2.11 \times 10^{22}\right)\left(1.74 \times 10^{8}\right)=8271 \mathrm{sec} .
$$

Thus, contingent upon the admittedly Brobdingnagian extrapolation of calculated values, the relaxation time of the first excited vibrational level in liquid hydrogen is of the order of 100 minutes. 5.4 Applicability of the Isolated-Binary-Collision Assumption for Liquid Hydrogen

It was tacitly assumed in Section 5.3 that the de-excitation process in liquid hydrogen can be described in terms of a collision between two molecules, neither of which is significantly perturbed because of collisions with other molecules. This is called the isolated binary collision, or IBC, assumption. According to a criterion for its validity established by $R_{\text {. }}$ Zwanzig in a theory of vibrational relaxation rates in liquids (Ref。38), the IBC assumption is valid only if the frequency of vibration, $v$, is much 
higher than $x$, the average number of collisions experienced by a molecule per second:

$$
x<<
$$

This criterion can be thought of as a requirement that struck molecules have time to "forget" before being struck again. Although Zwanzig concluded that the IBC assumption does not hold in liquids, there are two factors which tend to make liquid hydrogen an exception. One of these, of course, is the low collision frequency associated with liquid-hydrogen temperatures. But more important is the relative "stiffness" of the hydrogen molecule, which results in vibration frequencies that are much higher than those of other molecules.

If $\mathrm{H}_{2}$ molecules can be regarded as "hard spheres" which do not participate in many-body collisions, then the number of collisions experienced by one molecule per second is (Ref。8, p.631)

$$
x=\sqrt{2} \pi \sigma^{2} \text { s n, }
$$

where $\sigma$ is the effective molecular diameter, $s$ is the mean velocity and $\mathrm{n}$ is the number of molecules per $\mathrm{cm}^{3}$. From Eqs. 5-10 and 5-18, it is found that $\sigma$ is roughly $3 \times 10^{-18} \mathrm{~cm}$ (actualiy the center-of-mass separation at which the repulsive force commences). For $20^{\circ} \mathrm{K}, \mathrm{s}$ is about $4 \times 10^{4} \mathrm{~cm} / \mathrm{sec}_{\text {, }}$ and for liquid hydrogen $\mathrm{n}=2.11 \times 10^{22}$ molecules per $\mathrm{cm}^{3}$. With these values, $\mathrm{Eq} .5-35$ gives $x=3.4 \times 10^{12} \mathrm{sec}^{-1}$. 
The value of $x$ obtained above applies rigorously only to the "hard sphere" case, where collisions are of negligible duration, and it would lose its significance if a typical collision lasted long enough for a third molecule to become involved. Whether 3-body collisions are actually predominant can be determined by comparing the collision duration with $1 / x$, the average time between 2 -molecule collisions. The maximum collision duration is obtained by dividing twice the maximum "penetration" of the potential barrier given by Eq. 5-10 by the average velocity. From Eqs. 5-10 and 5-18, it is found that, for molecules with energles corresponding to liquid-hydrogen temperature and head-on $(b=0)$ collisions, the maximum "classical penetration" is about $7 \times 10^{-10} \mathrm{~cm}$. The average velocity over the collision is estimated, rather grossly, to be $2 \times 10^{4} \mathrm{~cm} / \mathrm{sec}_{\text {. Hence, }}$ the maximum collision duration for the most probable energy in liquid hydrogen is of the order of $7 \times 10^{-14}$ seconds. The average time between collisions, $1 / x$, is $3 \times 10^{-13}$ seconds. Although the collision duration is shorter only by a factor of 4 for head-on collisions, it should be much less for a typical glancing collision. For energies higher up on the distribution than the most probable energy, the same kind of results should be obtained, since the penetration distance does not increase as fast as the veolcity. In regard to interactions that are close enough to cause superelastic scattering, It is concluded that 2-molecule collisions are predominant. 
The IBC assumption can now be tested by comparing the 2-molecule collision frequency with the vibration frequency. The latter is given by (Ref. 34, p.62)

$$
v=(1 / 2 \pi) \sqrt{\rho / \mu},
$$

where $\mu$ is the reduced mass, and $f$ the force constant or "stretching factor." For $\mathrm{H}_{2}, f=5.448 \times 10^{5}$ dynes/cm (Ref.20) and $\mu=0.83 \times 10^{-24}$ grams. Then, Eq. $5-35$ gives $v=1.29 \times 10^{14} \mathrm{sec}^{-1}$, and $\mathrm{Eq} .5-34$ is satisfied. Hence, the IBC assumption should be good for any vibrational level of $\mathrm{H}_{2}$.

5.5 Need for Superelastic Cross Sections in Prediction of Propellant Heating

Even though the IBC assumption appears to be theoretically sound in the case of liquid hydrogen, it is still possible that the rate of vibrational relaxation is affected by a constant perturbation of electronic orbits through long-range forces in the liquid environment. Therefore, the conclusion that the relaxation time for the first level is of the order of 100 minutes must be regarded as tainted with contingency, even if the extrapolation to liquid-hydrogen temperature holds. Moreover, no information is avallable on the relaxation rates of higher vibrational levels. In order to establish the role of molecular excitation in definitely the propellant heating problem, both theoretical and experimental data will be needed on 
low-energy superelastic collision cross sections for $\mathrm{H}_{2}$ molecules excited to various levels and subject to perturbation by liquid forces. The methods used to obtain such data might also be used to provide information on recombination rates. 
VI. INFRARED -RAY EMISSION AND ABSORPTION

\subsection{Emission and Absorption Probabilities}

The de-excitation of $\mathrm{H}_{2}$ molecules may occur by electromagnetic emission as well as by superelastic scattering. Although the ultimate translation of stored energy into thermal effects depends upon the rate of superelastic scattering (including recombination), the competing process of infrared emission could be the determining factor in the diffusion of energy through the liquid. If the transmission of radiative energy to the walls of the system were more rapld than the expulsion of the irradiated portion of the liquid, then the energy initially stored in excitation would go into wall-heating through lattice excitations (which are absent in the liquid, assuming it is gas-like), regardless of the inefficiency of the $\mathrm{H}_{2}$ superelastic process。

Fortunately, the infrared rays emitted by excited molecules have just the right energy to be easliy reabsorbed by neighboring molecules in the liquid. This process effectively reproduces the original excited state (with a completely negligible transfer of momentum) and, therefore, delays the transfer of energy to the walls. It is found, in fact, that a high probability of emission implies a high probability of absorption, so that the migration of electromagnetic energy through the liquid could be slow even for short lifetimes against emission. Experimental measurements of radiative energy transfer have been made for organic liquids (Ref.. 39). Although no 
data of this type are available for liquid hydrogen, it should be possible to estimate emission and absorption - and, hence, transmission - rates indirectly by an analysis of basic data in terms of electromagnetic theory.

The process of electromagnetic emission depends on a displacement of the center of electronic charge of a molecule with respect to the center of mass. In a homopolar molecule such as $\mathrm{H}_{2}$, the electric dipole moment is zero in the absence of perturbing forces, and emission (or absorption) can only occur, with low probability, by virture of the small quadrupole moment. Considering that for the asymmetric $\mathrm{HCl}$ molecule the lifetime against vibrational de-excitation by emission (Ref. 40) is of the order of $10^{-2}$ seconds (which is long compared to the lifetimes of electronic states), it would not be surprising if the lifetime of the unperturbed $\mathrm{H}_{2}$ molecule against radiation proved to be extremely long.

The probabilities per unit time for various types of radiative transitions in unperturbed $\mathrm{H}_{2}$ molecules have been calculated as described in Appendix C. The method is based upon Maxwell's equations, which define the classical electromagnetic laws, and on Bohr's correspondence principle, which states that in a large assembly of excited systems the average rate of quantum emission is equal to the average rate of continuous emission predicted on the classical basis. The results are shown in Table III. The spectroscopic notation for a transition from state $b$ to state a is given, where the vibration numbers corresponding to $b$ and $a$, respectively, are $v^{b}$ and 
$v$ and where the rotation numbers corresponding to $b$ and $a$, respectively, are $J '$ and $J$. The wave number and wavelength of the emitted infrared radiation are $\sigma$ and $\lambda$, respectively. The average lifetime against the particular transition is the reciprocal of $A(b, a)$, the probability of emission per unit time. It is seen that for the unperturbed molecule ( $e_{\circ} g_{\circ}$, in gas at low pressure) the lifetimes against emission are greater than $10^{6}$ seconds

$A(b, a)$ is referred to as the "Einstein coefficient for spontaneous transition" (for the type of moment involved), and it gives the probability per unit time of spontaneous transition from state $b$ to state a. A related Einstein coefficient, called $B(a, b)$, represents the effectiveness of a radiation field in causing transitions, $i_{0} e_{0}$, in bringing the system from a lower to a higher level or vice versa. $B(a, b)$ therefore determines the coefficient of absorption for infrared rays. It is defined so that the number of radiationstimulated transitions from state a to state $b$ per unit time and per unit volume is given by

$$
n=N(a) B(a, b) p(\sigma),
$$

where $N(a)$ is the number of a-states per unit volume, $\sigma$ the wave number corresponding to the transition, and $\rho(\sigma)$ the spectral energy density of the field which induces the transition. The cgs units of $\rho(\sigma)$ are erg $/ \mathrm{cm}^{3}$ per $\mathrm{cm}^{-1}$ or erg $/ \mathrm{cm}^{2}$. The cgs units of $A(b, a)$ and $B(a, b)$ are $\mathrm{sec}^{-1}$ and $\mathrm{cm}^{2} / \mathrm{erg}-\mathrm{sec}$, respectively. The Einstein 
coefficients, $A(b, a)$ and $B(a, b)$, can be related by the principle of detalled balance (Ref. 41, p. 696), which states that in statistical equilibrium the rates of an elementary process and its inverse are equal. Thus, although each coefficlent has the same value whether or not equilibrium exists, the correct connection between the two is given by the statement of equilibrium,

$$
N(a) B(a, b) p(\sigma)=N(b) A(b, a)+N(b) B(b, a) p(\sigma),
$$

where $b$ is the higher state, so that $A(a, b)=0$. The number of states $a$ and $b$ per unit volume is given by the Maxwell-Boltzmann relation,

$$
N(a)=N g(a) \exp \left[-E_{a} / k T\right] \text {, }
$$

where $N$ is the number of molecules per unit volume and where $g(a)$ is the statistical weight of $a$, accounting for possible degeneracy (Ref. 41, p. 17). In the absence of an external field, $g(a)$ equals $2 J_{\mathrm{a}}+1$, the number of possible magnetic alignments. The equilibrium radiation energy-density distribution is given by Planck's formula (Ref。37, p.325):

$$
\rho(\sigma)=8 \pi h c \sigma^{3} /[\exp (h c \sigma / k T)-1]
$$

Substituting Eqs. 6-3 and 6-4 into Eq. 6-2 and observing that the result must hold for all $T$, one obtains

$$
A(b, a)=8 \pi h c \sigma^{3} B(b, a)
$$


Table III

Emission and Absorption Coefficients for Unperturbed $\mathrm{H}_{2}$ Molecules

\begin{tabular}{|c|c|c|c|c|c|c|c|c|c|}
\hline \multirow[b]{2}{*}{ Band } & \multirow[b]{2}{*}{ Line } & \multicolumn{2}{|c|}{ State b } & \multicolumn{2}{|c|}{ State a } & \multirow{2}{*}{$\begin{array}{c}\sigma \\
\left(\mathrm{cm}^{-1}\right)\end{array}$} & \multirow{2}{*}{$\begin{array}{c}\lambda \\
\text { (Angstroms) }\end{array}$} & \multirow{2}{*}{$\begin{array}{l}A(b, a) \\
\left(\sec ^{-1}\right)\end{array}$} & \multirow{2}{*}{$\begin{array}{c}\mathrm{B}(\mathrm{a}, \mathrm{b}) \\
\left(\mathrm{cm}^{2} / \mathrm{erg}-\mathrm{sec}\right)\end{array}$} \\
\hline & & $v^{\prime}$ & J & $\mathrm{v}$ & $\mathrm{J}$ & & & & \\
\hline$I=0$ & $s(0)$ & 1 & 2 & 0 & 0 & 4498 & 22228 & $0.804 \times 10^{-7}$ & $8.84 \times 10^{-4}$ \\
\hline $1=0$ & $S(1)$ & 1 & 3 & 0 & 1 & 4713 & 21213 & $1.30 \times 10^{-7}$ & $5.81 \times 10^{-4}$ \\
\hline $1-0$ & $Q(1)$ & 1 & 1 & 0 & 1 & 4155 & 24060 & $1.08 \times 10^{-7}$ & $3.02 \times 10^{-4}$ \\
\hline$I=0$ & $0(2)$ & 1 & 0 & 0 & 2 & 3807 & 26263 & $1.75 \times 10^{-7}$ & $1.27 \times 10^{-4}$ \\
\hline $2-0$ & $s(0)$ & 2 & 2 & 0 & 0 & 8403 & 11898 & $2.58 \times 10^{-7}$ & $4.35 \times 10^{-4}$ \\
\hline $3-0$ & $s(0)$ & 3 & 2 & 0 & 0 & 12080 & 8276.2 & 1. $2 \times 10^{-7}$ & $0.65 \times 10^{-4}$ \\
\hline
\end{tabular}


as wel1 as

$$
g(a) B(a, b)=g(b) B(b, a) ; g(a)=2 J_{a}+1 \text {. }
$$

Thus, $B(a, b)$ and $A(b, a)$ are related through Eqs, 6-5 and 6-6. Table III gives the values of $\mathrm{B}(\mathrm{a}, \mathrm{b})$ for unperturbed $\mathrm{H}_{2}$ molecules.

Since the electronic distribution of an $\mathrm{H}_{2}$ molecule in liquid hydrogen may be distorted both by close interactions with other molecules and by the integrated effect of long-range molecular forces in the liquid, the values of $A(b, a)$ and $B(a, b)$ for unperturbed molecules would not be expected to apply in the liquid. That perturbations have a much greater effect on lifetimes against emission than on "superelastic" relaxation times is empirically suggested by the emission lifetimes and relaxation times for asymmetric molecules, regarded as representative of $\mathrm{H}_{2}$ molecules subject to large distor tions. For gas at low density and at room temperature, the emission lifetime and relaxation time of $\mathrm{H}_{2}$ are of the order of 107 seconds (Table III) and $10^{-4}$ atmosphere-seconds (from Eq. 5-33), respectively。 For asymmetric molecules (and presumably for highly perturbed $\mathrm{H}_{2}$ molecules), the corresponding times are of the order of $10^{-2}$ seconds (Ref. 40) and $10^{-6}$ atmosphere-seconds (Ref. 31 and the references cited therein). It is seen that, provided a comparison of this sort is meaningful, perturbations influence emission lifetimes about 107 times as strongly as "superelastic" relaxation times. Therefore, the effects of liquid perturbations on the emission and absorption coefficients may be decisive. Although lack of information on the 
charge distortion prevents calculation of $A(b, a)$ and $B(a, b)$ by the method of Appendix $C$, estimates of $B(a, b)$ and, hence $A(b, a)$, can be made from infrared absorption data.

6.2 Significance of Experimental Infrared Absorption Spectra for the Liquid

The Einstein coefficient for absorption, $B(a, b)$, can be obtained experimentally by the examination of the absorption spectrum produced by the infrared irradiation of a small sample of material. From Eq. $6-1$, It follows that the energy absorption per unit area and per unit time of a beam of energy-intensity, $I_{0}(\sigma)$, is given by

$$
\int_{0}^{\infty} I_{0}(" \sigma) d^{\prime \prime}-\int_{0}^{\infty} I\left({ }^{\prime \prime}, \Delta x\right) d{ }^{\prime \prime} \equiv h v[N(a) B(a, b) \rho(\sigma)] \Delta x,
$$

where $\Delta x$ is the sample thickness and $I(\sigma, \Delta x)$ is the transmitted energy intensity. Since the incident radiation travels at velocity $c$, the energy-intensity is related to the energy density by

$$
I_{0}(\sigma)=c \rho(\sigma) \text {. }
$$

Therefore,

$$
\int_{0}^{\infty} I_{0}\left(\sigma^{\prime \prime}\right) d \sigma-\int_{0}^{\infty} I\left(\sigma^{\prime \prime}, \Delta x\right) d \sigma=I_{0}(\sigma) \frac{h v}{c} B(a, b) N(a) \Delta x_{0}
$$

In writing the integrals on the lefthand side of Eqs. 6-7 and 6-9, It is implicitly assumed that the "cross-section" for absorption is proportional to the Dirac function, $\delta\left(\sigma^{i}-\sigma\right)$.

Actualiy, an absorption or an emission line has a finite width which is due to the finite lifetimes of the initial and final levels 
(Ref。 42, p。82). According to quantum theory, the probability that the wave number of the emitted or absorbed photon will be displaced a "distance" $\sigma$ " from the values $(\sigma)$ predicted by Bohr's relation between energy change and wavelength is

$$
p\left(\sigma^{\prime}\right)=\frac{1}{\pi} \frac{1 / \sigma^{\prime} 0}{1+\left(\sigma^{\prime} / \sigma_{0}^{\prime}\right)^{2}} ;
$$

$\sigma^{\prime}$ is the half-width of the line and can be determined empirically by relating Eq. 6-10 to the observed integrated absorption. It is necessary to allow for the shape of the line in two situations: (1) if an experiment beam, which is initially almost independent of $\sigma^{\prime}$, penetrates a large thickness, a "hole" in the distribution will be created in the neighborhood of $\sigma$; and (2) if the penetrating photons are due to emission in the absorbing medium, the initial intensity distribution, $I_{0}(\sigma)$, will be peaked with the same shape as the absorption probability (Eq,6-10). In order to deal with such cases, it is necessary to define the coefficient $b\left(a, b, \sigma^{\prime}\right)$ so that the number of absorptions per unit volume, per unit time, and per unit wave number is given in analogy to Eq, 6-1 as

$$
n\left(\sigma^{\prime}\right)=N(a) b\left(a, b, \sigma^{\prime}\right) \rho\left(\sigma^{\prime}\right)
$$

The cgs units of $b\left(a, b, \sigma^{\prime}\right)$ are $\mathrm{cm} 3 /$ erg asecond. From the definitive Eq. $6-11$, it is clear that $b\left(a, b, \sigma^{\prime}\right)$ must have the same kind of distribution in wave number as $p\left(\sigma^{\prime}\right)$ in $E q .6-10$. This means that

$$
b\left(a, b, \sigma^{\prime}\right)=\left[\frac{1}{1+\left(\sigma^{\prime} / \sigma^{\prime} o\right)^{2}}\right] b(a, b, o) \text {. }
$$


Substituting Eq, 6-12 in Eq,6-11 and integrating over $\sigma^{\prime}$ with the assumption that $\rho(\sigma)$ does not vary over the line width gives

$$
n=\int n\left(\sigma^{\prime}\right) d \sigma^{\prime}=\rho(\sigma) N(a) \pi \sigma_{0}^{\prime} b(a, b, 0) \text {. }
$$

Since, the assumption of a Diracotype function for $\rho\left(\sigma^{8}\right)$ has, in effect, been made in $\mathrm{Eq}$ 。6-13, the result should be the same as in Eq. 6-1. Hence, the Einstein coefficient $B(a, b)$, which is obtained experimentaliy if $I_{0}\left(\sigma^{\prime}\right)$ is independent of $\sigma^{\prime}$ and if $\Delta x$ is small, is related to $b(a, b, \sigma)$ by

$$
b(a, b, c)=B(a, b) / \pi \sigma a
$$

From Eq. 6-11, the amount of energy absorption per unit volume, per unit time, and per unit wave number is

$$
h v n\left(\sigma^{3}\right)=h v N(a) b\left(a, b, \sigma^{1}\right) p\left(\sigma^{0}\right)=I\left(\sigma^{1}\right) \frac{h v}{c} N(a) b\left(a, b, \sigma^{1}\right),(\sigma-15)
$$

Then the change in intensity associated with an increment $\Delta x$ (the energy absorbed in the increment per unit area, per unit time, and per unit wave number) is

$$
-\Delta I\left(\sigma^{0}, x\right)=I\left(\sigma^{0}, x\right) \frac{h v}{c} b\left(a, b, \sigma^{0}\right) \Delta x_{0}
$$

Therefore, the spectral absorption is described by or, using Eqs. 6-12 and 6-14,

$$
\begin{aligned}
& I\left(\sigma^{\prime}, x\right)=I\left(\sigma^{\prime}, 0\right) \exp \left\{-\left[\frac{h v}{c} N(a) b\left(a, b, \sigma^{3}\right)\right] \times\right. \\
& \text { Eqs. } 6-12 \text { and } 6-14,
\end{aligned}
$$




$$
I\left(\sigma^{\prime}, x\right)=I\left(\sigma^{\prime}, 0\right) \exp \left\{-\left[\frac{h v}{c}\left(\frac{1}{\pi} \frac{1 / \sigma_{0}}{1+\left(\sigma^{\prime} / \sigma_{0}^{\prime}\right)^{2}}\right) N(a) B(a, b)\right] x\right\} \text {. }
$$

The integrated absorption or transmission is obtained in general by integrating Eq. 6-18 over $\sigma^{\prime}$. However, in the analysis of most absorption experiments, Eq. 6-9 is adequate, since the beam depletion is usually small. In the case of self-attenuation, where the distribution in wave number of the emitted photons has the same shape as the absorption probability, the effective absorption coefficient should be approximately equal to the coefficient of $x$ in Eq,6-18 with $\sigma^{\prime}=0$. Thus, the effective relaxation length for self-absorption is approximately (underestimating)

$$
\ell=\frac{\pi}{h \sigma} \quad \frac{\sigma_{0}^{\prime}}{\mathrm{N}(\mathrm{a}) \mathrm{B}(\mathrm{a}, \mathrm{b})}
$$

It is seen that a description of inf rared-ray attenuation in a medium can be based on "thin sample" measurements of the integrated absorption and half-width of the line, provided the experimental resolution is sufficient.

\subsection{Emission and Self-Absorption in Liquid Hydrogen}

In hydrogen gas at high pressures and in liquid hydrogen, infrared transitions are predominantly due, not to the quadrupole moment of the molecule but, rather, to a dipole moment which is induced by the proximity of other molecules. A treatment of this effect for densities low enough that only two or three molecules interact at 
once has been given in a series of papers by Van Kranendonk (Refis. 43, 45)。 In his first paper, Van Kranendonk expands the absorption coefficient in powers of density and develops a general formalism for the density and temperature-dependence of induced infrared transitions. This paper is followed by one in which the binary (2-molecule) absorption coeficients of the fundamental ( $v: 0$, $\left.v^{\prime}=1\right)$ band of hydrogen are derived. In his third paper, Van Kranendonk calculates the ternary (3-molecule) absorption coefficient for pure hydrogen and mixtures. His results are in general accord with recent infrared absorption measurements on hydrogen gas at high pressures (Ref。46). It is found that the process of charge distortion may be separated into two effects: (1) a long-range action due to the quadrupole fields of other molecules and (2) so-called overlap collisions in which the molecule comes into close collision with one or more of 1 ts neighbors. These processes are active in the liquid as well as the gas, but Van Kranendonk's treatment does not apply because of the high densities involved.

Fortunately, a Canadian group has measured the infrared absorp. tion of liquid hydrogen (Refs. 47, 48). Using a low attenuation sample, they obtained an intensity curve as a function of wave number for solid, liquid, and gaseous hydrogen - all at approximately the same density and at $11^{\circ} \mathrm{K}, 18^{\circ} \mathrm{K}$, and $298 \mathrm{~K}$, respectively (Fig。6)。 Comparison of the three curves shows how the absorption peaks broaden 
with increasing temperature. This is due to the shorter state lifetimes associated with more intimate contact between molecules. Since Eq. 6-18, which describes the attenuation of infrared rays through a material, applies regardless of the origin of the moment which causes the absorption, it should be possible to determine $B(a, b)$ from the peak corresponding to a transition between states $a$ and $b$. The experimental data are given in terms of $\log _{10}\left(I_{0} / I\right)$, where $I_{0}$ is the same as the $I\left(\sigma^{\prime}, 0\right)$ of Eq. 6-18. From Eq. 6-18,

$$
\log _{10}\left(I_{0} / I\right)=\frac{1}{2.303}\left[h \sigma \frac{1}{\pi} \frac{1 / \sigma_{0}}{1+\left(\sigma 1 / \sigma_{0}\right)^{2}} N(a) B(a, b)\right] \Delta x \text {. }
$$

Multiplying each side of Eq. $6-20$ by do' and integrating over all $\sigma^{\prime}$ gives

$$
\int_{-\infty}^{\infty} \log _{10}\left(I_{0} / I\right) d \sigma^{\prime}=\frac{1}{2.303}[h \sigma N(a) B(a, b)] \Delta x
$$

and

$$
B(a, b)=2.303 \int_{-\infty}^{\infty} \log _{10}\left(I_{0} / I\right) d \sigma^{\prime} / h \sigma N(a) \Delta x .
$$

Thus, the Einstein coefficient is proportional to the area under the appropriate peak in Figure 6 . It should be noted that to the right of each sharp peak in Figure 6 lies a poorly resolved broad peak. In each case these correspond to quadrupole induced moments and close collisions, respectively. The sharp peaks are supposedly at the same location as for the free molecule. The broad peaks are displaced because of the effects of collision between molecules. 


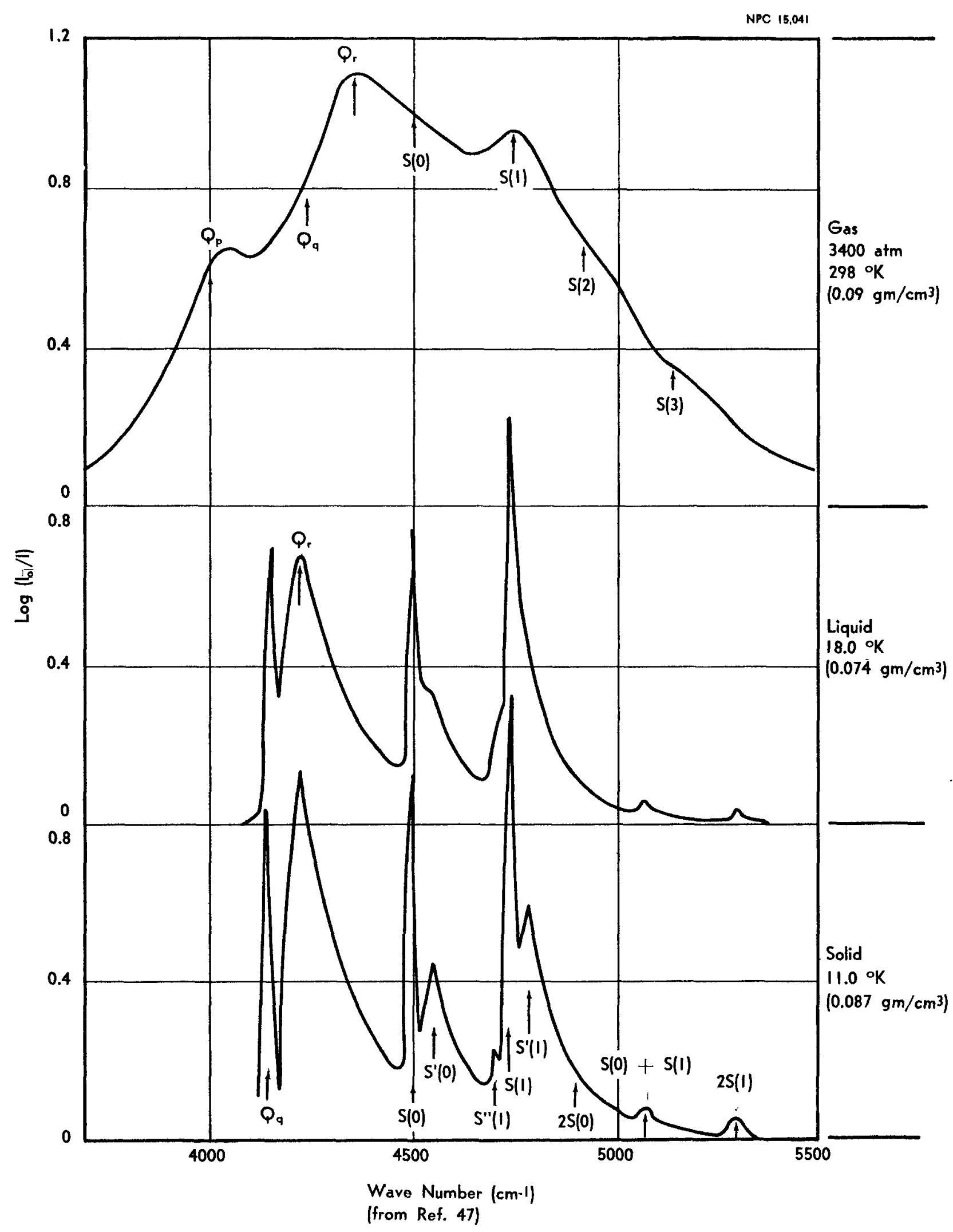

Figure 6. Infrared Absorption Spectra for Dense Hydrogen 
Since a given molecule is subject to each type of interaction for a certain proportion of the time, the probability of the particular transition is obtained by evaluating Eq. 6-22 for each peak and adding the results.

The liquid infrared absorption measurements were performed with normal hydrogen, which is a mixture of $25 \%$ parahydrogen and $75 \%$ orthohydrogen. This means that one-fourth of the molecules were initially in the rotational state $J=0$ and three fourths were initially in the rotational state $J=1$. The experimenters identified the sharp peaks near $4500 \mathrm{~cm}^{-1}$ and $4700 \mathrm{~cm}^{-1}$ with the $\mathrm{S}(0)$ and $\mathrm{S}(\mathrm{I})$ lines, respectively (see Table III). Although the peak in the neighborhood of $4150 \mathrm{~cm}^{-1}$ was only identified as a "Q component" (the $Q(1)$ and $Q(2)$ lines being separated only by $12 \mathrm{~cm}^{-1}$ ), it is reasonable to associate it with the $Q(1)$ line, since even at ordinary temperatures the $J=2$ level is relatively unpopulated. Under this assumption, Eq. 6-22 can be used to calculate $B(a, b)$ for the $Q(1)$ transition. In this case, $N(a)=3 / 4 N$, where $N$ is the number of molecules per unit volume. For the $S(0)$ and $S(1)$ lines, respectively, $N(s)=1 / 4 \mathrm{~N}$ and $\mathrm{N}(\mathrm{a})=3 / 4 \mathrm{~N}$. Defining $\mathrm{w}^{\prime}$ and $\mathrm{w}^{\prime \prime}$ as the integrals over corresponding sharp and broad peaks, respectively, the estimated integrals are given in Table IV. Labeling the corresponding coefficients as $B^{\prime}(a, b)$ and $B^{\prime \prime}(a, b)$, the total Einstein coefficient is given by

$$
B(a, b)=B^{\prime}(a, b)+B^{\prime \prime}(a, b) \text {. }
$$


Table IV gives the values of $B^{\prime}(a, b), B^{\prime \prime}(a, b)$, and $B(a, b)$ that were obtained with $\Delta x=0.84 \mathrm{~cm}_{2}$ the experimental sample thickness.

The values of $B(a, b)$ in Table IV are determined by the average charge distortion of hydrogen molecules in the liquid. If energy exchanges occurred only through electromagnetic transitions, it would be possible to define an Einstein coefficient for emission which was in effect the spontaneous transition coefficient $A(b, a)$ for a free molecule having the same average distorted charge distribution. Since at appreciable densities the transition may be brought about by superelastic scattering, in which the de-excitation energy goes into kinetic energy, the probability per unit time of transition from $b$ to $a$ is no longer equal to that for electromagnetic emission. However, it is st111 possible to define a probability of electromagnetic emission $A *(b, a)$ which can be calculated from $B(a, b)$ with Eq. 6-5. The reciprocal of $A^{*}(b, a)$ gives the average lifetime of state $b$, assuming that superelastic scattering does not occur. Table IV gives calculated values of $A *(b, a)$ for liquid hydrogen. The partial lifetimes against electromagnetic emission are seen to be of order $10^{1}$ seconds.

A final quantity of interest is the relaxation length, $b$, for self-absorption in the liquid. This is given by the approximate Eq. 6-19. It is less convenient to determine the half-width directly from graphical data than to express $b$ in terms of the peak values of $\log _{10}\left(I_{0} / I\right)$. From Eq. $6-20$, 


$$
\log \left[\frac{I_{0}}{I(0)}\right]=\frac{1}{2.303}[h \sigma N(a) \Delta x B(a, b)] \frac{1}{\pi \sigma^{\prime}} \text {. }
$$

Solving Eq.6-24 for $\sigma$ 'o and substituting in Eq. 6-19 gives

$$
b=\Delta x /\left\{2.303 \log _{10}\left[I_{0} / I(0)\right]\right\} \text {. }
$$

An effective selfabsorption length, $\bar{l}$, can be obtained by averaging over the two components of each line using $W^{\prime}$ and $W^{\prime \prime}$ for weights. Table IV gives $\bar{l}$ for normal liquid hydrogen, $1_{\circ} e_{\circ}$, for values of $N(a)$ for which three-fourths of the rotational states are $J=I$ and one-fourth is $J=O$. It is reasonable to assume that $\sigma^{\prime}$ and $B(a, b)$ do not depend strongly on the rotational states of the molecules in the liquid. Therefore, one can obtain the relaxation length for a given line in Ilquid hydrogen of arbitrary para- and ortho-hydrogen content by factoring appropriate values of $N(a)$ into the given values of $\bar{l}$.

The information in Table IV is limited to three lines of the "fundamental band" $\left(v=0, v^{\prime}=1\right)$. Although no data on the "overtone bands" ( $\left.=0, v^{\prime}>1\right)$ have been found for the liquid, measurements of the first overtone $\left(v=0, v^{\prime}=2\right)$ have been made for the gas at approximately Ilquid density and at $80^{\circ} \mathrm{K}$ (Ref. 49). With corrections for density and temperature differences between the gas and the liquid, the absorption data indicate values of the orders $\mathrm{B}(\mathrm{a}, \mathrm{b}) \sim 10^{1} \mathrm{~cm}^{2} / \mathrm{erg}-\mathrm{sec}, \mathrm{A}^{*}(\mathrm{~b}, \mathrm{a}) \sim 10^{-2} \mathrm{sec}^{-1}$, and $\mathrm{b} \sim 50 \mathrm{~cm}$ for the first overtone band $\left(8000-9000 \mathrm{~cm}^{-1}\right)$. 
Table IV

Emission and Absorption Coefficlents for H2 Molecules in Liquid Hydrogen and Mean Free Path of Emitted Radiation

\begin{tabular}{|c|c|c|c|c|c|c|c|c|c|c|c|}
\hline \multirow{2}{*}{ Line } & \multicolumn{2}{|c|}{ State a } & \multicolumn{2}{|c|}{ State b } & \multirow{2}{*}{$\begin{array}{c}W^{B} \\
\left(\mathrm{~cm}^{-1}\right)\end{array}$} & \multirow{2}{*}{$\begin{array}{c}W^{89} \\
\left(\mathrm{~cm}^{-1}\right)\end{array}$} & \multirow{2}{*}{$\begin{array}{c}\mathrm{B}^{\prime}(\mathrm{a}, \mathrm{b}) \\
\left(\mathrm{cm}^{2} / \mathrm{erg}-\mathrm{eec}\right)\end{array}$} & \multirow{2}{*}{$\begin{array}{c}B^{\prime \prime}(a, b) \\
\left(\mathrm{cm}^{2} / \mathrm{erg}-\mathrm{sec}\right)\end{array}$} & \multirow{2}{*}{$\begin{array}{c}\mathrm{B}(\mathrm{a}, \mathrm{b}) \\
\left(\mathrm{cm}^{2} / \mathrm{erg}-\mathrm{sec}\right)\end{array}$} & \multirow{2}{*}{$\begin{array}{l}A *(b, a) \\
\left(\sec ^{-1}\right)\end{array}$} & \multirow{2}{*}{$\begin{array}{c}\bar{l} \\
\text { (normal } \\
(\mathrm{cm})\end{array}$} \\
\hline & $\mathrm{v}$ & J & $\mathrm{v}^{8}$ & T & & & & & & & \\
\hline$s(0)$ & 0 & 0 & 1 & 2 & 27 & 35 & $4.486(2)$ & $5.724(2)$ & $10.210(2)$ & $0.929(-1)$ & .81 \\
\hline$S(1)$ & 0 & $I$ & $I$ & 3 & 36 & 45 & $1.903(2)$ & $2.335(2)$ & $4.238(2)$ & $0.951(-1)$ & .54 \\
\hline$Q(I)$ & 0 & 1 & 1 & 1 & 20 & 140 & $1.200(2)$ & $8.196(2)$ & $9.396(2)$ & $3.37(-1)$ & .51 \\
\hline
\end{tabular}


The values of $A(b, a)$ and $\bar{l}$ obtained for both the fundamental and the overtone bands show that if energy is transmitted through the liquid by infrared emission and absorption, the process is gradual. The product $\bar{l} A^{*}(b, a)$ may be regarded as the effective velocity of infrared-ray propagation in the direction of emission. Since $\bar{l} A^{*}(b, a)$ ranges from around $0.1 \mathrm{~cm} / \mathrm{sec}$ for the fundamental to about $0.5 \mathrm{~cm} / \mathrm{sec}$ for the overtone, it is seen that the rate of inf raredray-energy diffusion in the liquid is much less than $1 \mathrm{~cm} / \mathrm{sec}$.

Data on purely rotational transitions in liquid hydrogen are also avallable (Ref。24). The absorption half-widths of the $S(0)\left(355 \mathrm{~cm}^{-1}\right)$ and $S(1)\left(587 \mathrm{~cm}^{-1}\right)$ IInes of the $0-0$ band were found to be of the order $10^{-1} \mathrm{~cm}$. Since no separation into sharp and broad peaks was reported, it can be assumed that the emission iffetimes for purely rotational transitions are quite long.

From the standpoint of hydrogen propellant systems, the foregoing results mean that:

1. If energy partition by superelastic scattering proceeds slowly compared to the outflow of fluid from the system, the effect of infrared-ray emission will not reduce the expulsion of potential energy in the form of excited $\mathrm{H}_{2}$ states, provided the irradiated fiuid is expelied within several hundred seconds.

2. If energy partition by superelastic scattering is slow, the energy flux of inf rared rays trapped in the IIquid will be high relative to the radiation-energy flow into the liquid.

3. Therefore, one experimental method for determining the importance of delayed-energy partition would be to measure the infrared intensity in an irradiated sample. Because of the trapping of infrared rays in the liquid, it might be possible to perform spectral measurements well away from the nuclear radiation. 
VII. CONCLUSIONS

The conclusions of the present study are summarized below.

1. At least $43 \%$ of the energy deposited by radiation in liquid hydrogen is stored temporarily, mainly through the dissociation of $\mathrm{H}_{2}$ molecules. A large fraction of the remaining energy may be stored through inelastic collisions between energetic $\mathrm{H}$ atoms and molecules in the liquid.

2. For the flow rates presentiy envisioned, most of the $\mathrm{H}$ atoms produced by radiation $w 111$ be expelled for incident energy fluxes up to at least $1017 \mathrm{ev} / \mathrm{cm}^{2}-\mathrm{sec}$. Experimental results are consistent with predominant Hoatom expulsion at arbitrarily high radiation leyels.

3. Excited $\mathrm{H}_{2}$ molecules, which are apt to be formed in significant concentrations by recombinations and by inelastic scattering, may remain excited in the liquid for much longer than the 20 seconds or so required for the expulsion of the irradiated portion of the 11quid. Extrapolation of the theoretical relaxation time, while not conclusively valid, indicates that the Iifetime of the first vibrational level in the liquid is of the order of 100 minutes.

4. The lifetime of excited $\mathrm{H}_{2}$ states against emission in the Ilquid is of the order of seconds; however, the mean free path against absorption by re-excitation of the original type of $\mathrm{H}_{2}$ state is so small that the effective rate of infrared-ray diffusion is much less than $1 \mathrm{~cm} / \mathrm{sec}$. Hence, in the event that the partitioning of energy by superelastic scattering goes slowly then the walls of the liquid hydrogen tank wil1 not be heated significantly by infrared radiation. The storage of energy in the form of exclted $\mathrm{H}_{2}$ states can be verified experimentally by the observation of infrared rays trapped in the 11quid.

5. It is concluded that, insofar as radiation heating of the propellant has a signiflcant influence on the design of a nuclear-powered vehicle, further investigations into the rates of radiation-induced molecular reactions are warranted. Furthermore, any experimental data obtained on the radiation heating of liquid hydrogen should be interpreted in the light of possible delays in the equipartition of energy stored in radiation products. 


\section{Blank page}




\section{APPENDIX A}

\section{CALCULATION OF $\mathrm{H}_{3}$ IIFETIME}

The $\mathrm{H}_{3}$ complex is an unstable linear configuration (Ref。12)。 It holds together temporarliy because the energy of the system has been transferred from degrees of freedom corresponding to relative motion of the $\mathrm{H}$ atom and $\mathrm{H}_{2}$ molecule to internal degrees of freedom corresponding to vibration. There is a finite probability that the energy will again become concentrated in a mode similar to the original one, whereupon decomposition occurs.

An informative treatment of decomposition probabilities for unstable molecules has been given by Rosen (Ref.17), who applied his results to obtain the decomposition probability for the $\mathrm{HO}_{2}$ (H-0-0) quasi-molecule. Rosen's treatment is quite approximate in that a perturbation method is used and that certain terms are neglected. However, it is in no sense a "small vibration" treatment, as is evident from the fact that decomposition must be considered. The outlines of the procedure are given here.

Consider a linear triatomic complex composed of atom 1, atom 2, and atom 3 from left to rights and assume for the sake of definiteness that it was formed by the collision of atom 1 with a diatomic molecule composed of atoms 2 and 3. Let the masses of the atoms be $m_{1}, m_{2}$, and $m_{3}$, and let $r_{2}$ be the separation of the nuclei of atoms 2 and 3. Then the Schodinger equation for the nuclear motion may be written 
$\left.\begin{array}{c}\frac{-h^{2}}{8 \pi^{2}}\left[\frac{1}{\mu_{1}} \frac{\partial^{2}}{\partial r_{1}^{2}}+\frac{1}{\mu^{2}} \frac{\partial^{2}}{\partial r_{2}^{2}}-\frac{2}{m_{2}} \frac{\partial^{2}}{\partial r_{1} \partial r_{2}}\right]+v_{1}\left(r_{1}\right)+v_{2}\left(r_{2}\right)+v_{3}\left(r_{1}+r_{2}\right) \\ =E \psi,\end{array}\right\}_{(A-1)} \psi$

where $\mu_{1}=m_{1} m_{2} /\left(m_{1}+m_{2}\right), \mu_{2}=m_{2} m_{3} /\left(m_{2}+m_{3}\right)$, and $v_{1}, v_{2}$, and $v_{3}$ are the potential energies between atom pairs 1 and 2,2 and 3 , and $I$ and 3, respectively. In using the sum $V_{1}+V_{2}+V_{3}$ in $E q$. $A-1$, it is assumed that the interatomic potentials are strictly additive. Although in reality such potentials are only partialiy additive (Ref. 12), it can be assumed with reasonable accuracy that the potential energy of a group of atoms can be expressed as a sum of functions, each of which depends on the separation between a pair of atoms. In other words, the potentials can, hopefully, be added as in the case of conservative fields, provided the right functional forms are used. A sultable form is that proposed by Morse (Ref. 35) for diatomic molecules:

$$
V(r)=D e^{-2 a\left(r-r_{0}\right)}-2 D e^{-a\left(r-r_{0}\right)}
$$

The parameters $a$ and $D$ to be used for a pair of atoms in a many-atom problem are assumed to be the same as those for the corresponding diatomic molecule. This greatly simplifies matters, since the parameters for diatomic molecules can be obtained from spectroscopic data. 
In the solution of $\mathrm{Eq}$. A-1, Rosen's princlpal assumption is that the quantity,

$$
T \equiv\left(\frac{h^{2}}{4 \pi^{2} m_{2}} \frac{\partial^{2}}{\partial r_{1} \partial r_{2}}\right)+v_{3}
$$

Is small compared to the other terms, so that $T$ can be treated as a perturbation. Then it can be shown, in a manner equivalent (with a suitable redefinition of coordinates) to the derivation of a scattering cross section using the Born approximation, that the probability of dissociation per unit time is (Ref.34, p. 195 ff。)

$$
\left.\Gamma=\left(4 \pi^{2} / \mathrm{h}\right) \mid \iint \psi_{\mathrm{mn}}^{\mathrm{o}} \mathrm{T} \psi q_{\mathrm{s}}\right)\left.\mathrm{k} d \mathrm{r}_{1} \mathrm{dr} \mathrm{r}_{2}\right|^{2}
$$

The mean Iifetime of the complex, $T$, is the reciprocal of $\Gamma$. As in the case of the Born approximation, $\psi_{\mathrm{mn}}^{\circ}$ and $\psi(\mathrm{s}) \mathrm{k}$ are the solutions of the unperturbed system ( $1 . e_{0}, E q . A-I$ with $T=0$ ) before and after the transition. In this Instance, "before" refers to a non-dissocia. tive $\mathrm{H}_{3}$ state. The subscripts $m$ and $n$ correspond to discrete energy eigenvalues of the two equations into which the unperturbed form of $\mathrm{Eq} . \mathrm{A}=1$ separates:

$$
-\left(h^{2} / 8 \pi^{2} \mu_{1}\right) \frac{d^{2} \phi_{m}^{(1)}}{d r_{1}^{2}}+v_{1} \phi_{m}^{(I)}=E_{m}^{(I)} \phi_{m}^{(1)}
$$

and

$$
-\left(h^{2} / 8 \pi^{2} \mu_{2}\right) \frac{d^{2} \phi(2)}{d r_{2}^{2}}+v_{2} \phi_{n}^{(2)}=E_{n}^{(2)} \phi_{n}^{(2)}
$$


The solution of $\mathrm{Eq} . \mathrm{A}-1$ with $\mathrm{T}=0$ is

$$
\psi_{m n}^{\circ}=\phi_{m}^{(1)} \phi_{n}^{(2)} \text {, }
$$

with the energy elgenvalue being

$$
E_{m n}^{o}=E_{m}^{(1)}+E_{n}^{(2)} \text {. }
$$

The subscripts (s) and $k$ refer to an unperturbed solution of Eq. $A=1$ for which one of the Equations $A-5$ and $A-6$ has a continuous and the other a discrete solution.

Inspection of Eqs. $A-5$ and $A-6$ shows that $\phi_{m}^{(1)}$ and $\phi_{n}^{(2)}$ are essentially the solutions for diatomic molecules formed from particles $I$ and 2 and particles 2 and 3 , respectively. $E_{m}^{(1)}$ and $E_{n}^{(2)}$ are the corresponding energies of vibration or, in the case of a dissociative state, $E(s)$ corresponds to the total energy of a disintegrating molecule. If the perturbation, $T$, were completely negligible, a given pair of energies, $\mathrm{F}_{\mathrm{m}}^{(1)}$ and $\mathrm{E}_{\mathrm{n}}^{(2)}$, would be constant in time, so that no flow of energy to another mode of vibration could occur. Thus, a change of mode, such as is necessary for the formation or decomposio tion of a complex, is made possible only through the perturbation $T_{0}$ The solution of Eqs. A-5 and A-6 with the potential Eq. A-2 was f'lrst obtained by Morse (Ref. 35); Rosen remderived it in slightly different form. Rosen's results for a discrete state is (omitting particle subscripts)

$\phi_{n}=(-1)^{n} \Gamma(2 k-n) e^{-Z / 2} z^{k-\frac{3}{2}-n} \sum_{j=0}^{n}(-1)^{j}\left(\begin{array}{l}n \\ j\end{array}\right) \frac{z^{j}}{\Gamma(2 k-2 n-j)}$, 
where

$$
z=(4 \pi / a h)(2 \mu D)^{\frac{1}{2}} e^{-a\left(r-r_{0}\right)}
$$

and

$$
k=(2 \pi / a h)(2 \mu D)^{\frac{1}{2}}
$$

In the case of the continuous state, it is the asymptotic expression for $\phi(s)$ which is of interest, since decomposition corresponds to large separation. For an asymptotic relative kinetic energy $E_{\text {, this }}$ was found to be (keeping only terms of the largest order of magnitude)

$$
\phi(s) \sim 2 G \cos (\operatorname{sar}-s \lambda+\gamma),
$$

where

$$
\begin{aligned}
& s=(2 \pi / a h)(2 \mu E)^{\frac{1}{2}}, \\
& \left.\lambda=a r_{0}+\ln \left[4 \pi(2 \mu D)^{\frac{1}{2}} / a h\right)\right],
\end{aligned}
$$

and $G$ and $\gamma$ are such that

$$
G e^{i \gamma}=\Gamma(2 i s) / \Gamma\left(\frac{1}{2}-k+i s\right) \text {. }
$$

In order to normalize the wave function $\phi_{\mathrm{p}}$ to give unit probability for the space integral over a specific energy state, $p$, the condition,

$$
\lim _{\Delta \rightarrow 0}\left\{\frac{1}{\Delta} \int_{0}^{\infty}\left[\int_{p-\Delta / 2}^{p+\Delta / 2} \bar{\phi}_{p}(x) d p\right]\left[\int_{p-\Delta / 2}^{p+\Delta / 2} \phi_{p}(x) d p\right] d x\right\}=1
$$

must be met. This leads to the normalization constants $N_{n}$ and $N(s)$ : 
and

$$
N_{n}=\{[2 k-2 n-1] a /[n ! \Gamma(2 k-n)]\}^{\frac{1}{2}}
$$

$$
N(s)=\left\{(4 / h)\left[\mu \sinh 2 \pi s\left|\Gamma\left(\frac{1}{2}-k+\lambda s\right)\right|^{2} / a\right]^{\frac{1}{2}}\right\} \text {. }
$$

In order to simplify the evaluation of the dissociation probability, $\Gamma$, Rosen made one further assumption, namely, that the $V_{3}$ term in the expression for $T$ can be neglected. If this is the case, then

$$
\mathrm{T} \sim\left(\mathrm{h}^{2} / 4 \pi^{2} \mathrm{~m}_{2}\right) \gamma^{2} / \gamma_{\mathrm{r}}, \gamma_{2}
$$

The neglect of $V_{3}$ - the interaction between the outside atoms is supposed not to change the order of magnitude of the calculated $\Gamma$. If the absolute value of $V_{3}$ is small compared to $V_{1}$ and $V_{2}$, which are only approximately additive in any event, its neglect should not be important if the additivity assumption is permissible. Substituting the above expressions for $\psi_{\mathrm{m} n}^{\circ}, \psi^{\circ}(\mathrm{s}) \mathrm{k}$ and $\mathrm{T}$ into $\mathrm{Eq}$. A-4, Rosen obtained for the transition probability per unit time, essentially,

$$
\Gamma=\frac{h_{\mu} a z_{2}^{2} t\left(2 k_{2}-2 m-1\right)\left[\Gamma\left(k_{1}+\frac{1}{2}\right)\right]^{2} m ! \Gamma\left(2 k_{2}-m\right)}{m_{2}^{2} \Gamma\left(k_{1}+\frac{1}{2}-t\right) \Gamma\left(k_{1}+\frac{1}{2}+t\right) \Gamma\left(2 k_{2}-1\right) \prod_{j=0}^{\infty}\left[1+s^{2} /\left(k_{1}+\frac{1}{2}+j\right)^{2}\right]} \quad(A-20)
$$

where $k_{1}$ and $s$ are given by Eqs. $A-11$ and $A-13$, respectively, $p$ and $m$ are the quantum numbers of the non-dissociative state corresponding to motion between particles 1 and 2 and particles 2 and 3 , respectively, and $t=k_{1}-p-1 / 2$. The total energy before and 
after decomposition must be the same. This imposes the condition,

$$
\left(a_{1}^{2} / \mu_{1}\right)\left(k_{1}-p-\frac{1}{2}\right)^{2}+\left(a_{2} / \mu_{2}\right)\left(k_{2}-m-\frac{1}{2}\right)^{2}=\left(a_{1}^{2} / \mu_{1}\right) s^{2}+\left(a_{2}^{2} / \mu_{2}\right)\left(k_{2}-n-\frac{1}{2}\right)^{2},
$$

where $\mathrm{n}$ is the quantum number for a possible vibrational excited state of the residual $\mathrm{H}_{2}$ molecule. In the case of interest here (as in Rosen's case), $n=0$. Then $s^{2}$ for decomposition is the same as that for collisions and only those $m, p$ pairs are permissible quantum numbers which satisfy Eq. A-2 with $\mathrm{n}=0$. The values of $m$ and $p$ which satisfy Eq.A-21 need only be approximately integers. The reason for this is that the energy levels for permissible modes have finite widths. Thus, the likelihood of forming an $\mathrm{H}_{3}$ complex in the first place depends on how closely Eq. A-2l with the given value of $s^{2}$ corresponds to a pair of integers $m, p$, which almost satisfy Eq。A-21。 Rosen made a final approximation which holds only for small $\mathrm{s}^{2}$ :

$$
\prod_{j=0}^{\infty}\left[1+s^{2} /\left(k_{1}+\frac{1}{2}+j\right)^{2}\right] \approx 1
$$

Th1s applies for collision energies corresponding to those at room temperatures. However, for collisions in the ev range, the product may have a value as high as $10^{6}$, so that it must be evaluated rather laboriously.

Rosen applied his result (Eq.A-20) to obtain the lifetime of the $\mathrm{H}-0-0$ quasi-molecule for several pairs of quantum numbers $\left(m_{s} p\right)$ 
and for $\mathrm{s}^{2}$ corresponding to room-temperature collisions. For this complex and mean lifetime, $\tau=1 / \Gamma$ ranged from orders of $10^{-14} \mathrm{sec}$ to $10^{-8} \mathrm{sec}$.

For the present objectives, Rosen's method has been applied to the calculation of lifetimes of the $\mathrm{H}_{3}$ complex without modification, except for the evaluation of the product in Eq. A-22. The 2-atom potential parameters, $a$ and $D$, were taken from Morse's compliation (Ref. 35). In this case, $a_{1}=a_{2}=1.85 \times 10^{8} \mathrm{~cm}^{-1}$ and $D_{1}=D_{2}=$ $7.97 \times 10^{-12} \mathrm{ergs}$ (Morse's data having been converted from angstrom and wave-number units). Values of $\mathrm{s}^{2}$ corresponding to collision kinetic energies of $0.5,1,2,3$, and $4 \mathrm{ev}$ have been considered. The most likely quantum pairs $(m, p)$ for a given $s^{2}$ were chosen by solving Eq. A-2l for $p$ and plotting the results as a function of $m$ (Fig. A I). In the general case of a linear triatomic molecule, the plot is an ellipse; in the case of $\mathrm{H}_{3}$, it is circular, since the atoms are identical.

The lifetime $\tau_{m p}$ with respect to decomposition from the mode $m, p$ is given in Table II. It should be noted that there are also lifetimes with respect to transitions to other discrete states, say m',p'. Such processes compete with the decomposition transition. It is found that, if the system can go to anyone of several discrete or continuous states, $\Gamma_{m p}$ is largest where the transition $m \rightarrow m$ ' and $p \longrightarrow p$ ' correspond to the smallest changes in $m$ and $p$. It is for this reason that only $m, p$ pairs from the second quandrant 
NPC 15,042

$$
p=18 \pm \sqrt{182-\left[s^{2}+(18-m)^{2}\right]}
$$

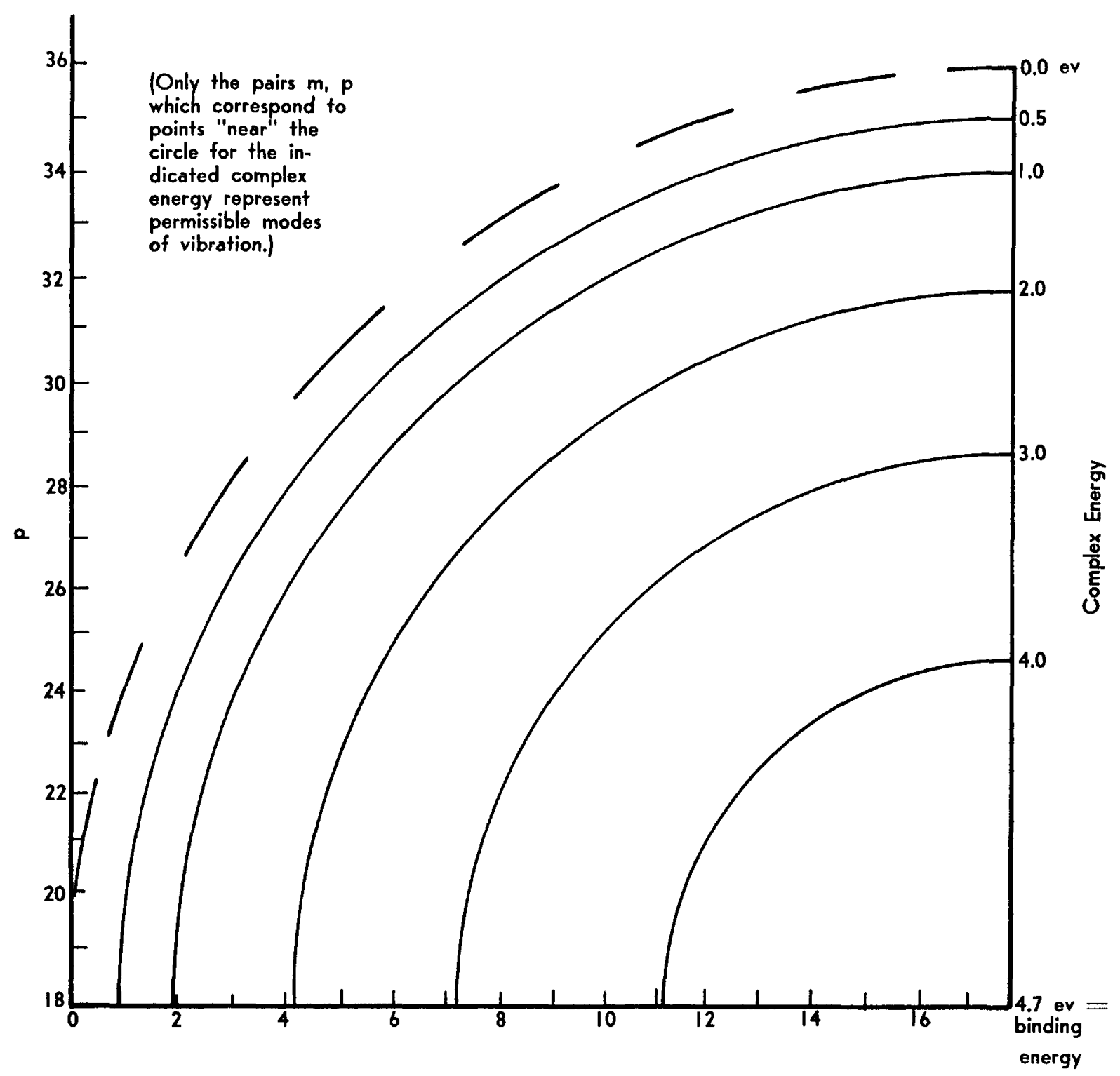

m

Figure A-1. Permissible Modes of $\mathrm{H}_{3}$ Vibration 
(small m, large $\mathrm{p}$ ) have been chosen; transitions from states corresponding to pairs outside this range would be much more apt to yield new discrete states than a decomposition state. 


\section{APPENDIX B}

THE SLOWING DOWN OF EXCITED H2 MOLECULES

The inelastic and superelastic cross sections can be related on a thermodynamic basis. Let $E_{0}$ be the kinetic energy of colliding molecules in the center of mass system, and let $E_{1}$ be the corres ponding energy after inelastlc scattering. If $w$ is the level excitation energy, then

$$
E_{0}=E_{1}+w_{0}
$$

For a system in equilibrium, using the notation of Section 5.3 ,

$$
\bar{n}_{1} d f_{10}=\bar{n}_{0} d f_{01}
$$

or

$$
\bar{n}_{1} \eta_{1}\left(v_{1}\right) d v_{1} v_{1} \sigma_{10}\left(v_{1}\right)=\bar{n}_{0} \eta\left(v_{0}\right) d v_{0} v_{0} \sigma_{01}\left(v_{0}\right)
$$

where $v_{I}$ and $v_{0}$ are the relative velocities corresponding to $E_{1}$ and Eo. Since equipartition of kinetic energy prevalis for both excited and un-excited states, $\eta(v) d v$ is given by Maxwell's equation,

$$
\eta(v) d v=4 \pi(m / 2 k T)^{3 / 2} v^{2} \exp \left(-m v^{2} / 2 k T\right) d v
$$

where $m$ is the reduced mass. It is seen that

$$
\eta(\mathrm{v}) \mathrm{dv} \propto \mathrm{v} \exp (-\mathrm{E} / \mathrm{kT}) \mathrm{dE} \text { 。 }
$$

From Eqs. $\mathrm{B}-3$ and $\mathrm{B}-5$, 


$$
\bar{n}_{1} \exp \left(-E_{1} / k T\right) v_{1}^{2} \sigma_{10}\left(v_{1}\right) d E_{1}=\bar{n}_{0} \exp \left(-E_{0} / k T\right) v_{0}^{2} \sigma_{01}\left(v_{0}\right) d E_{0}
$$

From Eq. $B-1, d E_{1}=d_{0}$, so that

$$
\sigma_{10}\left(v_{1}\right)=\left(\bar{n}_{0} / \bar{n}_{1}\right) \exp \left[-\left(E_{0}-E_{1}\right) / k T\right]\left(v_{0}^{2} / v_{1}^{2}\right) \sigma_{01}\left(v_{0}\right),
$$

where $\bar{n}_{0}$ and $\bar{n}_{1}$ are given by Eqs. 5-23 and 5-24, respectively. Since Eq. B-7 must hold for all values of $T$, it must be true that

$$
\sigma_{10}\left(v_{1}\right)=\left(v_{0}^{2} / v_{1}^{2}\right) \sigma_{01}\left(v_{0}\right)
$$

or, using Eq. B-I,

$$
\sigma_{10}\left(E_{1}\right)=\left[\left(E_{1}+w\right) / E_{1}\right] \sigma_{01}\left(E_{1}+w\right) .
$$

(Eq. B-9 is only exact in the absence of rotational transitions because of the stipulation in the derivation of $\bar{n}_{0}$ and $\bar{n}_{1}$ that only two "cells" were accessible.)

Using Eq. B-9, the superelastic scattering cross section for the de-excitation of the first vibrationally excited $\mathrm{H}_{2}$ level can easily be obtained from the crossosection curve shown in Figure 4. In this case, $w=h v=0.53 \mathrm{ev}$. It is seen that for a kinetic energy of around $1 \mathrm{ev}$ (which is about the maximum kinetic energy imparted to excited $\mathrm{H}_{2}$ molecules) the superelastic cross section is of the order of $4 \times 10^{-17} \mathrm{~cm}^{2}$. The effective "diameter" for momentum transfer should be about $3 \times 10^{-8} \mathrm{~cm}$ (based on the Morse potential used by Bauer and Salkoff; see Sec, 5.4). Therefore, the cross section for momentum transfer should be of the order of $3 \times 10^{-15} \mathrm{~cm}^{2}$. Since on the average 
half of the kinetic energy is lost in an elastic scatter and since the superelastic cross section is seen to fall off drastically with decreasing energy, there is little chance for de-excitation of the molecule in the 10 collisions or so required for effective "thermalization"。 
Blank page 


\section{APPENDIX $\mathrm{C}$ \\ CALCULATION OF ELECTROMAGNETIC TRANSITION PROBABILITIES}

Considered as a classical system, a vibrating or rotating $\mathrm{H}_{2}$ molecule should radiate continuously according to the solution of Maxwell's equations:

$$
\begin{aligned}
& \text { div } \underline{H}=0 \\
& \text { curl } \underline{E}+\frac{1}{C} \frac{\partial H}{\partial t}=0 \\
& \text { div } \underline{E}=4 \pi \rho \\
& \text { curl } \underline{H}=\frac{1}{C} \frac{\partial E}{\partial t}=4 \pi I
\end{aligned}
$$

(respectively representing non-existence of magnetic charge, Faraday ${ }^{\natural}$ law of electromagnetic induction, Coulomb's law of electrostatics, and Ampere's law for a current-induced fleld, together with Maxwel1's displacement current hypothesis), where $E$ and $H$ are the electric and magnetic fields, respectively, I is the current density field, and $\rho$ is the charge density field. The solution of Eq. C-I is obtained by defining the scalar and vector potentials $\varnothing$ and $\underline{A}_{\text {, so that }}$

and

$$
E=-\operatorname{grad} \varnothing-\frac{1}{C} \frac{\partial A}{\partial t}
$$

$$
\underline{E}=\operatorname{cur} 1 \stackrel{A}{0}
$$

Then, 
and

$$
\begin{aligned}
& -\Delta \phi+\frac{I}{c^{2}} \frac{\partial^{2} \phi}{t^{2}}=4 \pi \rho, \\
& -\Delta A+\frac{1}{c^{2}} \frac{\partial^{2 A}}{\partial t^{2}}=4 \pi I .
\end{aligned}
$$

$$
\operatorname{div} \underline{A}+\frac{1}{C} \frac{\gamma \phi}{\gamma^{t}}=0
$$

and it follows that

$$
\varnothing(x, y, z, t)=\int \frac{\rho\left(x^{\prime}, y^{\prime}, z^{\prime}, t^{\prime}\right)}{R} d x^{\prime} d y^{\prime} d z^{\prime}
$$

and

$$
A(x, y, z, t)=\int \frac{I\left(x^{\prime}, y^{\prime}, z^{\prime}, t^{\prime}\right)}{R} d x^{\prime} d y^{\prime} d z^{\prime},
$$

where $\underline{R}=\underline{r}-\underline{r}^{\prime}, t^{\prime}=t-R / C$.

Equations $\mathrm{C}-4$ are the retarded potential formulae which show how a volume element at $r^{\prime}$ contributes to the charge density at $\underline{r}$ according to the value at $\underline{r}^{\prime}$ at an earlier time. Equation $\mathrm{C}-3$ demonstrates the propagation of the electromagnetic field as a wave. Calculation of the potential energy stored in the electric and magnetic fields and comparison of the result with a surface integral of the form $\int E X \underline{H} \mathrm{dA}$ (Ref. 50, $p .175$ ) show that the flow of energy at a particular point and at a given instant according to the classical theory is given by the Poynting vector:

$$
\underline{S}=\frac{C}{4 \pi}(\underline{E} X \underline{H}) \text {. }
$$

Assuming that $\underline{E}$ and $\underline{H}$ vary harmonically with time, the average flow of energy is given by 


$$
\underline{S}_{a v}=\frac{1}{4} \frac{C}{4 \pi}(\underline{E} \times \underline{\tilde{H}}+\underline{\widetilde{E}} \times \underline{H}),
$$

where $\underline{\underline{E}}$ and $\underline{\widetilde{H}}$ are the complex conjugates of $\underline{E}$ and $\underline{H}$, respectively。 The classical theory may be put in terms suitable for use in quantum theory (Ref。42, p。103) . In view of the assumption that $\rho$ and I vary harmonically with time, so that they are of the form

$$
\rho(x, y, z, t)=R_{e}\left[\rho(x, y, z) e^{2 \pi 1 v t}\right]
$$

and

$$
I(x, y, z, t)=R_{e}\left[I(x, y, z) e^{2 \pi i v t}\right],
$$

it follows from Eq. $\mathrm{C}-4$ that

$$
\phi(x, y, z, t)=e^{2 \pi i \nu t} \int \rho\left(x^{\prime}, y^{\prime}, z^{\prime}\right) \frac{e^{-1 k R}}{R} d v
$$

and

$$
\underline{A}(x, y, z, t)=e^{2 \pi 1 v t} \int I\left(x^{\prime}, y^{\prime}, z^{\prime}\right) \frac{e^{-1 k R}}{R} d v,
$$

where $k=2 \pi \% / c$ 。

Expansion of the factor $e^{-i k R} / R$ in Eq. C.8 in terms of $\omega$, the angle between $\underline{\underline{r}}^{\prime}$ and $\underline{\underline{r}}$ gives

$$
\frac{e^{-i k R}}{R}=\sum_{\lambda=0}\left(2 \lambda \text { 1) } \frac{\xi_{\lambda}(k r)}{1 r} \frac{\psi_{\lambda}\left(k r^{i}\right)}{k r^{i}} p_{\lambda}(\cos \omega)\right.
$$

where the $P_{\lambda}$ are Legendre polynomials and $\xi_{\lambda}$ and $\psi_{\lambda}$ are Bessel. functions: 


$$
\psi_{\lambda}\left(k r^{\prime}\right)=\sqrt{\frac{1}{2} \pi k r^{\prime}} J_{\lambda+\frac{1}{2}}\left(k r^{\prime}\right)
$$

and

$$
\xi_{\lambda}(k r)=\sqrt{\frac{1}{2} \pi k r}\left[J_{\lambda+\frac{1}{2}}(k r)+(-1)^{2} J_{-\lambda-\frac{1}{2}}(k r)\right] .
$$

Keeping only the leading terms in $\psi_{\lambda}$, one obtains

$$
\begin{aligned}
& \left.\begin{array}{rl}
\varnothing=\frac{e^{2 \pi 1 v t-1 k r}}{r}\left\{\int \rho P_{0} d v+1 k\left(1-\frac{1}{k r}\right) \int \rho r^{\prime} P_{1} d v-\frac{k^{2}}{3}\left[\left(1-\frac{3 i}{k r}\right) \int \rho r^{\prime}{ }^{2} P_{2} d v\right.\right. \\
\text { and } & \left.+\frac{1}{2} \int \rho r^{\prime}{ }^{2} P_{0} d v\right]+\ldots .
\end{array}\right\}
\end{aligned}
$$

$$
\begin{aligned}
& \underline{A}=\frac{e^{2 \pi i v t-i k r}}{r}\left\{\int I P_{0} d v+i k\left(1-\frac{1}{k r}\right) \int I r \cdot P_{1} d v-\frac{k^{2}}{3}\left[\left(1-\frac{31}{k r}\right) \int I r{ }^{2} P_{2} d v\right.\right. \\
& \left.+\frac{1}{2}\left[I r^{\prime}{ }^{2} P_{0} d v\right]+\cdots\right\} \cdot(C-11)
\end{aligned}
$$

Eq. C-II is simplified by introducing the shorthand notation.

$\underline{r}_{0}=$ unit vector in the direction of $\underline{\underline{r}}$

$\underline{P}=\int \rho \underline{r}$ 'dv (a "dipole moment of $\rho "$ " = vector $)$

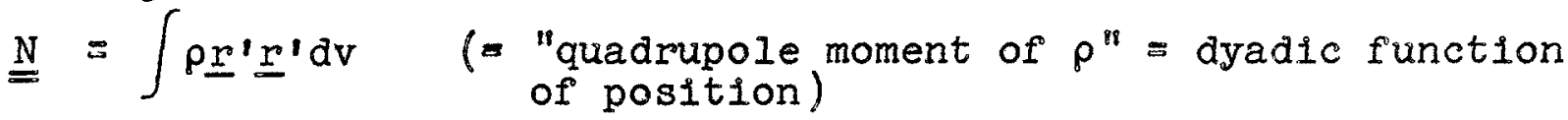

$N_{S}=\int p r \cdot{ }^{2} P_{0} d v$ (= invarient sum of the diagonal terms in $\underline{N}$ )

$\underline{M}=\frac{1}{2} \int \underline{r}^{\prime} \times I d v \quad\left(=\begin{array}{l}\text { magnetic moment of electric current } \\ \text { distribution }=\text { vector }) .\end{array}\right.$ 
From the equation of continuity (charge conservation)

$$
\operatorname{div} I=\frac{-I}{C} \frac{\partial p}{\partial t}=-1 \mathrm{kp} \text {. }
$$

From the finiteness of $C$, it follows that $\rho$ and $I$ vanish everywhere outside a finite sphere.

Then,

$$
i k \int \rho g d v=\int I \cdot \nabla g d v,
$$

where $g$ is any scalar, vector, or dyadic function of position.

Then

$$
\int \rho d v=0, \int I d v=i k \underline{P}, \int(\underline{I}+\underline{r} I) d v=i k \underline{\underline{N}} .
$$

Eq. C-II becomes

$$
\begin{aligned}
& \varnothing=\frac{e^{2 \pi i v t-i k r}}{r}\left\{1 k\left(1-\frac{1}{k r}\right) \underline{r}_{0} \cdot \underline{P}-\frac{1}{2} k^{2}\left[\underline{r}_{0} \cdot \underline{N} \cdot \underline{r}_{0}-\frac{1}{k r}\left(3 r_{0} \cdot N \cdot r_{0}-N_{S}\right)\right]\right\} \\
& \underline{A}=\frac{e^{2 \pi i v t-i k r}}{r}\left\{1 k P-i k\left(1-\frac{1}{k r}\right) \underline{r}_{0} \times \underline{M}-\frac{1}{2} k^{2}\left(1-\frac{1}{k r}\right) r_{0} \cdot \underline{N}\right\} . \quad(C-13)
\end{aligned}
$$

From Eqs. C-2, $\mathrm{C}-5$, and $\mathrm{C}-13$, the parts of $\underline{\mathrm{E}}$ and $\underline{\mathrm{H}}$ which involve $\underline{\mathrm{P}}$ to terms in $\mathrm{r}^{-1}$ and $\mathrm{r}^{-2}$ are

$\underline{E}(\underline{P})=\frac{e^{2 \pi i(v t-\sigma r)}}{r}\left\{\left(k^{2}-\frac{1 k}{r}\right)\left(\underline{F}-\underline{r}_{0} \underline{r}_{0}\right) \cdot \underline{p}+\frac{2 i k}{r} \underline{r}_{0} \underline{r}_{0} \cdot \underline{p}\right\}$
$\underline{H}(\underline{P})=\frac{e^{2 \pi i(v t-\sigma r)}}{r}\left(k^{2}-\frac{1 k}{r}\right) \underline{r}_{0} x \underline{P}$,

where $\mathscr{F}=\underline{11}+\underline{\mathcal{j}}+\underline{k k}$ ( $=$ unit dyadic). 
Then, from Eqs. C-6 and C-13, the part of the radiation due to the dipole moment $\underline{P}$ is, for large $r$ and according to the classical theory,

$$
\underline{S}_{a v}(\underline{p})=\frac{C}{8 \pi} \frac{k^{4}}{r^{2}}\left|\left(\underline{F}-\underline{r}_{0} \underline{r}_{0}\right) \cdot \underline{p}\right|^{2} \underline{r}_{0} .
$$

The parts of $\underline{E}$ and $\underline{H}$ involving the quadrupole moment $\underline{\underline{N}}$ to terms in $r^{-1}$ are

$$
E=\frac{i k^{3}}{2} \frac{e^{2 \pi i(\nu t-\sigma r)}}{r}\left(\underline{F}-\underline{r}_{0} \underline{r}_{0}\right) \cdot\left(\underline{N} \cdot \underline{r}_{0}\right)
$$

and

$$
\underline{H}=\frac{i k^{3}}{2} \frac{e^{2 \pi 1(v t-\sigma r)}}{r} \underline{r}_{0} \times\left(\underline{N} \cdot \underline{r}_{0}\right)
$$

and, from Eqs. C-6 and C-5, the part of the radiation due to the quadrupole moment alone is, according to the classical theory,

$$
\underline{s}_{a v}=\frac{c k^{6}}{32 \pi r^{2}}\left|\left(\underline{F}-\underline{r}_{0} \underline{r}_{0}\right) \cdot\left(\underline{N} \cdot \underline{r}_{0}\right)\right|^{2} \underline{r}_{0} \cdot
$$

Higher-order terms of $S_{\mathrm{av}}$ are generally held to be insignificant. In reality, of course, the emission of radiation by molecular systems Is quantum mechanical and occurs through discrete transitions. However, according to Bohr's correspondence principle, there is an asymptotic correspondence between classical and and quantum mechanics, so that the rate of quantum emission is on the average equal to the rate of continuous emission predicted by classical mechanics. 
Specifically, the radiation fleld accompanying a quantum transition is the same as that given by classical theory for a charge distribution whose moments are

$$
2 \operatorname{Re}\left\{(b|| a) e^{2 \pi 1 v t}\right\},
$$

where $(b|| a)$ is the matrix component of the kind of electric or magnetic field being considered. It follows that the probability per unit time of such a transition occurring is

$$
A(b, a)=\frac{I}{h \psi}\left(b\left|\int_{s} S_{a v} d A\right| a\right),
$$

where the integration is over a large surface enclosing the system and where $b$ and $a$ represent the initial and final states. The matrix element in $\mathrm{Eq}, \mathrm{C}-18$ is defined by

$$
(b|x| a)=\int \psi_{b}(\underline{r}) x(\underline{r}) \psi_{a}(\underline{r}) d \tau
$$

where $\psi_{b}$ and $\psi_{a}$ are the initial and final wave functions of the system (regarded as a collection of point charges) and $\underline{\underline{r}}$ represents all of the spatial variables. $A(b, a)$ is the Einstein coefficient for emission. The Einstein coefficient for absorption is related to $A(b, a)$ by $E q .6-5$ of the text.

A hydrogen molecule whose charge distribution is not perturbed by the proximity of other molecules has a vanishing dipole moment, owing to its symmetry of charge with respect to its center of mass. 
Therefore, $A(b, a)$ and $B(a, b)$ are both zero for dipole transitions. The quadrupole moment is of the form

$$
\underline{N}=\sum_{1} e_{1} \underline{r}_{1} \underline{r}_{1}
$$

where the $\underline{r}_{1}$ are the position vectors of the electrons and nuclei with respect to the center of mass. If the wave functions $\psi_{a}$ and $\psi_{\mathrm{b}}$ for the whole system are known, the Einstein coefficients can be calculated by application of Eqs. $\mathrm{C}-17, \mathrm{C}-18$, and $\mathrm{C}-20$. This has been done by James and Coolidge (Ref。 51) for the case of vibrationrotation transitions in the electronic ground state. They found that the coefficient for spontaneous transition reduces to

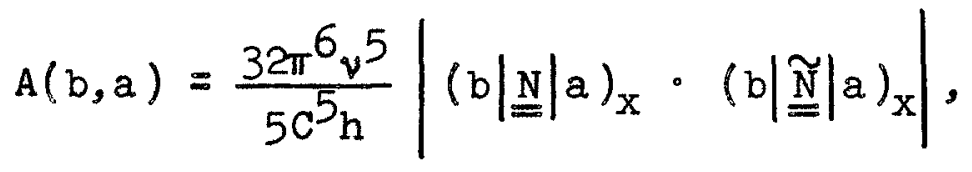

where

$$
(b|N| a)_{x} \equiv\left(b\left|N-\frac{1}{3}\{N\}_{s} g\right| a\right)_{0}
$$

The dot product between dyadics in $\mathrm{Eq}, \mathrm{C}-2 \mathrm{l}$ is so defined that

$$
\left(\underline{x}, \underline{y} \circ \underline{x}^{\prime} \underline{y}^{\prime}\right)=\left(\underline{x} \circ \underline{x}^{\prime}\right)\left(\underline{y} \circ \underline{y}^{\prime}\right)
$$

and $\{\underline{N}\}_{S}$, the "scalar of $\stackrel{N}{=}$, is such that

$$
\stackrel{N}{=} \cdot \stackrel{\widetilde{N}}{=}=\{\stackrel{N}{=}\}_{S}^{2}
$$

The wave functions $\psi_{\mathrm{a}}$ and $\psi_{\mathrm{b}}$ were separated in the form

$$
\psi_{a}=F_{e}\left(r, r_{1}, r_{2}\right) r^{-1} R_{e v J}(r) P_{J M}(\theta, \varnothing),
$$


where the $r_{1}$ are the electronic coordinate, $r$ is the nuclear separation, and $\theta$ and $\varnothing$ are angles giving the orientation of the molecule with respect to a fixed coordinate system. Carrying out the integram tion over the electronic coordinate in Eq. C-22, James and Coolidge obtained an expression of the form

$$
\overline{\mathrm{N}}_{\mathrm{X}}=\mathrm{N}(\mathrm{r}) \mathrm{M}(\theta, \phi)
$$

(The scaler $N(r)$ is sometimes referred to as the quadrupole moment, although this term should properiy be reserved for the instantaneous quadrupole moment, $\underline{N}_{0}$ )

Carrying out the integration over $\stackrel{M}{=}(\theta, \varnothing)$, James and Coolidge obtained the selection rule, $\Delta_{\mathrm{J}}= \pm 2$ or 0 with $0 \longrightarrow 0$ forbidden; that is, the matrix elements vanished for $J=0 \longrightarrow J=0$ transitions and for all others except where $\Delta J= \pm 2$ or 0 . Writing

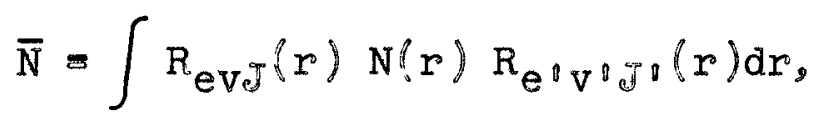

they finaily obtained? their main interest was in the absorption coefficients)

$$
B(b, a)=\frac{4 \pi^{5} y^{2}}{5 c^{3} h^{2}} N^{2} \quad\left\{\begin{array}{l}
\frac{(J+1)(J+2)}{(2 J+1)(2 J+3)}, \Delta J=2 \\
\frac{2 J(J+1)}{3(2 J-I)(2 J+3)}, \Delta J=2 \quad(C-28) \\
\frac{J(J-1)}{(2 J-1)(2 J+1)}, \Delta J=2
\end{array}\right.
$$


To obtain an explicit function for $\mathrm{N}(\mathrm{r})$, James and Coolidge used an electronic wave function of their own device, having found the customary Helteler-London wave function to be inadequate. To a cubic approximation, they obtained

$$
N(r)=e\left\{0.442+0.303(r-1.4) \rightarrow 0.212(r-1.4)^{2}+0.028(r-1.4)^{3}\right\},
$$

where $r$ is in Bohr radi1 $\left(0.529 \times 10^{-8} \mathrm{~cm}\right)$ and $e$ is in units of proton charge. Their analysis was then completed by evaluating $\overline{\mathrm{N}}$. For this purpose, they used vibrational wave functions $R_{\text {evJ }}(r)$ corresponding to the "Posche-Teller" potential for diatomic molecules, which is of the form

$$
V(r)=\operatorname{hcR} \frac{m}{\mu} a^{2}\left[\frac{v(\psi-1)}{\sinh ^{2} a\left(r-r_{0}\right)}-\frac{\mu(\mu+1)}{\cosh ^{2} a\left(r-r_{0}\right)}\right] \text {. }
$$

(This potential plays the same role as the more familiar Morse potential but has an extra adjustable parameter.) By the use of a differential analyzer they obtained $\overline{\mathrm{N}}$ for three particular vibrational transitions.

Letting the subscripts $v, v^{\prime}$ indicate the initial and final vibrational states (from the point of view of an absorption), they found

$$
\left.\begin{array}{l}
\overline{\mathrm{N}}_{0,1}=-0.0441 \\
\overline{\mathrm{N}}_{0,2}=-0.0166 \\
\overline{\mathrm{N}}_{0,3}=-0.0044
\end{array}\right\} \times \quad\left(4.8029 \times 10^{10}\right)\left(0.529 \times 10^{-8}\right)^{2},
$$


where the common factor converts $\bar{N}$ to the proper cgs units for a quadrupole moment, namely, esu- $\mathrm{cm}^{2}$ or $\operatorname{erg} \frac{1}{2}-\mathrm{cm}^{5 / 2}$.

Using Eqs. $C-28, C-31,6-5$, and $6-6, B(a, b)$ and $A(b, a)$ for vibration-rotation transitions between the ground vibrational state and the first three excited vibrational states are easily computed. Table III gives some representative values and the associated wavenumber and wavelength of the transition as given by Herzberg (Ref. 52). The unprimed and primed quantities refer to states $a$ and $b$, respectively。 
Blank page 
REFERENCES

1. Eyring, $H_{0}$, Hirschfelder, J. O., and Taylor, $H_{0}$ S., "The Theoretical Treatment of Chemical Reactions Produced by Ionization Processes, Part I." Journal of Chemlcal Physics $4(1936), 479-491$.

2. Murray, $R_{0} L_{0}$, "Mass-Spectroscopic Study of Ionic Reactions in Hydrogen." Journal of Applied Physics 23 (1952), 6-11.

3. Ortenburger, I. $B_{0}$, Hertzberg, $M_{0}$, and Ogg, R。 A., Jro, "Secondary Reactions in Hydrogen. Journal of Applied Physics $33(1960), 579-583$.

4. Steacie, E。 Wo R。, Atomic and Free Radical Reactions. New York: Reinhold Publishing Corporation (1946), 38。

5. Massey, H. S. Wo, "Excitation and Ionization of Atoms by Electron Impact." Handbuch der Physics XXXVI/1 (1955), 307-339.

6. Pauling, $L_{0}$, and Wilson, $E_{0} B_{0}$, Introduction to Quantum Mechanics. New York: McGraw-H111 Book Co., Inc.(1935), 344, 354.

7. James, H. M。, Coolidge, A. So, and Present, R. Do, "The HeitlerIondon Repulsive State of Hydrogen." Journal of Chemical Physics $4(1936), 187-193$.

8. Rutgers, A. Jo, Physical Chemistry. New York: Interscience Publishers, Inc. (1954), 567.

9. Stranathan, Jo $D_{0}$, The Particles of Modern Physics. Toronto: The Blakiston Company $(1946), 234,336$.

10. Hirschfelder, Jo, Eyring, $H_{0}$, and Rosen, N., "Calculation of Energy of $\mathrm{H}_{3}^{+}$Ion." Journal of Chemical Physics 4 (1936), 130-133.

11. Hirschfelder, Jo, Eyring, $H_{0}$, and Rosen, $N_{0}$, "Calculation of Energy of $\mathrm{H}_{3}$ Molecule." Journal of Chemical Physics 4 (1936), 121-130。

12. Hirschfelder, J., Eyring, $H_{0}$, and Topley, B., "Reactions Involving Hydrogen Molecules and Atoms. Journal of Chemical Physics 4 $(1936), 170-1 \%$

13. Schwinger, J., and Teller, E., "The Scattering of Neutrons by Ortho- and Parahydrogen." Physical Review 52 (1937), 286-295. 


\section{REFERENCES (cont'd )}

14. Bauer, E., "Dissociation of Hydrogen Molecules by Vibrational Excitation and Three-Body Recombination Coefficient." Physical Review 84 (1951), 315-321.

15. Meador, W. E., "Calculation of the Energies of Triatomic Hydrogen Using a Modified Roothaan Procedure." Journal of Chemical Physics 29 (1958), 1339-1345.

16. Hirschfelder, J. $O_{\circ}$, and Wigner, E., "Some Quantum-Mechanical Considerations in the Theory of Reactions Involving an Activation Energy." Journal of Chemical Physics 7 (1939), 616-628.

17. Rosen, No, "Lifetimes of Unstable Molecules." Journal of Chemical Physics 1 (1933), 319-326.

18. Scott, R. B., Cryogenic Eng1neering. New York: D. Van Nostrand Company, Inc., (1959), 290.

19. Bóhm, D., Quantum Theory. New York: Prent1ce-Ha11, Inc. (1952), 400 .

20. Shavitt, I., "A Calculation of the Rates of Ortho-Para Conversions and Isotope Exchanges in Hydrogen." Journal of Chemical Physics 31 (1959), 1359-1367.

21. Nettleton, R. E., "Relaxation Theory of Thermal Conduction in Liquids." The Physics of Fluids 3 (1960), 216-225.

22. Van Kranendonk, J., "Rotational and Vibrational Energy Bands in Solid Hydrogen." Physica 25 (1959), 1080-1094.

23. Sarma, G., "Diffusion des Neutrons Lents par L'Hydrogene Liquide," Inelastic Scattering of Neutrons in Solids and Liquids (Symposium on Inelastic Scattering of Neutrons in Solids and Liquids, Vienna, 1960), Vienna: IAEA (1961), 397.

24. AlIIn, E。 Jo, Feldman, T., and Welsh, Ho I., "Raman Spectra of Liquid and Solid Hydrogen." Journal of Chemical Physics 24 (1956), 1116-1117.

25. Eyring, H., Gershinowitz, H., and Sun, C. E., "The Absolute Rate of Homogeneous Atomic Reactions." Journal of Chemical Physics 3 (19.35), 786-796. 
REFERENCES (cont' $\alpha$ )

26. Griffing, Vo, and Macek, $A_{0}$, "Studies of the Interaction Between Stable Molecules and Atoms. III: A Molecular Orbital Treatment of the Quadratic Form of $\mathrm{H}_{4}{ }^{\text {" }}$ " Journal of Chemical Physies 23 $(1954), 1029-1034$.

27. Griffing, V. and Vanderslice, J. To, "Studies of the Interaction Between Stable Molecules and Atoms. IV: The Energy of the Linear $\mathrm{H}_{4}$ Complex." Journal of Chemical Physics 23 (1954), 1035-1038.

28. Amdur, Io, "The Recombination of Hydrogen Atoms. II: Relative Recombination Rates of Atomic Hydrogen and Atomic Deuterium." Journal of the American Chemlcal Soclety 57 (1935), 856-858.

29. Steiner, W. "The Recombination of Hydrogen Atoms." Transactions of the Faraday Society 31 (1935), 623-636.

30. Mott, No F., and Massey, H. So Wo Theory of Atomic Collisions. Oxford: Clarendon Press (1949), 293.

31. Bergmann, $I_{0}$, UItrasonics. New York: John Wiley and Sons, Inc. $(1938), 143$.

32. Stewart, E. So, "Dispersion of the Velocity and Anamolous Absorption of Sound in Hydrogen." Physical Review 69 (1946), $632-640$ 。

33. Salkoff, M., and Bauer, E., "Excltation of Molecular Vibration on Collision." Journal of Chemical Physics 29 (1958), 26-31.

34. Schiff ${ }^{\circ} I_{0}$ I., Quantum Mechanics. New York: McGraw-H1II Book Company, Inc. $(1955), 304,62,195 \mathrm{ff}$.

35. Morse, $B$ o Mo, "Diatomic Molecules According to the Wave Mechanics. II: Vibrational Leve1s." Physical Review 34 (1929), 57-64.

36. Richards, Wo To, "Supersonic Phenomena." Reviews of Modern Physics 12 (1939), 36-64.

37. Sears, Fo E. Thermodynamics, Cambridge: Addison-Wesley Publishing $\mathrm{CO}_{0}$, Inc. (1953), 289, 325.

38. Zwanzig, R., "Theory of Vibrational Relaxation in Liquids." Journal of Chemical Physics 34 (1960), 1931-1935. 


\section{REF ERENCES (cont'd)}

39. Weinreb, A., "Discrimination Between 'Radiative' and 'NonRadiative' Transfer of Molecular Excitation Energy in Liquid Systems." Journal of Chemical Phys1cs 27 (1957), 133-136.

40. Bourgin, D. G., "IIne Intensities in the Hydrogen-Chloride Fundamental Band." Physical Review 29 (1928), 794-816.

41. Fowler, R. H., Statist1cal Mechanics. Cambridge: The University Press (1955), 696, 17.

42. Condon, E. U., and Shortley, G. $H_{0}$, Theory of Atomic Spectra. Cambridge: The University Press (1957), 82, 84.

43. Van Kranendonk, J., "Theory of Induced Inferared AbsorptIon." Physica 23 (1957), 825-837.

44. Van Kranendonk, J., "Induced Infrared Absorption in Gases. Calculation of the Binary Absorption Coefficients of Symmetrical Diatomic Molecules." Physica 24 (1956), 347-362.

45. Van Kranendonk, J., "Induced Infrared Absorption in Gases. Calculation of the Ternary Absorption Coefficients of Symmetrical Diatomic Molecules." Physica 25 (1959), 337-342.

46. Hareg W. F. J., and Welsh, H. L., "Pressure-Induced Infrared Absorption of Hydrogen and Hydrogen-Foreign Gas Mixtures in the Range 1500-5000 Atmospheres. "Canadian Journal of Physics 36 $(1958), 88-103$.

47. Allin, E. J., Hare, W. F. J., and MacDonald, R. E., "Infrared Absorption of Liquid and Solid Hydrogen." Physlcal Review 98 $(1955), 554-555$.

48. Gush, H. F. et al. "Double Transitions in the Infrared Spectrum of Solid Hydrogen." Physical Review 106 (1957), 1101-1102.

49. Welsh, H. I. et al., "Induced Infrared Absorptions of $\mathrm{H}_{2}$, $\mathrm{N}_{2}$, and 0 in the First overtone Regions." Phys1cal Review 83 (1951), 1264.

50. Lass, H., Vector and Tensor Analysis. New York: McGraw-H1.11 Book Company, Inc. (1950), 175 . 


\section{REFERENCES (cont'd)}

51. James, $H_{\circ} M_{\circ}$, and Coolidge, $A_{\circ} S_{0}$, "Quadrupole RotationVibration Spectrum of $\mathrm{H}_{2}$." The Astrophysical Journal 87 (1938), 438-459.

52. Herzberg, G., "On the Possibility of Detecting Molecular Hydrogen and Nitrogen in Planetary and Stellar Atmospheres by Their Rotation-Vibration Spectra." The Astrophysical Journal 87 (1938), 428-437. 
Blank page 
DIS TR IBUTION

FZK-9-180 31 October 1962

Addressee

ASRCNL

ASR CPR-2

ASRMCE - 1

ASRMPE-2

ASBMPT

ASTEN

ASAPRD-NS

GD/Convair

Convair-ASTRO

TIS

Lockheed-Ga.

Boeing AFPR

Douglas, Dept A26

North American

Pratt and Whitney

ORNL

Naval Ord Lab

Batte IIe-REIC

AIr Univ IIb

Radioplane

Repubilc Avia

Vought-Aero

AFSWC (Tech Inf)

NASA-MSFC

NASA-Washington

AFPR-RCRFE

AFRDC-AE

ASRSMX -2

ASRKIMA

TRG

ASTIA

Sandia

Bureau of Ships

DOFL (Chief-230)

Aerojet-General Nucleonics

IBM

White Sands

Brookhaven

NDA
No. of Copies

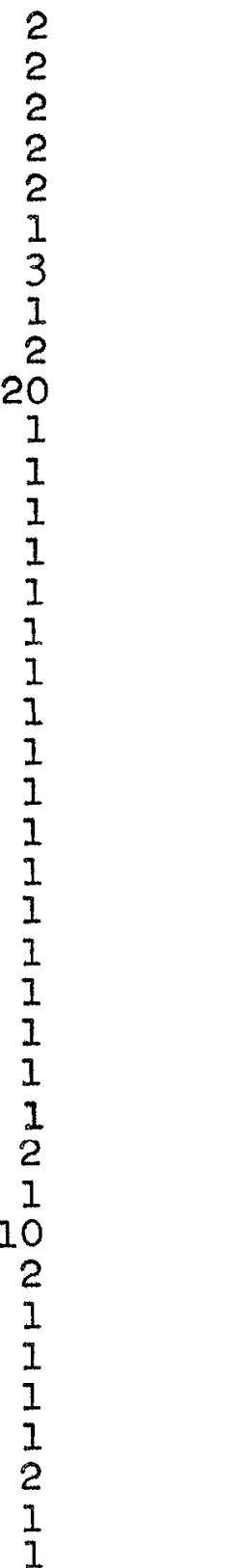




\section{DISTRIBUTION (Cont, $d$ )}

Addressee

US-NADC

AFSWC (SWRB)

Lockheed Missile Div. Minneapolis-HoneyweII

ASRCNE-I

Esso Res

Shell Dev

Cal Res Corp

Defense Atomic Sup Ag

Midwest Res Inst

Hughes

Picatinny Arsenal
No. of Coptes

1

1

1

1

1

1

1

1

1

1

1 